\title{
NON-COMMUTATIVE CREPANT RESOLUTIONS: SCENES FROM CATEGORICAL GEOMETRY
}

\author{
GRAHAM J. LEUSCHKE
}

\begin{abstract}
Non-commutative crepant resolutions are algebraic objects defined by Van den Bergh to realize an equivalence of derived categories in birational geometry. They are motivated by tilting theory, the McKay correspondence, and the minimal model program, and have applications to string theory and representation theory. In this expository article I situate Van den Bergh's definition within these contexts and describe some of the current research in the area.
\end{abstract}

Contents

\begin{tabular}{l|l|} 
Introduction & 1 \\
A. Morita equivalence & 4 \\
B. (Quasi)coherent sheaves & 5 \\
C. Derived categories of modules & 6 \\
D. Derived categories of sheaves & 10 \\
E. Example: Tilting on projective space & 12 \\
F. The non-existence of non-commutative spaces & 16 \\
G. Resolutions of singularities & 21 \\
H. The minimal model program & 22 \\
I. Categorical desingularizations & 27 \\
J. Example: the McKay correspondence & 31 \\
K. Non-commutative crepant resolutions & 31 \\
L. Example: normalization & 34 \\
M. MCM endomorphism rings & 36 \\
N. Global dimension of endomorphism rings & 37 \\
O. Rational singularities & 39 \\
P. Examples: finite representation type & 43 \\
Q. Example: the generic determinant & 45 \\
R. Non-commutative blowups & \\
S. Omissions and open questions & References
\end{tabular}

\section{INTRODUCTION}

A resolution of singularities replaces a singular algebraic variety by a non-singular one that is isomorphic on a dense open set. As such, it is a great boon to the algebraic geometer, allowing the reduction of many calculations and constructions to the case of a smooth variety. To the pure commutative algebraist, however, this process can seem like the end of a story rather than the beginning: it replaces a well-understood thing, the spectrum of a ring, with a much more mysterious thing glued together out of other spaces. Put simply, a resolution of singularities of an affine scheme $\operatorname{Spec} R$ is almost never another affine scheme (but

Date: August 22, 2011.

2010 Mathematics Subject Classification. Primary: 13-02, 14A22, 14E15, 16S38; Secondary: $13 \mathrm{C} 14,13 \mathrm{D} 09$.

Key words and phrases. non-commutative algebraic geometry, categorical geometry, NCCR, noncommutative crepant resolution, McKay correspondence, minimal model program, tilting.

The author was partly supported by NSF grant DMS-0902119. 
see $\S \mathrm{L})$. One cannot in general resolve singularities and stay within the categories familiar to commutative algebraists.

The usual solution, of course, is to expand one's landscape on the geometric side to include more complicated schemes. There are plenty of good reasons to do this other than resolving singularities, and it has worked well for a century. Locally, the more convoluted objects are built out of affine schemes/commutative algebra, so one has not strayed too far.

Here is another alternative: expand the landscape on the algebraic side instead, to include non-commutative rings as well as commutative ones. This suggestion goes by the name "non-commutative algebraic geometry" or, my preference, "categorical geometry". For some thoughts on the terminology, see $\S \mathrm{F}$. Whatever the name, the idea is to treat algebraic objects, usually derived categories, as coming from geometric objects even when no such geometric things exist. Since one trend in algebraic geometry in the last forty years has been to study algebraic varieties indirectly, by studying their (derived) categories of quasicoherent sheaves, one can try to get along without the variety at all. Given a category of interest C, one can postulate a "non-commutative" space $X$ such that the (derived) category of quasicoherent sheaves on $X$ is $C$, and write $C=D^{b}(Q \operatorname{ch} X)$. In this game, the derived category $i s$ the geometry and the symbol $X$ simply stands in as a grammatical placeholder; the mathematical object in play is $C$.

Of course, such linguistic acrobatics can only take you so far. The bonds between algebra and geometry cannot be completely severed: the "non-commutative spaces" must be close enough to the familiar commutative ones to allow information to pass back and forth. This article is about a particular attempt to make this program work.

The idea of non-commutative resolutions of singularities appeared around the same time in physics [BL01, BD02, Ber02, DGM97] and in pure mathematics, notably [BKR01, B002]. In 2002, inspired by Bridgeland's proof [Bri02] of a conjecture due to Bondal and Orlov, Van den Bergh [VdB04b] proposed a definition for a non-commutative crepant resolution of a ring $R$. This is an $R$-algebra $\Lambda$ which is (a) finitely generated as an $R$-module, (b) generically Morita equivalent to $R$, and (c) has finite global dimension. These three attributes are supposed to stand in for the components of the definition of a resolution of singularities: it is (a) proper, (b) birational, and (c) non-singular. The additional "crepancy" condition is a certain symmetry hypothesis on $\Lambda$ which is intended to stand in for the condition that the resolution of singularities not affect the canonical sheaf. See $\$ \mathrm{~K}$ for details.

My main goal for this article is to motivate the definition of a non-commutative crepant resolution (Definition K.4). In order to do that effectively, I will attempt to describe the contexts out of which it arose. These are several, including Morita theory and tilting, the McKay correspondence, the minimal model program of Mori and Reid, and especially work of Bondal and Orlov on derived categories of coherent sheaves. Of course, the best motivation for a new definition is the proof of a new result, and I will indicate where the new concepts have been applied to problems in "commutative" geometry. Finally, the article contains a healthy number of examples, both of existence and of non-existence of non-commutative crepant resolutions. Since it is not at all clear yet that the definitions given below are the last word, we can hope that reasoning by example will point the way forward.

As Miles Reid writes in [Rei00],

It is widely appreciated that mathematicians usually treat history in a curiously dishonest way, rewriting the history of the subject as it should have 
been discovered [....] The essential difficulty seems to be that the story in strictly chronological order will not make sense to anyone; the writer wants to give an explanation based on the logical layout of the subject, whatever violence it does to historical truth.

This article will be guilty of the dishonesty Reid suggests, intentionally in some places and, I fear, unintentionally in others. I do intend to build a certain logical layout around the ideas below, and I sincerely apologize to any who feel that violence has been done to their ideas.

Here is a thumbnail sketch of the contents. The first few sections consider, on both the algebraic side and the geometric, the reconstruction of the underlying ring or space from certain associated categories. The obstructions to this reconstruction-and even to reconstruction of the commutative property-are explained by Morita equivalence $(\S \underline{\mathrm{A}})$ and tilting theory $(\$ \$ \mathrm{C}-\mathrm{D})$. $\$ \mathrm{E}$ contains a central example: Beilinson's "tilting description" of the derived category of coherent sheaves on projective space.

This is not intended to be a comprehensive introduction to non-commutative algebraic geometry; for one thing, I am nowise competent to write such a thing. What I cannot avoid saying is in $\$ \mathrm{~F}$.

The next two sections give synopses of what I need from the geometric theory of resolutions of singularities and the minimal model program, followed in $\$ \mathbb{I}$ by some remarks on purely category-theoretic replacements for resolutions of singularities. Another key example, the McKay correspondence, appears in $\S J$,

At last in $\$ \mathbb{K}$ I define non-commutative crepant resolutions. The definition I give is slightly different from Van den Bergh's original, but agrees with his in the main case of interest. The next few sections $\$ \$ \mathrm{~L}-\mathrm{O}$ focus on particular aspects of the definition, recapping some related research and focusing on obstructions to existence. In particular I give several more examples of existence and non-existence of non-commutative crepant resolutions. Two more families of examples take up $\$ \mathrm{P}$ and $\S \mathrm{Q}$; rings of finite representation type and the generic determinantal hypersurface. Here tilting returns, now as a source of non-commutative crepant resolutions. I investigate a potential theory of "non-commutative blowups" in $\S \Omega$, and give very quick indications of some other examples in $\$ \mathbb{S}$, That section also lists a few open questions and gestures at some topics that were omitted for lack of space, energy, or expertise.

Some results are simplified from their published versions for expository reasons. In particular I focus mostly on local rings, allowing some cleaner statements at the cost of generality, even though such generality is in some cases necessary for the proofs. In any case I give very few proofs, and sketchy ones at that. The only novel contribution is a relatively simple proof, in $\$ \mathrm{Q}$, of the $m=n$ case of the main theorem of [BLV10b].

The reader I have in mind has a good background in commutative algebra, but perhaps less in non-commutative algebra, algebraic geometry, and category theory. Thus I spend more time on trivialities in these latter areas than in the first. I have tried to make the references section comprehensive, though it surely is out of date already.

I am grateful to Jesse Burke, Hailong Dao, Kos Diveris, and Michael Wemyss for insightful comments on earlier drafts, and to Ragnar-Olaf Buchweitz and Michel Van den Bergh for encouragement in this project, as well as for years of enjoyable mathematical interaction. 
Conventions. All modules will be left modules, so for a ring $\Lambda$ I will denote by $\Lambda$-mod the category of finitely generated left $\Lambda$-modules, and by $\bmod -\Lambda=\Lambda^{\mathrm{op}}$-mod the category of finitely generated left $\Lambda^{\mathrm{op}}$-modules. Other categories of modules will be defined on the fly. Capitalized versions of names, namely $\Lambda$-Mod, etc., will denote the same categories without any hypothesis of finite generation.

Throughout $R$ and $S$ will be commutative noetherian rings, usually local, while $\Lambda$ and its Greek-alphabet kin will not necessarily be commutative.

\section{A. Morita equivalence}

To take a representation-theoretic view is to replace the study of a ring by the study of its (abelian category of) modules. Among other advantages, this allows to exploit the tools of homological algebra. A basic question is: How much information do we lose by becoming representation theorists? In other words, when are two rings indistinguishable by their module categories, so that $\Lambda$-mod and $\Gamma$-mod are the same abelian category for different rings $\Lambda$ and $\Gamma$ ?

To fix terminology, recall that a functor $\mathrm{F}: \mathrm{A} \longrightarrow \mathrm{B}$ between abelian categories is fully faithful if it induces an isomorphism on Hom-sets, and dense if it is surjective on objects up to isomorphism. If $F$ is both fully faithful and dense, then it is an equivalence [ML98, IV.4], that is, there is a functor $G: B \longrightarrow A$ such that both compositions are isomorphic to the respective identities. In this case write $A \simeq B$. Equivalences preserve and reflect essentially all "categorical" properties and attributes: mono- and epimorphisms, projectives, injectives, etc. Thus the question above asks when two module categories are equivalent.

Morita's theorem on equivalences of module categories [Mor58, Section 3] completely characterizes the contexts in which $\Lambda$-mod $\simeq \Gamma$-mod for rings $\Lambda$ and $\Gamma$. First I define some of the necessary terms.

Definition A.1. Let $\Lambda$ be a ring and $M \in \Lambda$-Mod.

(i) Denote by add $M$ the full subcategory of $\Lambda$-Mod containing all direct summands of finite direct sums of copies of $M$.

(ii) Say $M$ is a generator (for $\Lambda$-mod) if every finitely generated left $\Lambda$-module is a homomorphic image of a finite direct sum of copies of $M$. Equivalently, $\Lambda \in$ add $M$.

(iii) Say $M$ is a progenerator if $M$ is a finitely generated projective module and a generator. Equivalently, add $\Lambda=\operatorname{add} M$.

Theorem A.2 (Morita equivalence, see e.g. [Gab62, Chap. V]). The following are equivalent for rings $\Lambda$ and $\Gamma$.

(i) There is an equivalence of abelian categories $\Lambda$ - $\bmod \simeq \Gamma$ - $\bmod$.

(ii) There exists a progenerator $P \in \Lambda$-mod such that $\Gamma \cong \operatorname{End}_{\Lambda}(P)^{\mathrm{op}}$.

(iii) There exists a $\left(\Lambda\right.$-Г)-bimodule ${ }_{\Lambda} P_{\Gamma}$ such that the functor $\operatorname{Hom}_{\Lambda}(P,-): \Lambda$-mod $\longrightarrow$ $\Gamma$-mod is an equivalence.

In this case, say that $\Lambda$ and $\Gamma$ are Morita equivalent.

Interesting bits of the history of Morita's theorem, as well as his other work, can be found in [AGHZ97].

An immediate corollary of Morita's theorem will be useful later. 
Corollary A.3. Let $\Lambda$ be a ring and $M, N$ two $\Lambda$-modules such that $\operatorname{add} M=\operatorname{add} N$, equivalently $M$ is a direct summand of $N^{s}$ for some $s$ and $N$ is a direct summand of $M^{t}$ for some $t$. Then $\operatorname{End}_{\Lambda}(M)$ and $\operatorname{End}_{\Lambda}(N)$ are Morita equivalent via the functors $\operatorname{Hom}_{\Lambda}(M, N) \otimes_{\operatorname{End}_{\Lambda}(M)}-$ and $\operatorname{Hom}_{\Lambda}(N, M) \otimes_{\operatorname{End}_{\Lambda}(N)}-$.

Among the consequences of Theorem A.2, the most immediately relevant to our purposes are those related to commutativity. One can show [Lam99, 18.42] that if $\Lambda$ and $\Gamma$ are Morita equivalent, then the centers $Z(\Lambda)$ and $Z(\Gamma)$ are isomorphic. It follows that two commutative rings $R$ and $S$ are Morita equivalent if and only if they are isomorphic. On the other hand, in general Morita equivalence is blind to the commutative property. Indeed, the free module $\Lambda^{n}$ is a progenerator for any $n \geqslant 1$, so that $\Lambda$ and the matrix ring $\operatorname{End}_{\Lambda}\left(\Lambda^{n}\right) \cong \operatorname{Mat}_{n}(\Lambda)$ are Morita equivalent. Even if $\Lambda$ is commutative, $\operatorname{Mat}_{n}(\Lambda)$ will not be for $n \geqslant 2$.

The fact that commutativity is invisible to the module category is a key motivation for categorical geometry. It is interesting to observe that this idea, and even the connection with endomorphism rings, is already present in the Freyd-Mitchell Theorem [Fre64, Theorem 7.34] classifying abelian categories as categories of modules. In detail, the Freyd-Mitchell Theorem says that if $C$ is a category whose objects form a set (as opposed to a proper class) which is closed under all set-indexed direct sums, and $C$ has a progenerator $P$ such that $\operatorname{Hom}_{\mathrm{C}}(P,-)$ commutes with all set-indexed direct sums, then $\mathrm{C} \simeq \Lambda$-Mod for $\Lambda=\operatorname{End}_{\mathrm{C}}(P)$. Different choices of $P$ obviously give potentially non-commutative rings $\Lambda$, even if $C=R$-mod for some commutative ring $R$.

The property of $P$ referred to above will recur later: say that $P$ is $\operatorname{compact}$ if $\operatorname{Hom}_{\mathrm{C}}(P,-)$ commutes with all (set-indexed) direct sums.

The following cousin of Morita equivalence will be essential later on.

Proposition A.4 (Projectivization [ARS97, II.2.1]). Let $\Lambda$ be a ring and $M$ a finitely generated $\Lambda$-module which is a generator. Set $\Gamma=\operatorname{End}_{\Lambda}(M)^{\mathrm{op}}$. Then the functor

$$
\operatorname{Hom}_{\Lambda}(M,-): \Lambda-\bmod \longrightarrow \Gamma-\bmod
$$

is fully faithful, and restricts to an equivalence

$$
\operatorname{Hom}_{\Lambda}(M,-): \operatorname{add} M \longrightarrow \operatorname{add} \Gamma .
$$

In particular, the indecomposable projective $\Gamma$-modules are precisely the modules of the form $\operatorname{Hom}_{\Lambda}(M, N)$ for $N$ an indecomposable module in add $M$.

\section{B. (QUASI)COHERENT SHEAVES}

On the geometric side, it has also long been standard operating procedure to study a variety or scheme $X$ in a representation-theoretic mode by investigating the sheaves on $X$, particularly those of algebraic origin, the quasicoherent sheaves.

Let $X$ be a noetherian scheme. Recall that an $\mathscr{O}_{X}$-module is quasicoherent if it locally can be represented as the cokernel of a homomorphism between direct sums of copies of $\mathscr{O}_{X}$. A quasicoherent sheaf is coherent if those direct sums can be chosen to be finite. Write Qch $X$ for the category of quasicoherent sheaves and $\operatorname{coh} X$ for that of coherent sheaves. Since $X$ is assumed noetherian, these are both abelian categories (for the quasicoherent sheaves, quasi-compact and quasi-separated is enough [BVdB03]).

The category $Q \operatorname{ch} X$ is a natural environment for homological algebra over schemes; for example, computations of cohomology naturally take place in Qch $X$. Now one may ask 
the same question as in the previous section: What information, if any, is lost in passage from the geometric object $X$ to the category $\operatorname{coh} X$ or Qch $X$ ? In this case, the kernel is even smaller: we lose essentially nothing. Indeed, it is not hard to show that for arbitrary complex varieties $X$ and $Y$, the categories $\operatorname{coh} X$ and $\operatorname{coh} Y$ are equivalent if and only if $X$ and $Y$ are isomorphic. The key idea is to associate to a coherent sheaf the closed subset of $X$ on which it is supported; for example, the points of $X$ correspond to the simple objects in coh $X$. See for example [BKS07, Section 8]. More generally, Gabriel [Gab62] taught us how to associate to any abelian category $A$ a geometric realization: a topological space Spec $A$, together with a sheaf of rings $\mathscr{O}_{\mathrm{A}}$. (In fact, the sheaf $\mathscr{O}_{\mathrm{A}}$ is the endomorphism sheaf of the identity functor on A, reminiscent of the Freyd-Mitchell theorem mentioned in the previous section. The space Spec $A$ is nothing but the set of isomorphism classes of indecomposable injective objects of A, with a base for the topology given by $\operatorname{Supp} M=\{[I] \mid$ there is a nonzero arrow $M \longrightarrow I\}$ for noetherian objects $M$.) In the case $\mathrm{A}=\mathrm{Q} \operatorname{ch} X$ for a noetherian scheme $X$, the pair $\left(\operatorname{Spec} \mathrm{A}, \mathscr{O}_{\mathrm{A}}\right)$ is naturally isomorphic to $\left(X, \mathscr{O}_{X}\right)$. This construction has been generalized to arbitrary schemes by Rosenberg [Ros98a, Ros98b], giving the following theorem.

Theorem B.1 (Gabriel-Rosenberg Reconstruction). A scheme $X$ can be reconstructed up to isomorphism from the abelian category Qch X.

Theorem B.1 implies that there is no interesting Morita-type theory for (quasi)coherent sheaves. This is not all that surprising, given that Morita-equivalent commutative rings are necessarily isomorphic. The well-known equivalence between modules over a ring $R$ and quasicoherent sheaves over the affine scheme $\operatorname{Spec} R$ strongly suggests the same sort of uniqueness on the geometric side as on the algebraic.

For projective schemes, Serre's fundamental construction [Ser55] describes the quasicoherent sheaves on $X$ in terms of the graded modules over the homogeneous coordinate ring. Explicitly, let $A$ be a finitely generated graded algebra over a field, and set $X=\operatorname{Proj} A$, the associated projective scheme. Let $\operatorname{GrMod} A, \operatorname{resp} . \operatorname{grmod} A$, denote the category of graded, resp. finitely generated graded, $A$-modules. The graded modules annihilated by $A_{\geqslant n}$ for $n \gg 0$ form a subcategory Tors $A$, resp. $\operatorname{tors} A$, and

$$
\text { Tails } A=\operatorname{GrMod} A / \operatorname{Tors} A \quad \text { and } \quad \operatorname{tails} A=\operatorname{grmod} A / \operatorname{tors} A
$$

are defined to be the quotient categories. This means that two graded modules $M$ and $N$ are isomorphic in Tails $A$ if and only if $M_{\geqslant n} \cong N_{\geqslant n}$ as graded modules for large enough $n$.

Theorem B.2 (Serre). Let A be a commutative graded algebra generated in degree one over $A_{0}=k$, a field, and set $X=\operatorname{Proj} A$. Then the functor $\Gamma_{*}: \operatorname{coh} X \longrightarrow$ tails $A$, defined by sending a coherent sheaf $\mathscr{F}$ to the image in tails $A$ of $\bigoplus_{n=-\infty}^{\infty} H^{0}(X, \mathscr{F}(n))$, defines an equivalence of categories $\operatorname{coh} X \simeq$ tails $A$.

Serre's theorem is the starting point for "non-commutative projective geometry," as we shall see in $\$ \mathrm{~F}$ below. From the point of view of categorical geometry, it is the first instance of a purely algebraic description of the (quasi)coherent sheaves on a space, and thus opens the possibility of "doing geometry" with only a category in hand.

\section{Derived CATEgories of Modules}

Originally introduced as technical tools for organizing homological (or "hyperhomological" [Ver96]) information, derived categories have in the last 30 years been increasingly 
viewed as a basic invariant of a ring or variety. Passing from an abelian category to an associated derived category not only tidies the workspace by incorporating the non-exactness of various natural functors directly into the notation, but in some cases it allows a "truer description" [Bri06] of the underlying algebra or geometry than the abelian category does. For example, there are varieties with non-trivial derived auto-equivalences $\mathrm{D}^{b}(X) \simeq \mathrm{D}^{b}(X)$ that do not arise from automorphisms; one might think of these as additional symmetries that were invisible from the geometric point of view. Another example is Kontsevich's Homological Mirror Symmetry conjecture [Kon95], which proposes an equivalence of certain derived categories related to "mirror pairs" of Calabi-Yau manifolds.

Let us fix some notation. Let $A$ be an abelian category. The homotopy category $\mathrm{K}(\mathrm{A})$ has for objects the complexes over $A$, and for morphisms homotopy-equivalence classes of chain maps. The derived category $D(A)$ is obtained by formally inverting those morphisms in $K(A)$ which induce isomorphisms on cohomology, i.e. the quasi-isomorphisms.

We decorate $K(A)$ and $D(A)$ in various ways to denote full subcategories. For the moment I need only $\mathrm{K}^{b}(\mathrm{~A})$, the full subcategory composed of complexes $C$ having only finitely many non-zero components, and $D^{b}(A)$, the corresponding bounded derived category of $A$.

The homotopy category $K(A)$, the derived category $D(A)$ and their kin are no longer abelian categories, but they have a triangulated structure, consisting of a shift functor (-)[1] shifting a complex one step against its differential and changing the sign of that differential, and a collection of distinguished triangles taking the place occupied by the short exact sequences in abelian categories. A functor between triangulated categories is said to be a triangulated functor if it preserves distinguished triangles and intertwines the shift operators.

Homomorphisms $\varphi: M \longrightarrow N$ in $\mathrm{D}(A)$ are diagrams $M \stackrel{f}{\longleftarrow} P \stackrel{g}{\longrightarrow} N$ of homotopy classes of chain maps, where $f: P \longrightarrow M$ is a quasi-isomorphism and we think of $\varphi$ as $f^{-1} g$. Much more usefully,

$$
\operatorname{Hom}_{\mathrm{D}(A)}(M, N[i])=\operatorname{Ext}_{A}^{i}(M, N)
$$

for all $i \in \mathbb{Z}$ and all $M, N$ in $\mathrm{D}(A)$.

Let us say that two rings $\Lambda$ and $\Gamma$ are derived equivalent if there is an equivalence of triangulated categories $\mathrm{D}^{b}(\Lambda$-Mod $) \longrightarrow \mathrm{D}^{b}(\Gamma-\mathrm{Mod})$.

It is nearly obvious that a Morita equivalence between rings $\Lambda$ and $\Gamma$ gives rise to a derived equivalence $D^{b}(\Lambda-$ Mod $) \simeq D^{b}(\Gamma$-Mod). (Any equivalence between abelian categories preserves short exact sequences.) In general, derived equivalence is a much weaker notion. It does, however, preserve some essential structural information. For example, if $\Lambda$ and $\Gamma$ are derived equivalent, then their Grothendieck groups $K_{0}(\Lambda)$ and $K_{0}(\Gamma)$ are isomorphic [Ric89, Prop. 9.3], as are the Hochschild homology and cohomology groups [Ric91] and the cyclic cohomologies [Hap89]. If $\Lambda$ and $\Gamma$ are derived-equivalent finite-dimensional algebras over a field $k$, then they have the same number of simple modules, and simultaneously have finite global dimension [Hap87, Hap88].

Most importantly for this article, derived-equivalent rings have isomorphic centers [Ric89, Prop. 9.2]. In particular, if $R$ and $S$ are commutative rings, then $\mathrm{D}^{b}\left(R\right.$-Mod) $\simeq \mathrm{D}^{b}(S-\mathrm{Mod})$ if and only if $R \cong S$. Thus there is at most one commutative ring in any derived-equivalence class, another sign that one should look at non-commutative rings for non-trivial derived equivalences. 
All these facts follow from Rickard's Morita theory of derived equivalences, in which the progenerator of Theorem A.2 is replaced by a tilting object. Here is the main result of Rickard's theory.

Theorem C.1 (Rickard [Ric89]). Let $\Lambda$ and $\Gamma$ be rings. The following conditions are equivalent.

(i) $\mathrm{D}^{b}(\Lambda-\mathrm{Mod})$ and $\mathrm{D}^{b}(\Gamma-\mathrm{Mod})$ are equivalent as triangulated categories.

(ii) $\mathrm{K}^{b}(\operatorname{add} \Lambda)$ and $\mathrm{K}^{b}(\operatorname{add} \Gamma)$ are equivalent as triangulated categories.

(iii) There is an object $T \in \mathrm{K}^{b}$ (add $\Lambda$ ) satisfying

(a) $\operatorname{Ext}_{\Lambda}^{i}(T, T)=0$ for all $i>0$, and

(b) add $T$ generates $\mathrm{K}^{b}$ (add $\Lambda$ ) as a triangulated category, such that $\Gamma \cong \operatorname{End}_{\Lambda}(T)$.

If $\Lambda$ and $\Gamma$ are finite dimensional algebras over a field, then these are all equivalent to $\mathrm{D}^{b}(\Lambda$-mod $) \simeq \mathrm{D}^{b}(\Gamma-\bmod )$

A complex $T$ as in condition (iii) is called a tilting complex for $\Lambda$, and $\Gamma$ is tilted from $\Lambda$. Tilting complexes appeared first in the form of tilting modules, as part of Brenner and Butler's [BB80] study of the reflection functors of Bernštein, Gel'fand, and Ponomarev [BGP73]. (The word was chosen to illustrate their effect on the vectors in a root system, namely a change of basis that tilts the axes relative to the positive roots.) Their properties were generalized, formalized, and investigated subsequently by Happel and Ringel [HR82], Bongartz [Bon81], Cline-Parshall-Scott [CPS86], Miyashita [Miy86], and others. Happel seems to have been the first to realize [Hap88] that if $T$ is a $\Lambda$-module of finite projective dimension, having no higher self-extensions $\operatorname{Ext}_{\Lambda}^{>0}(T, T)=0$, and $\Lambda$ has a finite co-resolution $0 \longrightarrow \Lambda \longrightarrow$ $T_{1} \longrightarrow \cdots \longrightarrow T_{r} \longrightarrow 0$ with each $T_{i} \in$ add $T$, then the functor $\operatorname{Hom}_{\Lambda}(T,-): \mathrm{D}^{b}(\Lambda$-Mod $) \longrightarrow$ $\mathrm{D}^{b}\left(\operatorname{End}_{\Lambda}(T)\right.$-Mod) is an equivalence. In their earliest incarnation, tilting modules were defined to have projective dimension one, but this more general version has become standard.

Morita equivalence is a special case of tilting. Indeed, any progenerator is a tilting module. However, see $\$ \mathrm{E}$ below for a pair of derived-equivalent algebras which are not Morita equivalent.

\section{Derived CATEgories of Sheaves}

The first real triumphs of the derived category came in the geometric arena: Grothendieck and coauthors' construction of a global intersection theory and the theorem of RiemannRoch [BGI71] are the standard examples [Căl05]. The idea of the (bounded) derived category of a scheme as a geometric invariant first emerged around 1980 in the work of Beilinson, Mukai, and others. I will describe some of Beilinson's observations in the next §. Mukai found the first example of non-isomorphic varieties which are derived equivalent [Muk81]; he showed that an abelian variety $X$ and its dual $X^{\vee}$ always have equivalent derived categories of quasicoherent sheaves. His construction is modeled on a Fourier transform and is now called a Fourier-Mukai transform [Huy06, HVdB07]. It would draw us too far afield from our subject to discuss Fourier-Mukai transforms in any depth here. Several examples will appear later in the text: see the end of $\$ \mathrm{E}$ and Theorems H.4, J.7, and J.8, It is an important result of Orlov [Orl97] that any equivalence $\mathrm{D}^{b}(\operatorname{coh} X) \longrightarrow \mathrm{D}^{b}(\operatorname{coh} Y)$, for $X$ and $Y$ connected smooth projective varieties, is given by a Fourier-Mukai transform. 
The existence of non-trivial derived equivalences for categories of sheaves means that one cannot hope for a general reconstruction theorem, even for smooth varieties. However, under an assumption on the canonical sheaf $\omega_{X}$, the variety $X$ can be reconstructed from its derived category. Recall that for $X$ a smooth complex variety over $\mathbb{C}, \omega_{X}=\wedge^{\operatorname{dim} X} \Omega_{X / \mathbb{C}}$ is the sheaf of top differential forms on $X$ where $\Omega_{X / \mathbb{C}}$ is the cotangent bundle, a.k.a. the sheaf of 1-forms on $X$. It is an invertible sheaf. Recall further [Har77, II.7] that an invertible sheaf $\mathscr{L}$ is ample if for every coherent sheaf $\mathscr{F}, \mathscr{F} \otimes \mathscr{L}^{n}$ is generated by global sections for $n \gg 0$.

Theorem D.1 (Bondal-Orlov [BO01]). Let $X$ and $Y$ be smooth connected projective varieties over $\mathbb{C}$. Assume that either the canonical sheaf $\omega_{X}$ or the anticanonical sheaf $\omega_{X}^{-1}$ is ample. If $\mathrm{D}^{b}(\operatorname{coh} X) \simeq \mathrm{D}^{b}(\operatorname{coh} Y)$, then $X$ is isomorphic to $Y$.

Note that the result is definitely false for abelian varieties by the result of Mukai mentioned above; in this case $\omega_{X} \cong \mathscr{O}_{X}$ is trivial, so not ample. Calabi-Yau varieties are another example where $\omega_{X} \cong \mathscr{O}_{X}$ is not ample, and the conclusion does not hold.

One consequence of this theorem is that, under the same hypotheses, the group of autoequivalences $\mathrm{D}^{b}(\operatorname{coh} X) \stackrel{\simeq}{\longrightarrow} \mathrm{D}^{b}(\operatorname{coh} X)$ of $X$ is generated by the obvious suspects: Aut $(X)$, the shift (-)[1], and the tensor products $-\otimes_{\mathscr{O}_{X}} \mathscr{L}$ with fixed line bundles $\mathscr{L}$.

Triangulated categories arising in nature like $\mathrm{D}^{b}(\operatorname{coh} X)$ generally have a lot of additional structure: there is a tensor (symmetric monoidal) structure induced from the derived tensor product, among other things. Taking this into account gives stronger results. To give an example, recall that a perfect complex on a scheme $X$ is one which locally is isomorphic in the derived category to a bounded complex of locally free sheaves of finite rank. Perfect complexes form a subcategory $\mathrm{D}^{\text {perf }}(\mathrm{Qch} X)$. As long as $X$ is quasi-compact and separated (noetherian is enough), $\mathrm{D}^{\text {perf }}(\mathrm{Q} c \mathrm{X} X)$ contains precisely the compact objects of $\mathrm{D}(\mathrm{Q} c \mathrm{X} X)$, that is, the complexes $C$ such that $\operatorname{Hom}_{\mathscr{O}_{X}}(C,-)$ commutes with set-indexed direct sums. See [BVdB03, 3.11]. Balmer [Bal02, Bal05] shows that a noetherian scheme $X$ can be reconstructed up to isomorphism from $\mathrm{D}^{\text {perf }}(\mathrm{Qch} X)$, as long as the natural tensor structure is taken into account, and that two reduced noetherian schemes $X$ and $X^{\prime}$ are isomorphic if and only if $D^{\text {perf }}(Q \operatorname{ch} X)$ and $D^{\text {perf }}\left(Q c h X^{\prime}\right)$ are equivalent as tensor triangulated categories.

The theory of tilting sketched in the previous $\S$ has a geometric incarnation as well, which signals the first appearance of non-commutative rings on the geometric side of our story.

Definition D.2. Let $X$ be a noetherian scheme and $T$ an object of $\mathrm{D}(\mathrm{Q} \operatorname{ch} X)$. Say that $T$ is a tilting object if it is compact, is a classical generator for $\mathrm{D}^{\text {perf }}(\mathrm{Qch} X)$, and has no non-trivial self-extensions. Explicitly, this is to say:

(i) $T$ is a perfect complex;

(ii) The smallest triangulated subcategory of $\mathrm{D}(\mathrm{Qch} X)$ containing $T$ and closed under direct summands is $\mathrm{D}^{\text {perf }}(\mathrm{Q} \operatorname{ch} X)$; and

(iii) $\operatorname{Ext}_{\mathscr{O}_{X}}^{i}(T, T)=0$ for $i>0$.

If $T$ is quasi-isomorphic to a complex consisting of a locally free sheaf in a single degree, it is sometimes called a tilting bundle.

The generating condition (ii) is sometimes replaced by the requirement that $T$ generates $\mathrm{D}(\mathrm{Qch} X)$, i.e. that if an object $N$ in $\mathrm{D}(\mathrm{Q} c h X)$ satisfies $\operatorname{Ext}_{\mathscr{O}_{X}}^{i}(T, N)=0$ for all $i \in \mathbb{Z}$, then $N=0$. If an object $T$ classically generates $\mathrm{D}^{\text {perf }}(\mathrm{Qch} X)$ as in the definition, then it generates 
$\mathrm{D}(\mathrm{Q}$ ch $X)$; the converse holds in the presence of the assumption (i) that $T$ is compact. This is a theorem due to Ravenel and Neeman [BVdB03, Theorem 2.1.2].

A class of schemes particularly well-suited for geometric tilting theory consists of those which are projective over a scheme $Z$, which in turn is affine of finite type over an algebraically closed field $k$. This generality allows a wide range of interesting examples, but also ensures, by [Gro61, Théorème 2.4.1(i)], that if $T$ is a tilting object on $X$ then the endomorphism ring $\Lambda=\operatorname{End}_{\mathscr{O}_{X}}(T)$ is a finitely generated algebra over the field $k$. In particular, $\Lambda$ is finitely generated as a module over its center.

The next result is fundamental for everything that follows. It has origins in the work of Beĭlinson presented in the next $\S$, with further refinements in [Bon89, Bae88, BVdB03].

Theorem D.3 (Geometric Tilting Theory [HVdB07, 7.6]). Let $X$ be a scheme, projective over a finite-type affine scheme over an algebraically closed field $k$. Let $T$ be a tilting object in $\mathrm{D}(\mathrm{Qch} X)$, and set $\Lambda=\operatorname{End}_{\mathscr{O}_{X}}(T)$. Then

(i) $\mathbf{R H o m}_{\mathscr{O}_{X}}(T,-)$ induces an equivalence of triangulated categories between $\mathrm{D}(\mathrm{Q} c \mathrm{X} X)$ and $\mathrm{D}\left(\Lambda\right.$-Mod), with inverse $-\stackrel{\mathbf{L}}{\otimes}_{\Lambda} T$.

(ii) If $T$ is in $\mathrm{D}^{b}(\operatorname{coh} X)$, then this equivalence restricts to give an equivalence between $\mathrm{D}^{b}(\operatorname{coh} X)$ and $\mathrm{D}^{b}(\Lambda$-mod $)$.

(iii) If $X$ is smooth, then $\Lambda$ has finite global dimension.

It is not at all clear from this result when tilting objects exist, though it does impose some necessary conditions on $X$. For example, assume that in addition $X$ is projective over $k$ and $T$ is a tilting object in $\mathrm{D}(\mathrm{Q} \operatorname{ch} X)$. Then $\Lambda=\operatorname{End}_{\mathscr{O}_{X}}(T)$ is a finite-dimensional algebra over $k$. The Grothendieck group $K_{0}(\Lambda)$ is thus a free abelian group of finite rank, equal to the number of simple $\Lambda$-modules. This implies that $K_{0}(X)$ is free abelian as well. Thus any torsion in $K_{0}$ rules out the existence of a tilting object.

\section{E. EXAMPLE: TILTing ON PROJECTIVE SPACE}

In this $\S$ I illustrate Theorem D.3 via Beĭlinson's tilting description of the derived category of projective space. The techniques have been refined and are now standard; they have been used, most notably by Kapranov, to construct explicit descriptions of the derived category of coherent sheaves on several classes of varieties. For example, there are tilting bundles on smooth projective quadrics [Kap86], on Grassmannians [Kap84, BLV10a], on flag manifolds [Kap88], on various toric varieties [Kin97, HP06, $\mathrm{HP}]$, and on weighted projective spaces [GL87, Bae88]. Here we stick to projective space.

Let $k$ be a field, $V$ a $k$-vector space of dimension $n \geqslant 2$, and $\mathbb{P}=\mathbb{P}^{n-1}=\mathbb{P}(V)$ the projective space on $V$. We consider two families of $n$ locally free sheaves on $\mathbb{P}$. First let

$$
\mathscr{E}_{1}=\{\mathscr{O}, \mathscr{O}(-1), \ldots, \mathscr{O}(-n+1)\}
$$

where $\mathscr{O}=\mathscr{O}_{\mathbb{P}}$ is the structure sheaf. Also let $\Omega=\Omega_{\mathbb{P}}$ be the cotangent sheaf, so that $\Omega^{i}=\bigwedge^{i} \Omega$ is the $\mathscr{O}$-module of differential $i$-forms on $\mathbb{P}$, and set

$$
\mathscr{E}_{2}=\left\{\Omega^{0}(1)=\mathscr{O}(1), \Omega^{1}(2), \ldots, \Omega^{n-1}(n)\right\} .
$$

Let $T_{1}$ and $T_{2}$ be the corresponding direct sums,

$$
T_{1}=\bigoplus_{a=0}^{n-1} \mathscr{O}(-a) \quad \text { and } \quad T_{2}=\bigoplus_{a=1}^{n} \Omega^{a-1}(a) .
$$


The constituent sheaves of $\mathscr{E}_{1}$ and $\mathscr{E}_{2}$ are related by the tautological Koszul complex on $\mathbb{P}$. Indeed, the Euler derivation $e: V \otimes_{k} \mathscr{O}(-1) \longrightarrow \mathscr{O}$, which corresponds to the identity on $V$ under $\operatorname{Hom}_{\mathscr{O}}\left(V \otimes_{k} \mathscr{O}(-1), \mathscr{O}\right) \cong \operatorname{Hom}_{\mathscr{O}}\left(V \otimes_{k} \mathscr{O}, \mathscr{O}(1)\right) \cong \operatorname{Hom}_{k}(V, V)$, gives rise to a complex

$$
0 \longrightarrow \wedge^{n} V \otimes_{k} \mathscr{O}(-n) \longrightarrow \cdots \longrightarrow \wedge^{1} V \otimes_{k} \mathscr{O}(-1) \longrightarrow \mathscr{O} \longrightarrow 0
$$

on $\mathbb{P}$. In fact it is acyclic [Eis95, Ex. 17.20], and the cokernels are exactly the sheaves $\Omega^{i}$, which decompose the Koszul complex into short exact sequences

$$
0 \longrightarrow \Omega^{a} \longrightarrow \bigwedge^{a} V \otimes_{k} \mathscr{O}(-a) \longrightarrow \Omega^{a-1} \longrightarrow 0 .
$$

Together with the well-known calculation of the cohomologies of the sheaves $\mathscr{O}(-a)[\operatorname{Har} 77$, III.5.1], the identification ${\mathscr{H} o m_{\mathscr{O}}}(\mathscr{O}(-a), \mathscr{O}(-b))=\mathscr{O}(a-b)$, and the fact that $\operatorname{Ext}_{\mathscr{O}}^{i}(-,-)=$ $H^{i}\left({\left.\mathcal{H} O m_{\mathscr{O}}(-,-)\right)}\right.$ on vector bundles, this produces the following data. (See [BLV10b] for a jazzed-up version which holds over any base ring $k$.)

Lemma E.1. Keep the notation established so far in this section.

(i) We have $\operatorname{Ext}_{\mathscr{O}}^{i}(\mathscr{O}(-a), \mathscr{O}(-b))=0$ for all $i>0$, and

$$
\operatorname{Hom}_{\mathscr{O}}(\mathscr{O}(-a), \mathscr{O}(-b)) \cong \operatorname{Sym}_{a-b}(V)
$$

for $0 \leqslant a, b \leqslant n-1$.

(ii) We have $\operatorname{Ext}_{\mathscr{O}}^{i}\left(\Omega^{a-1}(a), \Omega^{b-1}(b)\right)=0$ for all $i>0$, and

$$
\operatorname{Hom}_{\mathscr{O}}\left(\Omega^{a-1}(a), \Omega^{b-1}(b)\right) \cong \bigwedge^{a-b}\left(V^{*}\right)
$$

for $1 \leqslant a, b \leqslant n$, where $V^{*}$ is the dual of $V$.

The lemma in particular implies that the endomorphism rings of $T_{1}$ and $T_{2}$,

$$
\Lambda_{1}=\operatorname{End}_{\mathscr{O}}\left(\bigoplus_{a=0}^{n-1} \mathscr{O}(-a)\right) \cong \bigoplus_{a, b=0}^{n-1} \operatorname{Sym}_{a-b}(V)
$$

and

$$
\Lambda_{2}=\operatorname{End}_{\mathscr{O}}\left(\bigoplus_{a=1}^{n} \Omega^{a-1}(a)\right) \cong \bigoplus_{a, b=0}^{n-1} \Lambda^{a-b}\left(V^{*}\right)
$$

are "spread out" versions of the truncated symmetric and exterior algebras, respectively. This can be made more precise by viewing $\Lambda_{1}$ and $\Lambda_{2}$ as quiver algebras. Consider a quiver on $n$ vertices labeled, say, $0,1, \ldots, n-1$, and having $n$ arrows from each vertex to its successor, corresponding to a basis of $V$, resp. of $V^{*}$. Introduce quadratic relations $v_{i} v_{j}=v_{j} v_{i}$ corresponding to the kernel of the natural map $V \otimes_{k} V \longrightarrow \operatorname{Sym}_{2}(V)$, respectively $v_{i} v_{j}=-v_{j} v_{i}$ corresponding to the kernel of $V^{*} \otimes_{k} V^{*} \longrightarrow \wedge^{2}\left(V^{*}\right)$. The resulting path algebras with relations are isomorphic to $\Lambda_{1}$ and $\Lambda_{2}$, respectively. In [BLV10b] we call these "quiverized" symmetric and exterior algebras.

I have not yet proven that $\Lambda_{1}$ and $\Lambda_{2}$ are derived equivalent to $\mathbb{P}$. For this, it remains to show that the collections $\mathscr{E}_{1}$ and $\mathscr{E}_{2}$ generate the derived category $\mathrm{D}^{b}(\operatorname{coh} \mathbb{P})$. This is accomplished via Beillinson's "resolution of the diagonal" argument. Let $\Delta \subset \mathbb{P} \times \mathbb{P}$ denote the diagonal, and $p_{1}, p_{2}: \mathbb{P} \times \mathbb{P} \longrightarrow \mathbb{P}$ the projections onto the factors. For sheaves $\mathscr{F}$ and $\mathscr{G}$ on $\mathbb{P}$, set

$$
\mathscr{F} \otimes \mathscr{G}=p_{1}^{*} \mathscr{F} \otimes_{\mathbb{P} \times \mathbb{P}} p_{2}^{*} \mathscr{G},
$$


a sheaf on $\mathbb{P} \times \mathbb{P}$. One can show that the structure sheaf of the diagonal $\mathscr{O}_{\Delta}$ is resolved over $\mathscr{O}_{\mathbb{P} \times \mathbb{P}}$ by a Koszul-type resolution

$$
0 \longrightarrow \mathscr{O}(-n) \otimes \Omega^{n}(n) \longrightarrow \cdots \longrightarrow \mathscr{O}(-1) \otimes \Omega^{1}(1) \longrightarrow \mathscr{O}_{\mathbb{P} \times \mathbb{P}} \longrightarrow \mathscr{O}_{\Delta} \longrightarrow 0 .
$$

In particular, $\mathscr{O}_{\Delta}$ is in the triangulated subcategory of $D^{b}(\operatorname{coh}(\mathbb{P} \times \mathbb{P}))$ generated by sheaves of the form $\mathscr{O}(-i) \otimes Y$ for $Y$ in $D^{b}(\operatorname{coh} \mathbb{P})$. The same goes for any object of the form $\mathscr{O}_{\Delta} \nabla \mathbf{L} p_{1}^{*} X$ with $X$ in $\mathrm{D}^{b}(\operatorname{coh} \mathbb{P})$ as well. Push down now by $p_{2}$ and use the projection formula to see that $X=\mathbf{R} p_{2 *}\left(\mathscr{O}_{\Delta} \stackrel{\mathbf{L}}{\otimes} \mathbf{L} p_{1}^{*} X\right)$ belongs to the triangulated subcategory of $\mathrm{D}^{b}(\mathrm{coh} \mathbb{P})$ generated by $\mathscr{O}(-i) \otimes \mathbf{R} p_{2 *} p_{1}^{*} Y$. The factor $\mathbf{R} p_{2 *} p_{1}^{*} Y$ is represented by the complex of $k$ vector spaces with zero differential $\mathbf{R} \Gamma(Y)$, and hence $\mathscr{E}_{1}=\{\mathscr{O}, \mathscr{O}(-1), \ldots, \mathscr{O}(-n+1)\}$ generates $\mathrm{D}^{b}(\operatorname{coh} \mathbb{P})$. On the other hand, reversing the roles of $p_{1}$ and $p_{2}$ gives the result for $\mathscr{E}_{2}=\left\{\mathscr{O}(1), \Omega^{1}(2), \ldots, \Omega^{n-1}(n)\right\}$ as well.

This discussion proves the following theorem.

Theorem E.2 (Beĭlinson). Let $k$ be a field, $V$ a vector space of dimension $n \geqslant 2$ over $k$, and $\mathbb{P}=\mathbb{P}(V)$. The vector bundles

$$
T_{1}=\bigoplus_{a=0}^{n-1} \mathscr{O}_{\mathbb{P}}(-a) \quad \text { and } \quad T_{2}=\bigoplus_{a=1}^{n} \Omega_{\mathbb{P}}^{a-1}(a)
$$

are tilting bundles on $\mathbb{P}$. In particular, there are equivalences of triangulated categories

$$
\mathrm{D}^{b}\left(\Lambda_{1} \text {-mod }\right) \simeq \mathrm{D}^{b}(\operatorname{coh} \mathbb{P}) \simeq \mathrm{D}^{b}\left(\Lambda_{2} \text {-mod }\right)
$$

defined by $\mathbf{R H o m}_{\mathscr{O}_{\mathbb{P}}}\left(T_{i},-\right)$ for $i=1,2$, where $\Lambda_{i}=\operatorname{End}_{\mathscr{O}_{\mathbb{P}}}\left(T_{i}\right)$.

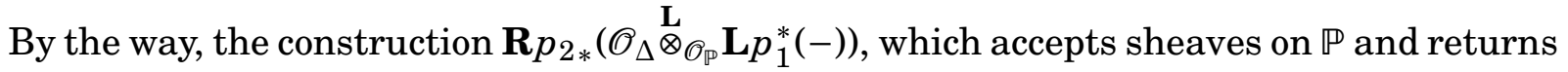
sheaves on $\mathbb{P}$, is an example of a Fourier-Mukai transform, the definition of which was gracefully avoided in $\S \mathbb{D}$. Replacing $\mathscr{O}_{\Delta}$ by any other fixed complex in $D^{b}(\operatorname{coh}(\mathbb{P} \times \mathbb{P}))$ would give another.

\section{F. THE NON-EXISTENCE OF NON-COMMUTATIVE SPACES}

As mentioned in the Introduction, I personally am reluctant to use the phrase "noncommutative algebraic geometry" to describe results like Beullinson's in $\S \mathrm{E}$. While the phrase is certainly apposite on a word-by-word basis, given that the ideas are a natural blend of algebraic geometry and non-commutative algebra, I find that using it in public leads immediately to being asked awkward questions like, "What on earth is non-commutative geometry?" While many people have offered thoughtful, informed answers to this question[Smi, Kee03, Kal09, Kal08, Mor08, VW97, Lau03, Jør, SVdB01, Gin05] are some of my personal favorites-I find the whole conversation distracting from the more concrete problems at hand. I propose instead that results like Berlinson's and those to follow in later sections should be considered as part of "categorical geometry". The name seems unclaimed, apart from an online book from 1998.

In this $\S$ I say a few words about a couple of approaches to building a field called noncommutative algebraic geometry. I have chosen a deliberately provocative title for the section, so that there can be no question that these are opinionated comments. The reader who is intrigued by the ideas mentioned here would do well to seek out a less idiosyncratic, more comprehensive introduction such as those cited in the previous paragraph. 
One potential pitfall for the prospective student of non-commutative geometry is that there are several disparate approaches. For one thing, the approach of Connes and his collaborators [JM97], which some hope will explain aspects of the Standard Model of particle physics or even prove the Riemann Hypothesis, is based on differential geometry and $C^{*}$ algebras, and is, as far as I can tell, completely separate from most of the considerations in this article. More subtly, even within non-commutative algebraic geometry, there are a few different points of view. I do not consider myself competent even to give references, for fear of giving offense by omission.

So what is the problem here? Why can't one simply do algebraic geometry, say at the level of [Har77], over non-commutative rings [MOc]? There have been several sustained attempts to do exactly this, starting in the 1970s. There are a couple of immediate obstacles to a naïve approach.

The first problem is to mimic the fact that a ring $R$ can be recovered from the Zariski topology on the prime spectrum $\operatorname{Spec} R$ and the structure sheaf $\mathscr{O}_{\mathrm{Spec} R}$. (One finds, of course, $H^{0}\left(\operatorname{Spec} R, \mathscr{O}_{\operatorname{Spec} R}\right)=R$.) Both of these sets of information depend essentially on localization. For non-commutative rings, the prime spectrum is rather impoverished; for example, the Weyl algebra $\mathbb{C}\langle x, y\rangle /(y x-x y-1)$ has trivial two-sided prime spectrum. Even ignoring this difficulty, localization for non-commutative rings [Ran06, Jat86] only functions well for Øre sets, and the complement of a prime ideal need not be an Øre set.

One possible resolution of the problem would be to focus on the quotient modules $\Lambda / \mathfrak{p}$ instead of the prime ideals $\mathfrak{p}$. The points of a commutative affine variety $X$ (over $\mathbb{C}$, say) are in one-one correspondence with the simple modules over the coordinate ring $\mathbb{C}[X]$. Furthermore, a point $x \in X$ is a non-singular point if and only if the corresponding simple module $\mathbb{C}[X] / \mathfrak{m}_{x}$ has finite projective dimension.

Unfortunately, here there is a second problem: finite projective dimension, even finite global dimension, is a very weak property for non-commutative rings. For example, there is no Auslander-Buchsbaum Theorem giving a uniform upper bound on finite projective dimensions over a given ring; the existence of such a bound over an Artin algebra is called the finitistic dimension conjecture, and has been open since at least 1960 [Bas60, HZ95]. There are a host of additional technical problems to be overcome. It's unknown, for instance, whether finite global dimension implies primeness (as regularity implies domain for a commutative local ring); the Jacobson radical might fail to satisfy the Artin-Rees property [BHM82], derailing the standard proof. Pathologies abound: for example, there is a local noetherian domain $\Lambda$ of global dimension 3 such that every quotient ring other than $\Lambda$ itself, 0, and $\Lambda / \operatorname{rad}(\Lambda)$ has infinite global dimension [BHM82, Example 7.3].

Restricting to a smaller class of rings solves some of these problems. For example, the class of rings $\Lambda$ which are finitely generated modules over their center $Z(\Lambda)$ are much betterbehaved than the norm. For example, $\Lambda$ is left and right noetherian if $Z(\Lambda)$ is, so that $\operatorname{gldim} \Lambda=\operatorname{gldim} \Lambda^{\mathrm{op}}$. The "lying over", "incomparability", and "going up" properties hold for prime ideals along the extension $Z(\Lambda) \hookrightarrow \Lambda$ [Pas89, Theorem 16.9]. Furthermore, the following reassuring results hold [IR08, Section 2].

Proposition F.1. Let $(R, \mathfrak{m})$ be a local ring and $\Lambda$ a module-finite $R$-algebra. Let $M$ be a finitely generated $\Lambda$-module.

(i) The dimension of $M$, defined by $\operatorname{dim} M=\operatorname{dim}\left(R / \operatorname{Ann}_{R}(M)\right)$, is independent of the choice of central subring $R$ over which $\Lambda$ is a finitely generated module. 
(ii) The depth of $M$, defined by $\operatorname{depth} M=\inf \left\{i \mid \operatorname{Ext}_{R}^{i}(R / \mathfrak{m}, M) \neq 0\right\}$, is also independent of the choice of $R$.

(iii) (Ramras [Ram69]) We have

$$
\operatorname{depth} M \leqslant \operatorname{dim} M \leqslant \operatorname{injdim}_{\Lambda} M .
$$

In particular, if $\Lambda$ is a torsion-free $R$-module and gldim $\Lambda<\infty$, then $\operatorname{inj}_{\operatorname{dim}} \Lambda=$ gldim $\Lambda$ [Aus86a, Lemma 1.3], so that

$$
\operatorname{depth}_{R} \Lambda \leqslant \operatorname{dim} R \leqslant \operatorname{gldim} \Lambda .
$$

(iv) ([Rai87] or [Goo89]) The global dimension of $\Lambda$ is the supremum of $\operatorname{pd}_{\Lambda} L$ over all $\Lambda$-modules $L$ of finite length.

Restricting still further, one arrives at a very satisfactory class of rings. Recall that for $(R, \mathfrak{m})$ a local ring, a finitely generated $R$-module $M$ is maximal Cohen-Macaulay $(M C M)$ if $\operatorname{depth} M=\operatorname{dim} R$. Equivalently, there is a system of parameters $x_{1}, \ldots, x_{d}$, with $d=\operatorname{dim} R$, which is an $M$-regular sequence. In the special case where $R$ is Gorenstein, this condition is equivalent to $\operatorname{Ext}_{R}^{i}(M, R)=0$ for all $i>0$.

Definition F.2. Let $(R, \mathfrak{m})$ be a local ring and $\Lambda$ a module-finite $R$-algebra. Say that $\Lambda$ is an $R$-order if $\Lambda$ is maximal Cohen-Macaulay as an $R$-module.

The terminology is imperfect: there are several other definitions of the word "order" in the literature, going back decades. Here we follow [Aus78]. See $\S \mathbb{K}$ for a connection to the classical theory of hereditary and classical orders over Dedekind domains.

Localization is still problematic, even for orders. In order to get a workable theory, a condition stronger than finite global dimension is sometimes needed.

Definition F.3. Let $R$ be a commutative ring and let $\Lambda$ be a module-finite $R$-algebra. Say that $\Lambda$ is non-singular if gldim $\Lambda_{\mathfrak{p}}=\operatorname{dim} R_{\mathfrak{p}}$ for every prime ideal $\mathfrak{p}$ of $R$.

Non-singular orders have a very satisfactory homological theory, especially over Gorenstein local rings. A non-singular order over a local ring satisfies a version of the AuslanderBuchsbaum Theorem [IR08, Proposition 2.3]: If $\Lambda$ is an $R$-order with gldim $\Lambda=d<\infty$, then for any $\Lambda$-module $M$ the equality $\operatorname{pd}_{\Lambda} M+\operatorname{depth} M=d$ holds. Furthermore, the following characterization of non-singularity holds for orders [IW10, Proposition 2.13].

Proposition F.4. Let $R$ be a CM ring with a canonical module $\omega$, and let $\Lambda$ be an $R$-order. Then the following are equivalent.

(i) $\Lambda$ is non-singular.

(ii) gldim $\Lambda_{\mathfrak{m}}=\operatorname{dim} R_{\mathfrak{m}}$ for all maximal ideals $\mathfrak{m}$ of $R$.

(iii) The finitely generated $\Lambda$-modules which are MCM as $R$-modules are precisely the finitely generated projective $\Lambda$-modules.

(iv) $\operatorname{Hom}_{R}(\Lambda, \omega)$ is a projective $\Lambda$-module and gldim $\Lambda<\infty$.

The definitions above represent an attempt to force classical algebraic geometry, or equivalently commutative algebra, to work over a class of non-commutative rings. Here is a different approach, more consonant with the idea of "categorical geometry." Rather than focusing attention on the rings, concentrate on an abelian or triangulated category $\mathrm{C}$, which 
we choose to think of as $\mathrm{Q} \operatorname{ch} X$ or $\mathrm{D}^{b}(\mathrm{Q} \operatorname{ch} X)$ for some space $X$ about which we say nothing further. In this approach, the space $X$ is nothing but a notational placeholder, and the geometric object is the category $C$.

This idea has had particular success in taking Serre's Theorem B.2 as a template and writing $D^{b}(Q \operatorname{ch} X)$ for a quotient category of the form tails $\Lambda=\operatorname{grmod} \Lambda /$ tors $\Lambda$. One thus obtains what is called non-commutative projective geometry. To describe these successes, let us make the following definition, based on the work of Geigle-Lenzing [GL87], Verëvkin [Ver92b, Ver92a], Artin-Zhang [AZ94] and Van den Bergh [VdB01].

Definition F.5. A quasi-scheme (over a field $k)$ is a pair $X=\left(X-\bmod , \mathscr{O}_{X}\right)$ where $X$-mod is a ( $k$-linear) abelian category and $\mathscr{O}_{X} \in X$-mod is an object. Two quasi-schemes $X$ and $Y$ are isomorphic (over $k$ ) if there exists a ( $k$-linear) equivalence $\mathrm{F}: X$-mod $\longrightarrow Y$-mod such that $\mathrm{F}\left(\mathscr{O}_{X}\right) \cong \mathscr{O}_{Y}$.

The obvious first example is that a (usual, commutative) scheme $X$ is a quasi-scheme ( $\operatorname{coh} X, \mathscr{O}_{X}$ ). For any ring $\Lambda$, commutative or not, one can define the affine quasi-scheme associated to $\Lambda$ to be $\operatorname{Spec} \Lambda:=(\Lambda$-mod, $\Lambda)$. One checks that if $R$ is commutative and $X=\operatorname{Spec} R$ is the usual prime spectrum, then the global section functor $\Gamma(X,-): \operatorname{coh} X \longrightarrow R$-mod induces an isomorphism of quasi-schemes $\left(\operatorname{coh} X, \mathscr{O}_{X}\right) \longrightarrow(R$-mod, $R)$.

The basic example of a quasi-scheme in non-commutative projective geometry is the following, which mimics the definition of tails from $\S \mathrm{B}$ precisely. Let $\Lambda$ be a noetherian graded algebra over a field $k$. For simplicity, assume that $A_{0}=k$. Let $\operatorname{GrMod} \Lambda$ and $\operatorname{grmod} \Lambda$ be the categories of graded $\Lambda$-modules, resp. finitely generated graded $\Lambda$-modules. Let Tors $\Lambda$, resp. tors $\Lambda$, be the subcategory of graded modules annihilated by $\Lambda_{\geqslant n}$ for $n \gg 0$. Then define the quotient categories

$$
\text { Tails } \Lambda=\operatorname{GrMod} \Lambda / \operatorname{Tors} \Lambda \quad \text { and } \quad \text { tails } \Lambda=\operatorname{grmod} \Lambda / \operatorname{tors} \Lambda \text {, }
$$

and set

$$
\operatorname{Proj} \Lambda=(\text { Tails } \Lambda, \mathscr{O}) \quad \text { and } \quad \operatorname{proj} \Lambda=(\operatorname{tails} \Lambda, \mathscr{O})
$$

where $\mathscr{O}$ is the image of $\Lambda$ in tails $\Lambda$. Call $\operatorname{Proj} \Lambda$ and $\operatorname{proj} \Lambda$ the (noetherian) projective quasischeme determined by $\Lambda$. The dimension of the projective quasi-scheme is GKdim $\Lambda-1$, where GKdim $\Lambda$ is the Gelfand-Kirillov dimension; this means $\operatorname{dim} \operatorname{proj} \Lambda$ is the polynomial rate of growth of $\left\{\operatorname{dim}_{k} \Lambda_{n}\right\}_{n \geqslant 0}$.

One can define sheaf cohomology $H^{j}$ (Tails $\left.\Lambda,-\right)$ in Tails $\Lambda$ to directly generalize the commutative definition. In particular the global sections functor is $\Gamma(-)=\operatorname{Hom}_{\text {Tails } \Lambda}(\mathscr{O},-)$. For $M$ in Tails $\Lambda$, then, one would like versions of two basic results in algebraic geometry: Serrefiniteness $\left(H^{j}\right.$ (Tails $\left.\Lambda, M\right)=0$ for $\left.j \gg 0\right)$ and Serre-vanishing $\left(H^{j}\right.$ (Tails $\left.\Lambda, M(i)\right)=0$ for all $j \geqslant 1$ and $i \gg 0$ ). These results turn out only to be true under a technical condition called $\chi$ (see [AZ94]), which is automatic in the commutative case. There is also an analogue of Serre's Theorem B.2 due to Artin and Van den Bergh [AVdB90], which gives the same sort of purely algebraic description of $\mathrm{Q} \operatorname{ch} X$ as Tails $\Lambda$, where $\Lambda$ is defined to be a twisted homogeneous coordinate ring. For details, see [SVdB01].

The classification of projective quasi-schemes of small dimension, i.e. categories of the form tails $\Lambda$ where $\Lambda$ is a graded algebra with small rate of growth, is an ongoing program. The case of non-commutative curves (where $\operatorname{dim}_{k} A_{n}$ grows linearly) was completed by Artin and Stafford [AS95]. There is a conjectural classification of non-commutative surfaces due to Artin, but it is still open. The important special case of non-commutative projective planes, 
that is, tails $\Lambda$ where $\Lambda$ is a so-called Artin-Schelter regular algebra of Gelfand-Kirillov dimension 3 with Hilbert series $(1-t)^{-3}$, has been completely understood [AS87, ATVdB90, ATVdB91, VdB87, BP93].

\section{G. Resolutions of Singularities}

So far I have considered only "absolute" situations, that is, constructions applied to individual rings or categories in isolation. In the sections to come, I will want to understand certain relative situations, particularly analogues of resolutions of singularities. In this $\S$ I collect a few definitions and facts about resolutions of singularities, for easy reference later. Begin with the definition.

Definition G.1. Let $X$ be an algebraic variety over a field $k$. A resolution of singularities of $X$ is a proper, birational morphism $\pi: \widetilde{X} \longrightarrow X$ with $\widetilde{X}$ a non-singular algebraic variety.

Resolutions of singularities are also sometimes called "smooth models," indicating that the non-singular variety $\widetilde{X}$ is not too different from $X$ : the map is an isomorphism on a dense open set and is proper, hence surjective. For curves, construction of resolution of singularities is easy, as every irreducible curve is birational to a unique smooth projective curve, namely the normalization (see \$L). For surfaces, resolutions of singularities still exist in any characteristic, but now an irreducible surface is birational to infinitely many smooth surfaces. This observation is the beginning of the minimal model program, cf. $\S \mathrm{H}$.

Of course existence of resolutions of singularities in any dimension is a theorem due to Hironaka for $k$ an algebraically closed field of characteristic zero; in this case the morphism $\pi: \widetilde{X} \longrightarrow X$ can be taken to be an isomorphism over the smooth locus of $X$, and even to be obtained as a sequence of blowups of non-singular subvarieties of the singular locus followed by normalizations. We will not need this.

As an aside, I mention here that a proper map between affine schemes is necessarily finite [Har77, Ex. II.4.6]. It follows immediately that a resolution of singularities of a singular normal affine scheme is never an affine scheme.

Our other definitions require the canonical sheaf of a singular variety. The canonical sheaf $\omega_{Y}$ of a smooth variety $Y$ has already appeared, as the sheaf of top differential forms $\wedge^{\operatorname{dim} Y} \Omega_{Y}$ (see the discussion before Theorem D.1). If $Y$ is merely normal, then define $\omega_{Y}$ to be $j_{*} \omega_{Y_{\text {reg }}}$, where $j$ is the open immersion $Y_{\text {reg }} \hookrightarrow Y$ of the smooth locus. When $Y$ is CohenMacaulay, $\omega_{Y}$ is also a dualizing sheaf [Har77, III.7]; in other words, if the local rings of $Y$ are CM, then the stalks of $\omega_{Y}$ are canonical modules in the sense of [HK71]. Similarly, $\omega_{Y}$ is an invertible sheaf (line bundle) if and only if $Y$ is Gorenstein. The Weil divisor $K_{Y}$ such that $\omega_{Y}=\mathscr{O}_{Y}\left(K_{Y}\right)$ is called the canonical divisor.

The behavior of the canonical sheaf/divisor under certain morphisms is of central interest. For example, the Grauert-Riemenschneider Vanishing theorem describes the higher direct images of $\omega$.

Theorem G.2 (GR Vanishing [GR70]). Let $\pi: \widetilde{X} \longrightarrow X$ be a resolution of singularities of a variety $X$ over $\mathbb{C}$. Then $\mathbf{R}^{i} \pi_{*} \omega_{\tilde{X}}=0$ for all $i>0$.

Now I come to a pair of words which will be central for the rest of the article.

Definition G.3. Let $\pi: \widetilde{X} \longrightarrow X$ be a resolution of singularities of a normal variety $X$. 
(i) Say that $\pi$ is a rational resolution if $\mathbf{R}^{i} \pi_{*} \mathscr{O}_{\widetilde{X}}=0$ for $i>0$. Equivalently, since $X$ is normal, $\mathbf{R} \pi_{*} \mathscr{O}_{\widetilde{X}}=\mathscr{O}_{X}$. In this case $X$ is said to have rational singularities.

(ii) Say that $\pi$ is a crepant $^{1}$ resolution if $\pi^{*} \omega_{X}=\omega_{\widetilde{X}}$.

Crepancy is a condition relating the two ways of getting a sheaf on $\widetilde{X}$ from one on $X$, namely via Hom and via $\otimes$. To get an idea what this condition is, consider a homomorphism of CM local rings $R \longrightarrow S$ such that $S$ is a finitely generated $R$-module. Let $\omega_{R}$ be a canonical module for $R$. Then one knows that the "co-induced" module $\operatorname{Ext}_{R}^{t}\left(S, \omega_{R}\right)$, where $t=\operatorname{dim} R-$ $\operatorname{dim} S$, is a canonical module for $S$ [BH93, 3.3.7]. The "induced" module $S \otimes_{R} \omega_{R}$ is not necessarily a canonical module. Back in the geometric world, $\pi^{*} \omega_{\text {Spec } R}$ corresponds to $S \otimes_{R}$ $\omega_{R}$, so the assumption that this is equal to $\omega_{S}$ is locally a condition of the form $\operatorname{Ext}_{R}^{t}\left(S, \omega_{R}\right) \cong$ $S \otimes_{R} \omega_{R}$. When $X$ is Gorenstein, i.e. $\omega_{X} \cong \mathscr{O}_{X}$, a crepant resolution $\widetilde{X}$ is also Gorenstein.

One of the main motivations for considering crepant resolutions of singularities comes from the study of Calabi-Yau varieties, which in particular have trivial canonical sheaves. In this case, if one wants a resolution $\pi: \widetilde{X} \longrightarrow X$ in which $\widetilde{X}$ is also Calabi-Yau, then $\pi$ needs to be crepant.

A small resolution, that is, one for which the exceptional locus has codimension at least two, is automatically crepant. This is a very useful sufficient condition.

The next proposition follows from GR vanishing [KKMSD73, p. 50].

Proposition G.4. Let $X$ be a complex algebraic variety and let $\pi: \widetilde{X} \longrightarrow X$ be a resolution of singularities.

(i) $X$ has rational singularities if and only if $X$ is $C M$ and $\pi_{*} \omega_{\tilde{X}}=\omega_{X}$.

(ii) If $X$ is Gorenstein and has a crepant resolution of singularities, then $X$ has rational singularities.

Not every rational singularity has a crepant resolution. Here are two examples.

Example G.5. Let $R$ be the diagonal hypersurface ring $\mathbb{C}[x, y, z, t] /\left(x^{3}+y^{3}+z^{3}+t^{2}\right)$. Then $R$ is quasi-homogeneous with the variables given weights $2,2,2$, and 3 . The $a$-invariant of $R$ is thus $6-(2+2+2+3)=-3<0$, and $R$ has rational singularities by Fedder's criterion [Hun96. Example 3.9]. However, Lin [Lin02] shows that a diagonal hypersurface defined by $x_{0}^{r}+x_{1}^{d}+$ $\cdots+x_{d}^{d}$ has a crepant resolution of singularities if and only if $r$ is congruent to 0 or $1 \bmod d$.

Example G.6. Quotient singularities $X=Y / G$, where $Y$ is smooth and $G$ is a finite group of automorphisms, have rational singularities [Vie77]. Consider quotient singularities $\mathbb{C}^{n} / G$, where $G \subset \operatorname{SL}(n, \mathbb{C})$ is finite. These are by [Wat74] the Gorenstein quotient singularities.

If $n=2$, the results are the rational double points, also known as Kleinian singularities or Du Val singularities, which are the quotient singularities $X=\mathbb{C}^{2} / G=\operatorname{Spec}\left(\mathbb{C}[u, v]^{G}\right)$, where $G \subset \mathrm{SL}(2, \mathbb{C})$ is a finite subgroup. These are also described as $\mathrm{ADE}$ hypersurface rings

\footnotetext{
${ }^{1}$ Obligatory comment on the terminology: the word "crepant" is due to Miles Reid. He describes it [Rei00, p. 330] as a pun meaning "non-discrepant", in that the discrepancy divisor $K_{\tilde{X}}-\pi^{*} K_{X}$ vanishes.
} 
$\mathbb{C}[x, y, z] /(f(x, y, z))$ with explicit equations as follows.

$$
\begin{array}{ll}
\left(A_{n}\right): & x^{2}+y^{n+1}+z^{2}, \quad n \geqslant 1 \\
\left(D_{n}\right): & x^{2} y+y^{n-1}+z^{2}, \quad n \geqslant 4 \\
\left(E_{6}\right): & x^{3}+y^{4}+z^{2} \\
\left(E_{7}\right): & x^{3}+x y^{3}+z^{2} \\
\left(E_{8}\right): & x^{3}+y^{5}+z^{2}
\end{array}
$$

For these singularities, a crepant resolution always exists and is unique. In fact, a normal affine surface singularity $R$ over $\mathbb{C}$ admits a crepant resolution if and only if every local ring of $R$ is (at worst) a rational double point. I will return to the rational double points in $\$ J$ below.

If $n=3, \mathbb{C}^{3} / G$ always has a crepant resolution as well, though they are no longer unique, thanks to the existence of flops (see the next $\S$ ). There is a classification of the finite subgroups of $\mathrm{SL}(3, \mathbb{C})$ up to conjugacy, and existence of crepant resolutions was verified on a case-by-case basis by Markushevich [Mar97], Roan [Roa94, Roa96], Ito [Ito95b, Ito95a], and Ito-Reid [IR96]. See Theorem J.8 below for a unified statement.

For $n \geqslant 4$, quotient singularities need not have crepant resolutions of singularities. For example, the quotient of $\mathbb{C}^{4}$ by the involution $(x, y, z, w) \mapsto(-x,-y,-z,-w)$ admits no crepant resolution [Rei02, Example 5.4].

\section{H. THE MiNimAl MODEL PROGRAM}

A key motivation for categorical desingularizations in general, and non-commutative crepant resolutions in particular, is the minimal model program of Mori and Reid. This is an attempt to find a unique "best" representative for the birational equivalence class of any algebraic variety. For curves, this is obvious, since there is in each equivalence class a unique smooth projective representative.

It is also the case that every surface is birationally equivalent to a smooth projective surface, but now matters are complicated by the fact that the blowup of a smooth surface at a point is again smooth. However, every birational morphism of surfaces factors as a sequence of blowups, so must have a (-1)-curve, that is, a rational curve $C \cong \mathbb{P}^{1}$ with selfintersection -1 , lying over a smooth point. One can compute that if $C$ is a (-1)-curve on a surface $X$, then $K_{X} \cdot C=-1$, where $K_{X}$ is the canonical divisor.

By Castelnuovo's criterion, a (-1)-curve can always be blown down, essentially undoing the blowup. The algorithm for obtaining a minimal model is thus to contract all the $(-1)$ curves, and one obtains the classification of minimal models for surfaces [Har77, V.5]: the result of the algorithm is a smooth projective surface $S$ which is either $\mathbb{P}^{2}$, a ruled surface over a curve (the "Fano" case), or such that $K_{S} \cdot C \geqslant 0$ for every curve $C$ in $S$. In this last case say that $K_{S}$ is nef.

The minimal model program is a framework for extending this simple-minded algorithm to one that will work for threefolds and higher-dimensional varieties. The theory turns out to be much richer, in part because it turns out that one must allow minimal models to be a little bit singular. Here "a little bit" means in codimension $\geqslant 2$. Precisely, a projective variety $X$ is a minimal model if every birational map $Y \rightarrow X$ is either a contraction of a divisor to a set of codimension at least two, or is an isomorphism outside sets of codimension 
at least two [Kol07]. There are compelling reasons to allow singular minimal models; for example, there exists a three-dimensional smooth variety which is not birational to any smooth variety with nef canonical divisor [MOb]. Mori and Reid realized that this meant minimal models need not be smooth; they can be taken to be terminal instead.

I won't worry about the technical definitions of terminal and canonical singularities here, but only illustrate with a class of examples. A diagonal hypersurface singularity defined by $x_{0}^{a_{0}}+x_{1}^{a_{1}}+\cdots+x_{d}^{a_{d}}$ is

(i) canonical if and only if $a_{1}+\cdots+a_{d}>1$, and

(ii) terminal if and only if $a_{1}+\cdots+a_{d}>1+\frac{1}{\operatorname{lcm}\left(a_{i}\right)}$.

For Gorenstein singularities, canonical singularities are the same as rational singularities, so Proposition G.4(ii) says that the existence of a crepant resolution implies canonical singularities.

In this language, a projective variety $X$ is a minimal model if and only if it is $\mathbb{Q}$-factorial (i.e. the divisor class group of every local ring is torsion), has nef canonical divisor, and has terminal singularities.

In dimension two, minimal models are unique up to isomorphism by definition. Terminal surface singularities are smooth, and the canonical surface singularities are the rational double points of Example G.6 [Kol89b, (2.6.2)].

In dimension three, terminal singularities are well-understood, cf. [Rei83] or [Kol89b, 2.7]. The Gorenstein ones are precisely the isolated compound $\mathrm{Du}$ Val (cDV) singularities. (Recall that a cDV singularity is a hypersurface defined by $f(x, y, z)+\operatorname{tg}(x, y, z, t)$, where $f$ is a simple singularity as in (G.1) and $g$ is arbitrary.) However, minimal models of threefolds are no longer unique [Cor04]. Here is the simplest example.

Example H.1 (The "classic flop"). Let $X$ be the three-dimensional $\left(A_{1}\right)$ singularity over $\mathbb{C}$, so $X=\operatorname{Spec} \mathbb{C}[u, v, x, y] /(u v-x y)$. Consider the blowup $f: Y \longrightarrow X$ of the plane $u=x=0$. It's easy to check that $Y$ is smooth, and that $f: Y \longrightarrow X$ is a birational map which contracts a line $L \cong \mathbb{P}^{1}$ to the origin. Thus $f$ is a small resolution, whence crepant. Furthermore $Y$ is a minimal model.

One could also have considered the plane $u=y=0$ and its blowup $f^{\prime}: Y^{\prime} \longrightarrow X$. Symmetrically, $Y^{\prime}$ is smooth, $f^{\prime}$ contracts a line $L^{\prime} \cong \mathbb{P}^{1}$ and is crepant, and $Y^{\prime}$ is a minimal model.

The resolutions $Y$ and $Y^{\prime}$ are almost indistinguishable, but they are not isomorphic over $X$. One can check that the birational transforms of the plane $u=x=0$ to $Y$ and $Y^{\prime}$ have intersection number +1 with $L$ and -1 with $L^{\prime}$.

On the other hand, the induced birational map $\varphi: Y \rightarrow Y^{\prime}$ is an isomorphism once one removes $L$ from $Y$ and $L^{\prime}$ from $Y^{\prime \prime}$. This $\varphi$ is called a (or "the classic") flop. It is also sometimes called the "Atiyah flop" after [Ati58], though Reid traces it back through work of Zariski in the 1930s, and assigns it a birthdate of around 1870 . 
Let $Z$ be the blowup of the origin of $X$. Then $Z$ is in fact the closed graph of $\varphi$ and there is a diagram

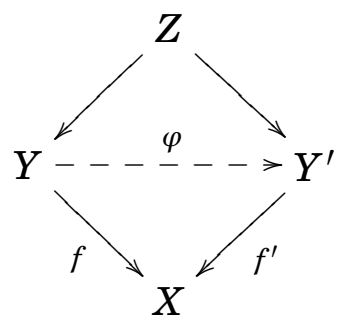

The exceptional surface of $Z \longrightarrow X$ is the quadric $Q=\mathbb{P}^{1} \times \mathbb{P}^{1}$, which is cut out by two families of lines. The lines $L$ and $L^{\prime}$ are the contractions of $Q$ along these two rulings, and conversely $Q$ is the blowup of $L \subset Y$, resp. $L^{\prime} \subset Y^{\prime}$.

The next definition is a special case of the usual definition of a flop [KM98, 6.10] (in general, one need not assume $Y$ and $Y^{\prime}$ are smooth, nor that $X$ is Gorenstein).

Definition H.2. Let $Y$ and $Y^{\prime}$ be smooth projective varieties. A birational map $\varphi: Y \rightarrow Y^{\prime}$ is a flop if there is a diagram

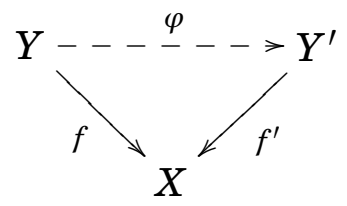

where $X$ is a normal projective Gorenstein variety, $f$ and $f^{\prime}$ are small resolutions of singularities, and there is a divisor $D$ on $Y$ such that, if $D^{\prime}$ is the strict transform of $D$ on $Y^{\prime}$, then $-D^{\prime}$ is ample.

Say $\varphi: Y \rightarrow Y^{\prime}$ is a generalized flop if for some (equivalently, for every) diagram

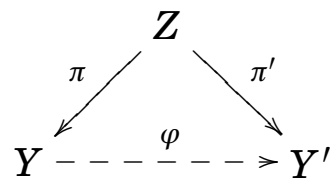

with $Z$ smooth, there is an equality $\pi^{*} K_{Y}=\pi^{\prime *} K_{Y^{\prime}}$.

It is known that the existence of a crepant resolution forces canonical singularities, so that in particular if $X$ participates in a flop as above, it has canonical singularities. On the other hand, if $X$ is $\mathbb{Q}$-factorial and has terminal singularities, then it can have no crepant resolution of singularities [Kol89a, Corollary 4.11] (this is one explanation of the name "terminal").

Bondal and Orlov [BO02] observed that one ingredient of the minimal model program, namely the blowup $\widetilde{X}$ of a smooth variety $X$ at a smooth center, induces a fully faithful functor on derived categories $\mathrm{D}^{b}(\operatorname{coh} X) \longrightarrow \mathrm{D}^{b}(\operatorname{coh} \widetilde{X})$. They propose that each of the operations of the program should induce such fully faithful embeddings. In particular, they make the following conjecture.

Conjecture H.3 (Bondal-Orlov). For any generalized flop $\varphi: Y \rightarrow \rightarrow Y^{\prime}$ between smooth varieties, there is an equivalence of triangulated categories $\mathrm{F}: \mathrm{D}^{b}\left(\operatorname{coh} Y^{\prime}\right) \longrightarrow \mathrm{D}^{b}(\operatorname{coh} Y)$. 
Notice that even though there always exists the natural Fourier-Mukai type functor $\mathbf{R} \pi_{*} \mathbf{L} \pi^{\prime *}(-): \mathrm{D}^{b}\left(\operatorname{coh} Y^{\prime}\right) \longrightarrow \mathrm{D}^{b}(\operatorname{coh} Y)$, this is known not to be fully faithful in general, so some new idea is needed.

Bondal and Orlov proved Conjecture $\mathrm{H.3}$ in some special cases in dimension three, and Bridgeland [Bri02] gave a complete proof for threefolds. Here is Bridgeland's result.

Theorem H.4 (Bridgeland). Let $X$ be a projective complex threefold with terminal singularities. Let $f: Y \longrightarrow X$ and $f^{\prime}: Y^{\prime} \longrightarrow X$ be crepant resolutions of $X$. Then $\mathrm{D}^{b}(\operatorname{coh} Y) \simeq$ $\mathrm{D}^{b}\left(\operatorname{coh} Y^{\prime}\right)$.

The equivalence in this theorem is a Fourier-Mukai type functor of the form $\mathbf{R} f_{*}(\mathscr{P} \otimes$ $\left.f^{\prime *}(-)\right)$, where $\mathscr{P}$ is a well-chosen object of $D^{b}\left(\operatorname{coh}\left(Y \times_{X} Y^{\prime}\right)\right)$. In fact the construction of $\mathscr{P}$ is very difficult and is the heart of the proof.

\section{CAtegorical Desingularizations}

Now let us combine the philosophical ramblings of $\S \mathrm{F}$ with the concrete problems of $\S \S \mathrm{G}$ and $\mathrm{H}$. Treating commutative and non-commutative varieties-in the form of their derived categories-on equal footing, one can entertain the notion of a resolution of a commutative algebraic variety by a non-commutative one. Bondal and Orlov [BO02] seem to have been the first to articulate such a possibility in pure mathematics. Other authors have considered modified or specialized versions, e.g. [Bez06, Kuz08, Lun10].

To begin, let us consider resolutions of singularities from a categorical point of view. Let $X$ be a normal algebraic variety, and let $\pi: \widetilde{X} \longrightarrow X$ be a resolution of singularities. There are two natural functors between derived categories, namely the derived pushforward $\mathbf{R} \pi_{*}: \mathrm{D}^{b}(\operatorname{coh} \widetilde{X}) \longrightarrow \mathrm{D}^{b}(\operatorname{coh} X)$ and the derived pullback $\mathbf{L} \pi^{*}: \mathrm{D}(\operatorname{coh} X) \longrightarrow \mathrm{D}(\operatorname{coh} \widetilde{X})$. The derived pullback may not take bounded complexes to right-bounded ones, so does not generally give a functor on $\mathrm{D}^{b}$. One could restrict $\mathbf{L} \pi^{*}$ to the perfect complexes over $X$ and write instead $\mathbf{L} \pi^{*}: \mathrm{D}^{\text {perf }}(\operatorname{coh} X) \longrightarrow \mathrm{D}^{\text {perf }}(\operatorname{coh} \widetilde{X})=\mathrm{D}^{b}(\operatorname{coh} X)$.

The pullback and pushforward form an adjoint pair. If $X$ is assumed to have rational singularities, much more can be said. For an object $\mathscr{E}$ in $\mathrm{D}^{b}(\operatorname{coh} \widetilde{X})$ and a perfect complex $\mathscr{P}$ over $X$, the derived projection formula gives

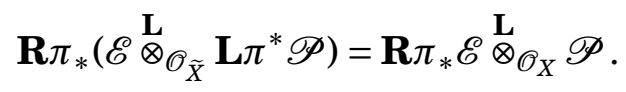

In particular, setting $\mathscr{E}=\mathscr{O}_{\widetilde{X}}$ and taking into account $\mathbf{R} \pi_{*} \mathscr{O}_{\widetilde{X}}=\mathscr{O}_{X}$, this yields

$$
\mathbf{R} \pi_{*} \mathbf{L} \pi^{*} \mathscr{P}=\mathscr{P}
$$

for every perfect complex $\mathscr{P}$ in $\mathrm{D}^{b}(\operatorname{coh} X)$. Otherwise said, $\mathbf{R} \pi_{*}: \mathrm{D}^{b}(\operatorname{coh} \widetilde{X}) \longrightarrow \mathrm{D}^{b}(\operatorname{coh} X)$ identifies the target with the quotient of the source by the kernel of $\mathbf{R} \pi_{*}$. Bondal and Orlov propose to take this as a template:

Definition I.1 (Bondal-Orlov). A categorical desingularization of a triangulated category $D$ is an abelian category $C$ of finite homological dimension and a triangulated subcategory $\mathrm{K}$ of $\mathrm{D}^{b}(\mathrm{C})$, closed under direct summands, such that $\mathrm{D}^{b}(\mathrm{C}) / \mathrm{K} \simeq \mathrm{D}$.

One problem with this definition is the assumption that $C$ have finite homological dimension. As observed in $\$ \mathrm{~F}$, this is a very weak condition when $C$ is the category of modules over a non-commutative ring. There are a number of proposals for a better-that is, more 
restrictive-notion of smoothness for a (triangulated) category, but as far as I can tell, no consensus on a best candidate [KS09, TV08, Kuz08, Lun10].

As an aside, I note here that the condition for $\pi: \widetilde{X} \longrightarrow X$ to be crepant can be translated into categorical language as "the right adjoint functor $\pi^{!}$, which is locally represented by $\operatorname{Hom}_{\mathscr{O}_{X}}\left(\mathscr{O}_{\widetilde{X}},-\right)$, is isomorphic to $\pi^{*}$." We won't need this.

Let us reconsider Example $\mathrm{H} .1$ from the point of view of categorical geometry. This can be thought of as a warmup for $\$ \mathrm{Q}$.

Example I.2. Set $R=\mathbb{C}[u, v, x, y] /(u v-x y)$, so that $X=\operatorname{Spec} R$ is the three-dimensional ordinary double point as in Example H.1. Let $I=(u, x)$ and $I^{\prime}=(u, y)$. Then in fact $I^{\prime}=I^{-1}=$ $I^{*}=\operatorname{Hom}_{R}(I, R)$ is the dual of $I$. Notice too that $\operatorname{End}_{R}(I)=R$, either by direct computation or by Theorem L.1 below.

Let $f: Y \longrightarrow X$ and $f^{\prime}: Y^{\prime} \longrightarrow X$ be the blowups of $I$ and $I^{\prime}$ as before. On $Y$, consider the locally free sheaf $\mathscr{E}=\mathscr{O}_{Y} \oplus \mathscr{O}_{Y}(1)$, which is the pullback of $\mathscr{O}_{X} \oplus \mathscr{I}$, where $\mathscr{I}$ is the ideal sheaf of $I$. Straightforward calculations (or see $\$ Q$ ) show that $\mathscr{E}$ is a tilting bundle on $Y$ (Definition $(\overline{\mathrm{D} .2})$, and hence $\mathbf{R H o m}_{\mathscr{O}_{Y}}(\mathscr{E},-): \mathrm{D}^{b}(\operatorname{coh} Y) \longrightarrow \mathrm{D}^{b}(\Lambda$-mod) is an equivalence, where $\Lambda=\operatorname{End}_{\mathscr{O}_{Y}}(\mathscr{E})$. Furthermore, we have

$$
\Lambda \cong f_{*} \mathscr{E} n d_{\mathscr{O}_{Y}}(\mathscr{E})=\operatorname{End}_{R}(R \oplus I),
$$

which can also be written as a block-matrix ring

$$
\Lambda=\left(\begin{array}{cc}
R & I \\
I^{-1} & \operatorname{End}_{R}(I)=R
\end{array}\right) .
$$

The induced functor $\mathrm{D}^{b}(\Lambda-\bmod ) \longrightarrow \mathrm{D}^{b}(\operatorname{coh} X)$ is then obviously a categorical desingularization.

Repeating the construction above with $\mathscr{E}^{\prime}=\mathscr{O}_{Y^{\prime}} \oplus \mathscr{O}_{Y^{\prime}}(1)$ on $Y^{\prime}$, one obtains $\Lambda^{\prime}=\operatorname{End}{ }_{R}(R \oplus$ $\left.I^{\prime}\right)$. But since $I^{\prime}=I^{-1}, \Lambda^{\prime}$ is isomorphic to $\Lambda$. This implies equivalences

$$
\mathrm{D}^{b}(\operatorname{coh} Y) \simeq \mathrm{D}^{b}(\Lambda-\bmod ) \simeq \mathrm{D}^{b}\left(\operatorname{coh} Y^{\prime}\right) .
$$

Inspired by the example above and others from the minimal model program, Bondal and Orlov expect that for a singular variety $X$, the category $\mathrm{D}^{b}(\operatorname{coh} X)$ should have a minimal categorical desingularization, i.e. one embedding in any other. Such a category would be unique up to derived equivalence. They propose in particular the following conjecture.

Conjecture I.3 (Bondal-Orlov [BOB2] $)$. Let X be a complex algebraic variety with canonical singularities and let $f: Y \longrightarrow X$ be a finite morphism with $Y$ smooth. Then $\mathscr{A}=$ $\mathscr{E} n d_{\mathscr{O}_{X}}\left(f_{*} \mathscr{O}_{Y}\right)$ gives a minimal categorical desingularization, in the sense that $\mathscr{A}$-mod has finite global dimension and if $\widetilde{X} \longrightarrow X$ is any other resolution of singularities of $X$, then there exists a fully faithful embedding $\mathrm{D}^{b}(\mathscr{A}$-mod $) \longrightarrow \mathrm{D}^{b}(\operatorname{coh} \widetilde{X})$. Moreover, if $\widetilde{X} \longrightarrow X$ is crepant, then the embedding is an equivalence.

In the next $\S$ I will consider another family of examples providing strong evidence for this conjecture.

\section{J. Example: the MCKay CORRESPONDENCE}

In this $\S$ I sketch a main motivating example, already foreshadowed in Example G.6. The finite subgroups of $\mathrm{SL}(2, \mathbb{C})$ were carefully studied by Klein in the 1880 s, and the resolutions 
of the corresponding singularities $\mathbb{C}^{2} / G=\operatorname{Spec} \mathbb{C}[u, v]^{G}$ were understood by Du Val in the 1930s. The structure of the resolution faithfully reflects the representation theory of the group $G$, as observed by McKay [McK80], and the correspondence naturally extends to the reflexive modules over the (completed) coordinate ring $\mathbb{C} \llbracket u, v \rrbracket^{G}$. Even more, there is a natural resolution of singularities of the quotient singularity, built from the group $G$, which is derived equivalent to a certain non-commutative ring built from these reflexive modules. Thus the group $G$ already knows the geometry of $\mathbb{C}^{2} / G$ and its resolution of singularities.

This $\S$ is about this circle of ideas, which together go by the name "McKay correspondence." I consider first, more generally, finite subgroups $G \subset \operatorname{GL}(n, k)$ with $n \geqslant 2$ and $k$ a field of characteristic relatively prime to $|G|$. Then I specialize to $n=2$ and subgroups of SL, where the strongest results hold. See [LW] or [Yos90] for proofs.

Let $S=k \llbracket x_{1}, \ldots, x_{n} \rrbracket$ be a power series ring over an algebraically closed field $k$ with $n \geqslant 2$. Let $G \subset \operatorname{GL}(n, k)$ be a finite subgroup with order invertible in $k$. Make $G$ act on $S$ by linear changes of variables, and set $R=S^{G}$, the ring of invariants. The ring $R$ is noetherian, local, and complete, of dimension $n$. It is even CM by the Hochster-Eagon theorem [HE71]. Furthermore, $S$ is a module-finite $R$-algebra, and is a maximal Cohen-Macaulay $R$-module.

The central character in the story is the skew, or twisted, group algebra $S \# G$. As an $S$ module, $S \# G$ is free on the elements of $G$, and the product of two elements $s \cdot \sigma$ and $t \cdot \tau$, with $s, t \in S$ and $\sigma, \tau \in G$, is defined by $(s \cdot \sigma)(t \cdot \tau)=s \sigma(t) \cdot \sigma \tau$. Thus moving $\sigma$ past $t$ "twists" the ring element.

Left modules over $S \# G$ are precisely $S$-modules with a compatible action of $G$, and one computes $\operatorname{Hom}_{S \# G}(M, N)=\operatorname{Hom}_{S}(M, N)^{G}$ for $S \# G$-modules $M$ and $N$. Since the order of $G$ is invertible, taking invariants is an exact functor, whence $\operatorname{Ext}_{S \# G}^{i}(M, N)=\operatorname{Ext}_{S}^{i}(M, N)^{G}$ for all $i>0$ as well. It follows that an $S \# G$-module $P$ is projective if and only if it is free over $S$. This, together with a moment's contemplation of the ( $G$-equivariant) Koszul complex over $S$ on $x_{1}, \ldots, x_{n}$, gives the following observation.

Proposition J.1. The twisted group ring $S \# G$, where $S=k \llbracket x_{1}, \ldots, x_{n} \rrbracket$ and $G$ is a finite group of linear automorphisms of $S$ with order invertible in $k$, has finite global dimension equal to $n$.

The "skew" multiplication rule in $S \# G$ is cooked up precisely so that the homomorphism $\gamma: S \# G \longrightarrow \operatorname{End}_{R}(S)$, defined by $\gamma(s \cdot \sigma)(t)=s \sigma(t)$, is a ring homomorphism extending the group homomorphism $G \longrightarrow \operatorname{End}_{R}(S)$ defining the action of $G$ on $S$. In general, $\gamma$ is neither injective nor surjective, but under an additional assumption on $G$, it is both. Recall that a pseudo-reflection is an element $\sigma \in \mathrm{GL}(n, k)$ of finite order which fixes a hyperplane.

Theorem J.2 (Auslander [Aus62, Aus86b]). Let $S=k \llbracket x_{1}, \ldots, x_{n} \rrbracket, n \geqslant 2$, let $G \subset \mathrm{GL}(n, k)$ be a finite group acting on $S$, and assume $|G|$ is invertible in $S$. Set $R=S^{G}$. If $G$ contains no nontrivial pseudo-reflections then the homomorphism $\gamma: S \# G \longrightarrow \operatorname{End}_{R}(S)$ is an isomorphism.

Consequently, in this case $\operatorname{End}_{R}(S)$ has finite global dimension and as an $R$-module is isomorphic to a direct sum of copies of $S$, so in particular is a MCM R-module.

The condition that $G$ contain no non-trivial pseudo-reflections is equivalent to the extension $R \hookrightarrow S$ being unramified in codimension one [Yos90, Lemma 10.7].

Let $\varrho: G \longrightarrow \mathrm{GL}(W)$ be a representation of $G$ on the finite-dimensional $k$-vector space $W$. Then $S \otimes_{k} W$, with the diagonal action of $G$, is a finitely generated $S \# G$-module. It is free over $S$, whence projective over $S \# G$. The submodule of fixed points, $M_{\varrho}=\left(S \otimes_{k} W\right)^{G}$, is 
naturally an $R$-module. If $\varrho$ is irreducible, then one can show that $M_{\varrho}$ is a direct summand of $S$ as an $R$-module. Conversely, given any $R$-direct summand of $S$, the corresponding idempotent in $\operatorname{End}_{R}(S)$ defines an $S \# G$-direct summand $P$ of $S \# G$, whence a representation $P /\left(x_{1}, \ldots, x_{n}\right) P$ of $G$.

Corollary J.3. These operations induce equivalences between the categories $\operatorname{add}_{R}(S)$ of $R$ direct summands of $S$, add $\operatorname{End}_{R}(S)$ of finitely generated projective $\operatorname{End}_{R}(S)$-modules, add $S \# G$ of finitely generated projective $S \# G$-modules, and $\operatorname{rep}_{k} G$ of finite-dimensional representations of $G$.

As a final ingredient, define a quiver from the data of the representation theory of $G$, or equivalently-given the correspondences above-of the $R$-module structure of $S$.

Definition J.4 (McKay [McK80]). The McKay quiver of $G \subset \mathrm{GL}(n, k)$ has vertices $\varrho_{0}, \ldots, \varrho_{d}$, a complete set of the non-isomorphic irreducible $k$-representations of $G$, with $\varrho_{0}$ the trivial irrep. Denote by $\omega$ the given $n$-dimensional representation of $G$ as a subgroup of $\operatorname{GL}(n, k)$. Then draw $m_{i j}$ arrows $\varrho_{i} \longrightarrow \varrho_{j}$ if the multiplicity of $\varrho_{i}$ in $\varrho \otimes_{k} \varrho_{j}$ is equal to $m_{i j}$.

Now let us specialize to the case $n=2$. Here the MCM $R$-modules are precisely the reflexive ones. This case is unique thanks to the following result, which fails badly for $n \geqslant 3$.

Lemma J.5 (Herzog [ [Her78]). Let $S=k \llbracket u, v \rrbracket$, let $G \subset \mathrm{GL}(2, k)$ be a finite group of order invertible in $k$, and let $R=S^{G}$. Then every finitely generated reflexive $R$-module is a direct summand of a direct sum of copies of $S$ as an $R$-module. In particular, the MCM $R$-modules coincide with $\operatorname{add}_{R}(S)$, and there are only finitely many indecomposable ones.

The one-one correspondences that hold for arbitrary $n$ can thus be augmented in dimension two, giving a correspondence between the irreducible representations of $G$ and the indecomposable MCM $R$-modules.

Specialize one last time, to assume now that $G \subset \mathrm{SL}(2, k)$. Note that then $G$ automatically contains no non-trivial pseudo-reflections. Furthermore, $R=S^{G}$ is Gorenstein by a result of Watanabe [Wat74]; in fact, it is classical [Kle93] that Spec $R$ embeds as a hypersurface in $k^{3}$, so $R \cong k \llbracket x, y, z \rrbracket / f(x, y, z)$ for some polynomial $f$. As long as $k$ has characteristic not equal to 2,3 , or 5 , the polynomials arising are precisely the ADE polynomials of (G.1) defining the rational double points.

The rational double points are distinguished among normal surface singularities by the fact that their local rings have unique crepant resolutions of singularities, which are the minimal resolutions of singularities. They are particularly easy to compute, being achieved by a sequence of blowups of points (no normalization required). The preimage of the singular point is a bunch of rational curves $E_{1}, \ldots, E_{n}$ on the resolution. These curves define the dual graph of the desingularization: it has for vertices the irreducible components $E_{1}, \ldots, E_{n}$, with an edge joining $E_{i}$ to $E_{j}$ if $E_{i} \cap E_{j} \neq 0$. This graph is related to the other data as follows.

Theorem J.6 (Classical McKay Correspondence). Let $k$ be an algebraically closed field of characteristic not 2,3 , or 5 , and let $G \subset \mathrm{SL}(2, k)$ be a finite subgroup of order invertible in $k$. Set $S=k \llbracket u, v \rrbracket$, with a natural linear action of $G$, set $R=S^{G}$, and let $\pi: \widetilde{X} \longrightarrow$ Spec $R$ be the minimal resolution of singularities with exceptional curves $E_{1}, \ldots, E_{n}$. Then

(i) There is a one-one correspondence between 
(a) the exceptional curves $E_{i}$;

(b) the irreducible representations of $G$; and

(c) the indecomposable MCM R-modules.

(ii) (McKay) The dual graph of the desingularization is isomorphic to the McKay quiver after deleting the trivial vertex and replacing pairs of opposed arrows by edges. It is an $A D E$ Coxeter-Dynkin diagram.

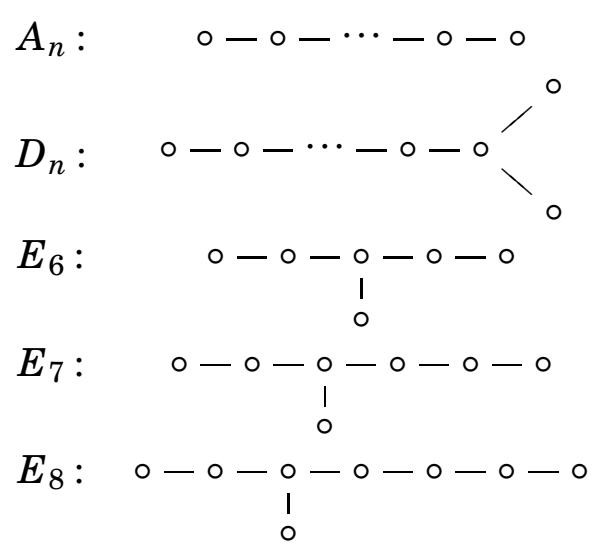

Shortly after McKay's original observation [McK80] of the isomorphism of graphs above, Gonzalez-Sprinberg and Verdier [GSV81] gave, in characteristic zero, a geometric construction linking the representation theory of $G$ and the resolution of singularities $\widetilde{X}$. Later constructions by Artin-Verdier [AV85], Esnault [Esn85], and Knörrer [Knö85] made explicit the correspondences between the exceptional curves $E_{i}$, the indecomposable reflexive $R$-modules, and the irreducible representations of $G$.

The first intimation of a "higher geometric McKay correspondence" appeared in string theory in the mid-1980s. Dixon, Harvey, Vafa, and Witten [DHVW85] observed that for certain $G \subseteq \mathrm{SL}(3, \mathbb{C})$, and a certain crepant resolution $\widetilde{X} \longrightarrow \mathbb{C}^{3} / G$, there is an equality between the Euler characteristic $\chi(\widetilde{X})$ and the number of conjugacy classes (= number of irreducible representations) of $G$. There followed a great deal of work on the existence of crepant resolutions of singularities for quotient singularities of the form $Y / G$, where $Y$ is an arbitrary smooth variety of dimension two or more. Specifically, one can ask for the existence of a crepant resolution $\widetilde{X} \longrightarrow Y / G$ and a derived equivalence between $\widetilde{X}$ and the $G$-equivariant coherent sheaves on $Y$. Let $D_{G}^{b}(Y)$ denote the bounded derived category of the latter.

Such an equivalence was first constructed by Kapranov and Vasserot in the setting of Theorem J.6. In this case, the minimal resolution of singularities $\widetilde{X}$ has an alternative construction, as Nakamura's G-Hilbert scheme Hilb ${ }^{G}\left(\mathbb{C}^{2}\right)[$ [Nak01, IN99]. This is an irreducible component of the subspace of the Hilbert scheme of points in $\mathbb{C}^{2}$ given by the ideal sheaves $\mathscr{I} \subseteq \mathscr{O}_{\mathbb{C}^{2}}$ such that the $\mathscr{O}_{\mathbb{C}^{2}} / \mathscr{I} \cong \mathbb{C}[G]$ as $G$-modules. 
Theorem J.7 (Kapranov-Vasserot [KV00]). Let $G \subset \mathrm{SL}(2, \mathbb{C})$ be a finite group, $S=\mathbb{C}[u, v]$, $R=S^{G}$, and $X=\operatorname{Spec} R$. Set $H=\operatorname{Hilb}^{G}\left(\mathbb{C}^{2}\right)$. Then there is a commutative triangle

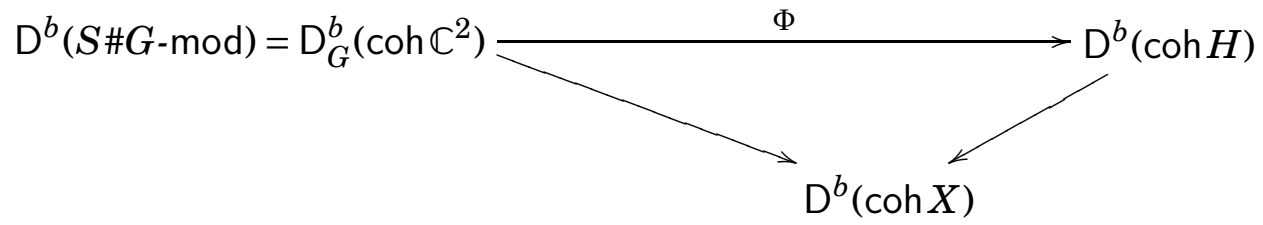

in which $\Phi$ is an equivalence of triangulated categories.

The equivalence $\Phi$ is given by an explicit "equivariant" Fourier-Mukai type functor, $\Phi(-)=$ $\left(\mathbf{R} p_{*} \mathbf{L} q^{*}(-)\right)^{G}$, where $Z \subseteq X \times \mathbb{C}^{2}$ is the incidence variety and $p, q$ are the projections onto the factors.

In dimension greater than two, there is no minimal resolution of singularities. However, Nakamura's $G$-Hilbert scheme is still a candidate for a crepant resolution of singularities in dimension three. Bridgeland, King, and Reid proved the following general result about the $G$-Hilbert scheme.

Theorem J.8 (Bridgeland-King-Reid [BKR01]). Suppose that $Y$ is a smooth and quasiprojective complex variety, and that $G \subseteq$ Aut $Y$ is a finite group of automorphisms such that the quotient $Y / G$ has Gorenstein singularities. Let $H=\operatorname{Hilb}^{G}(Y)$. If

$$
\operatorname{dim}\left(H \times_{Y / G} H\right) \leqslant \operatorname{dim} H+1,
$$

then $H$ is a crepant resolution of singularities of $Y / G$ and there is an equivalence (explicitly given by a Fourier-Mukai functor) of derived categories $\mathrm{D}^{b}\left(\mathrm{Hilb}^{G}(Y)\right) \longrightarrow \mathrm{D}_{G}^{b}(Y)$, where $\mathrm{D}_{G}^{b}(Y)$ is the bounded derived category of G-equivariant coherent sheaves on $Y$.

The assumption on the fiber product $H \times_{Y / G} H$ is automatic if $\operatorname{dim} Y \leqslant 3$, so this result implies a derived McKay correspondence for three-dimensional quotient singularities $\mathbb{C}^{3} / G$ with $G \subset \mathrm{SL}(3, \mathbb{C})$. In particular, such singularities have a crepant resolution, which had been verified on a case-by-case basis using the classification of finite subgroups of SL(3, $\mathbb{C})$. The full details of the correspondences in dimension three are still being worked out [CL09].

In dimension four, the hypothesis on $H \times_{Y / G} H$ need not hold if $H \longrightarrow Y / G$ contracts a divisor to a point. Indeed, we have seen in Example G.6 that some quotients $\mathbb{C}^{4} / G$ have no crepant resolutions of singularities. Furthermore, even when a crepant resolution exists, the $G$-Hilbert scheme may be singular, or non-crepant, or both [Rei02, Example 5.4]. In general, the following conjecture is due to Reid.

Conjecture J.9 (Derived McKay Correspondence Conjecture). For a crepant resolution of singularities $\widetilde{X} \longrightarrow \mathbb{C}^{n} / G$, should one exist, there is an equivalence between $\mathrm{D}^{b}(\mathrm{coh} \widetilde{X})$ and $\mathrm{D}_{G}^{b}\left(\mathbb{C}^{n}\right)$.

Compare with Conjecture $\mathrm{H} .3$ above. The derived McKay correspondence conjecture is known when $G$ preserves a complex symplectic form on $\mathbb{C}^{n}$ [BK04], and when $G$ is abelian [Kaw06].

Notice, for a last comment, that the "resolution" $S \# G \cong \operatorname{End}_{R}(S)$ of Theorem J.2 exists in any dimension for $G \subset \mathrm{GL}(n, k)$ having no non-trivial pseudo-reflections, and delivers a derived equivalence $\mathrm{D}^{b}(S \# G) \simeq \mathrm{D}_{G}^{b}\left(\mathbb{C}^{n}\right)$ by definition. In dimension two, it is even derived equivalent to the "preferred" desingularization $\operatorname{Hilb}^{G}\left(\mathbb{C}^{2}\right)$. As we shall see in the next $\S$, it is 
even in a certain sense "crepant," so represents a potential improvement on the geometric situation.

\section{K. NON-COMmutative CREPANT RESOlutions}

Now I come to the title character of this article. It is an attempt, due to Van den Bergh, to define a concrete algebraic object whose derived category will realize a categorical desingularization in the sense of Definition I.1, and which will also verify Conjecture I.3. The main motivations are Examples H.1 and I.2, and §.

Let $R$ be a commutative ring. Recall from Definitions F.2 and F.3 that a ring $\Lambda$ is an $R$ order if it is finitely generated and MCM as an $R$-module, and is non-singular if gldim $\Lambda_{\mathfrak{p}}=$ $\operatorname{dim} R_{\mathfrak{p}}$ for all $\mathfrak{p} \in \operatorname{Spec} R$. Let us also agree that a module-finite algebra $\Lambda$ over a domain $R$ is birational to $R$ if $\Lambda \otimes_{R} K \cong M_{n}(K)$ for some $n$, where $K$ is the quotient field. If $\Lambda$ is torsionfree as an $R$-module, this is equivalent to asking that $\Lambda \subseteq M_{n}(K)$ and that $\Lambda$ spans $M_{n}(K)$ when scalars are extended to $K$. The terminology is consistent with our determination to identify objects that are Morita equivalent; the birationality of $\Lambda$ should mean that $\Lambda \otimes_{R} K$ is Morita equivalent to $K$, and the only candidates are the matrix rings $M_{n}(K)$.

Here is a provisional definition, to be improved shortly.

Provisional Definition K.1. Let $R$ be a $C M$ normal domain with quotient field $K$. A noncommutative desingularization of $R$ is a non-singular birational $R$-order $\Lambda$.

There is also a natural candidate for a "crepancy" condition.

Definition K.2. Let $R$ be a local ring, and let $\Lambda$ be a module-finite $R$-algebra. Let us say that $\Lambda$ is a symmetric $R$-algebra if $\operatorname{Hom}_{R}(\Lambda, R) \cong \Lambda$ as a $(\Lambda$ - $\Lambda)$-bimodule.

Notice immediately that if $\Lambda$ is a symmetric $R$-algebra, then for any left $\Lambda$-module $M$, there are natural isomorphisms

$$
\operatorname{Hom}_{\Lambda}(M, \Lambda) \cong \operatorname{Hom}_{\Lambda}\left(M, \operatorname{Hom}_{R}(\Lambda, R)\right) \cong \operatorname{Hom}_{R}\left(\Lambda \otimes_{\Lambda} M, R\right) \cong \operatorname{Hom}_{R}(M, R) .
$$

We also have the following direct consequence of Proposition F.4;

Corollary K.3. Let $R$ be a Gorenstein local ring. If $\Lambda$ is a symmetric $R$-order of finite global dimension, then $g l \operatorname{dim} \Lambda=\operatorname{dim} R$. In particular, $\Lambda$ is non-singular.

Notice that this Corollary fails badly for non-Gorenstein $R$; a counterexample is Example P.3 below.

Here finally is the definition [VdB04b].

Definition K.4. Let $(R, \mathfrak{m})$ be a CM local normal domain with quotient field $K$. A noncommutative crepant resolution of $R$ (or of $\operatorname{Spec} R$ ) is a symmetric, birational, $R$-order $\Lambda$ having finite global dimension.

I first observe that the definition is Morita-invariant, i.e. if $\Lambda$ and $\Gamma$ are Morita-equivalent $R$-algebras and $\Lambda$ is a symmetric birational $R$-order of finite global dimension, then so is $\Gamma$. Indeed, global dimension is known to pass across Morita equivalence. Suppose $\Lambda$ is MCM over $R$ and $\Gamma=\operatorname{End}_{\Lambda}(P)$ for some $\Lambda$-progenerator $P$. Since $P$ is a progenerator, $P$ is a direct summand of $\Lambda^{n}$ for some $n$, and it follows that $\Gamma$ is a direct summand of $\operatorname{End}_{\Lambda}\left(\Lambda^{n}\right) \cong M_{n}(\Lambda)$ as an $R$-module. Thus $\Gamma$ is a MCM $R$-module as well. Symmetry is similarly easy to verify. 
Before considering other possible definitions and addressing the examples from previous sections, I point out a connection with the classical theory of orders [AG60, Rei03], following [IR08]. Let $R$ be a domain with quotient field $K$. Recall that a module-finite $R$-algebra $\Lambda$, contained in a finite-dimensional division algebra $D$ over $K$, is called a classical order in $D$ if $\Lambda$ spans $D$ over $K$, and is called maximal in $D$ if it is maximal among classical orders in $D$ with respect to containment. Maximal orders over Dedekind domains have been completely understood for many years; the following facts are well-known.

- Every finite-dimensional division $K$-algebra $D$ contains a unique maximal order $\Delta_{D}$.

- A classical order is maximal if and only if it is Morita equivalent to $\Delta_{D_{1}} \times \cdots \times \Delta_{D_{k}}$ for finite-dimensional division algebras $D_{1}, \ldots, D_{k}$ over $K$.

- A classical order is hereditary, that is, has global dimension at most one, if and only if it is Morita equivalent to a ring of the form $T_{n_{1}}\left(\Delta_{D_{1}}\right) \times \cdots \times T_{n_{k}}\left(\Delta_{D_{k}}\right)$, where $T_{n}(\Delta)$ denotes the subring of $M_{n}(\Delta)$ containing matrices $\left(a_{i j}\right)$ with $a_{i j} \in \operatorname{rad}(\Delta)$ for $i>j$.

With these facts in mind, let $R$ be a complete discrete valuation ring and $\Lambda$ a module-finite $R$-algebra. If $\Lambda$ is a symmetric $R$-algebra of global dimension 1 , then it follows that $\Lambda$ is a maximal order. Indeed, $\Lambda$ is hereditary, so Morita equivalent to $T_{n_{1}}\left(\Delta_{D_{1}}\right) \times \cdots \times T_{n_{k}}\left(\Delta_{D_{k}}\right)$ as above. One can check, however, that $T_{n}(\Delta)$ is symmetric only for $n=1$. Thus $\Lambda$ is maximal.

Now, a classical order $\Lambda$ over a normal domain $R$ is maximal if and only if $\Lambda$ is reflexive as an $R$-module and $\Lambda_{\mathfrak{p}}$ is a maximal order for all primes $\mathfrak{p}$ of height one in $R$ [AG60, 1.5], [Rei03, 11.5]. Combining this with the discussion above gives the following result.

Proposition K.5. Let $R$ be a normal domain with quotient field $K$, and $\Lambda$ a symmetric birational $R$-order of finite global dimension. Then $\Lambda$ is a maximal order.

The connection with the classical theory of (classical) orders gives a structure theorem for symmetric non-singular orders, via the following results of Auslander-Goldman [AG60, Lemma 4.2] and Auslander [Aus86b, Lemma 5.4].

Theorem K.6. Let $R$ be a normal domain with quotient field $K$.

(i) Let $\Lambda$ be a classical order over $R$ in $M_{n}(K)$. Then $\Lambda$ is a maximal order if and only if there exists a finitely generated reflexive $R$-module $M$ such that $\Lambda \cong \operatorname{End}_{R}(M)$.

(ii) Let $M$ be a reflexive $R$-module, and set $\Lambda=\operatorname{End}_{R}(M)$. Then $\Lambda$ is reflexive as an $R$-module and the map $\alpha: \Lambda \longrightarrow \operatorname{Hom}_{R}(\Lambda, R)$ defined by $\alpha(f)(g)=\operatorname{tr}(f g)$, where tr: $\operatorname{End}_{K}\left(K \otimes_{R} M\right) \longrightarrow \operatorname{End}_{R}(M)$ is the usual trace map, is an isomorphism of $(\Lambda-\Lambda)$ bimodules. Hence $\Lambda$ is a symmetric $R$-algebra.

Here are a few definitions which, at least under certain hypotheses, are equivalent to Definition K.4. Part (ii) of the next Proposition is the original definition of a non-commutative crepant resolution [VdB04b, VdB04a]. For that definition, say that $\Lambda$ is homologically homogeneous over the central subring $R$ if it is finitely generated as an $R$-module and every simple $\Lambda$-module has the same projective dimension, equal to $\operatorname{dim} R$ [BH84, BHM82]. This condition seems first to have been introduced by Vasconcelos [Vas73] under the name "moderated algebras." If $R$ is equidimensional, it is equivalent to asking that for every $\mathfrak{p} \in \operatorname{Spec} R$ the localization $\Lambda_{\mathfrak{p}}$ is MCM as an $R_{\mathfrak{p}}$-module and gldim $\Lambda_{\mathfrak{p}}=\operatorname{dim} R_{\mathfrak{p}}$ [BHM83].

Proposition K.7. Let $R$ be a Gorenstein local normal domain and let $\Lambda$ be a module-finite $R$-algebra. Then the following sets of conditions on $\Lambda$ are equivalent.

(i) $\Lambda$ is a symmetric birational $R$-order and has finite global dimension. 
(ii) $\Lambda \cong \operatorname{End}_{R}(M)$ for some reflexive $R$-module $M$, and $\Lambda$ is homologically homogeneous.

(iii) $\Lambda \cong \operatorname{End}_{R}(M)$ for some reflexive $R$-module $M, \Lambda$ is $M C M$ as an $R$-module, and $\operatorname{gldim} \Lambda<\infty$.

Proof. Assume first that $\Lambda$ satisfies (i). Then $\Lambda$ is MCM over $R$ by definition, and this localizes well. By Theorem K.6.(i), $\Lambda$ is an endomorphism ring of a reflexive module $M$, and by Corollary K.3 $\Lambda$ is non-singular, giving (ii). Clearly (ii) implies (iii). Finally, if $\Lambda \cong \operatorname{End}_{R}(M)$ for a reflexive $R$-module $M$, then $\Lambda$ is birational to $R$, and is symmetric by Theorem K.6.ii).

The implication (iii) $\Longrightarrow$ (ii) fails if $R$ is not Gorenstein. Again see Example P.3 below.

Now it is clear that Auslander's Theorem J.2 proves that, for any $n \geqslant 2$ and any finite group $G \subset \operatorname{SL}(n, k)$ with order invertible in $k$, the ring of invariants $R=k \llbracket x_{1}, \ldots, x_{n} \rrbracket^{G}$ has a non-commutative crepant resolution. Namely, with $S$ denoting the power series ring, the endomorphism ring $\operatorname{End}_{R}(S)$ has finite global dimension and, since $S$ is a MCM $R$-module, is an $R$-order. Thus $\operatorname{End}_{R}(S) \cong S \# G$ is a non-commutative crepant resolution. One can also prove directly that the twisted group ring $S \# G$ is symmetric over $R$.

Similarly, the three-dimensional ordinary double point in Example I.2 admits the noncommutative crepant resolution $\Lambda=\operatorname{End}_{R}(R \oplus I)$, which is derived equivalent to the resolutions of singularities $Y$ and $Y^{\prime}$.

For the next equivalent definition we need the notion of a $\mathrm{d}$-Calabi-Yau algebra. There are a few approaches to topics with this name. I follow [IR08, Bra07]; see also [Gin06, Boc09]. I will always assume that the base ring is local, which eases the exposition considerably.

Definition K.8. Let $R$ be a local ring and let $\Lambda$ be a module-finite $R$-algebra. Write $D(-)=$ $\operatorname{Hom}_{R}(-, E)$ for Matlis duality over $R$, where $E$ is the injective hull of the residue field. Say that $\Lambda$ is $d$-Calabi-Yau $(d-C Y)$ if there is a functorial isomorphism

$$
\operatorname{Hom}_{\mathrm{D}(\Lambda \text {-Mod })}(X, Y[d]) \cong D \operatorname{Hom}_{\mathrm{D}(\Lambda \text {-Mod })}(Y, X)
$$

for all $X$ and $Y$ in $D^{b}(\Lambda$-fl), the bounded derived category of finite-length $\Lambda$-modules. Similarly, $\Lambda$ is $d-C Y^{-}$if an isomorphism as above holds for all $X$ in $D^{b}(\Lambda-f l)$ and all $Y$ in $\mathrm{K}^{b}(\operatorname{add} \Lambda)$.

These definitions are perhaps a bit much to swallow all at once. Here are some basic facts about the Calabi-Yau conditions. Let $R$ be a local ring and $\Lambda$ a module-finite $R$-algebra. Then $\Lambda$ is $n$-CY for some integer $n$ if and only if $\Lambda$ is $n-\mathrm{CY}^{-}$and has finite global dimension. Indeed, if gldim $\Lambda<\infty$ then $\mathrm{D}^{b}(\Lambda-\mathrm{fl}) \subset \mathrm{D}^{b}(\Lambda$-mod $)=\mathrm{K}^{b}(\operatorname{add} \Lambda)$. The "only if" part is proved by completing and considering the finite-length $\Lambda$-module $Y / \operatorname{rad}(\Lambda)^{n} Y$ for $Y$ in $\mathrm{K}^{b}(\operatorname{add} \Lambda)$.

Calabi-Yau algebras are best-behaved when $R$ is Gorenstein. In that case [IR08, Theorem 3.2], if $\Lambda$ is $n$-CY or $n-\mathrm{CY}^{-}$for some $n$, then $n=\operatorname{dim} R$. Furthermore, $\Lambda$ is $d$-CY- if and only if $\Lambda$ is a symmetric $R$-order. (This is one point where life is easier because $R$ is local. Iyama and Reiten give an example, which they credit to J. Miyachi, of a $d$-CY ${ }^{-}$algebra over a nonlocal Gorenstein ring which is not symmetric, even though $R \longrightarrow \Lambda$ is injective. It is locally symmetric.) More precisely, the following equivalent conditions hold.

Proposition K.9 ([IR08]). Let $(R, \mathfrak{m}, k)$ be a Gorenstein local ring with $\operatorname{dim} R=d$, and let $\Lambda$ be a module-finite $R$-algebra. The following are equivalent for any integer $n$.

(i) $\Lambda$ is $n-C Y^{-}$. 
(ii) As functors on $\Lambda$-fl, $\operatorname{Ext}_{\Lambda}^{n}(-, \Lambda)$ is isomorphic to the Matlis duality functor $D(-)=$ $\operatorname{Hom}_{R}(-, E)$, and $\operatorname{Ext}_{\Lambda}^{i}(-, \Lambda)=0$ for $i \neq n$.

(iii) $\mathbf{R H o m}_{R}(\Lambda, R) \cong \Lambda[n-d]$ in the bounded derived category of $(\Lambda-\Lambda)$-bimodules.

(iv) $\Lambda$ is a CM R-module of dimension $n$ and $\operatorname{Ext}_{R}^{d-n}(\Lambda, R) \cong \Lambda$ as $(\Lambda-\Lambda)$-bimodules.

In particular, a birational module-finite algebra $\Lambda$ is $d$-CY if and only if it is symmetric and has finite global dimension.

Of course the value of a definition, even one as motivated as this one has been, is in the theorems. Here is the main result of [VdB04b].

Theorem K.10. Let $R$ be a Gorenstein normal $\mathbb{C}$-algebra, $X=\operatorname{Spec} R$, and $\pi: \widetilde{X} \longrightarrow X a$ crepant resolution of singularities. Assume that the fibers of $\pi$ have dimension at most one. Then there exists a $M C M R$-module $M$ such that the endomorphism ring $\Lambda=\operatorname{End}_{R}(M)$ is homologically homogeneous. In particular, $\Lambda$ is a non-commutative crepant resolution of $R$. Furthermore, $\widetilde{X}$ and $\Lambda$ are derived equivalent: $\mathrm{D}^{b}(\operatorname{coh} \widetilde{X}) \simeq \mathrm{D}^{b}(\Lambda$-mod).

Here is a sketch of the proof of Theorem K.10, We know that existence of a crepant resolution implies that $X$ has rational singularities. Let $\mathscr{L}$ be an ample line bundle on the smooth variety $\widetilde{X}$ generated by global sections. Then by the hypothesis on the fibers of $\pi$ ([BVdB03, 4.2.4]), $\mathscr{O}_{\tilde{X}} \oplus \mathscr{L}$ generates $\mathrm{D}(\mathrm{Qch} \widetilde{X})$, that is, if $\mathscr{N}$ in $\mathrm{D}(\mathrm{Q} \operatorname{ch} \widetilde{X})$ satisfies $\operatorname{Hom}_{\mathrm{D}(Q \mathrm{Qch} \tilde{X})}\left(\mathscr{O}_{\tilde{X}} \oplus \mathscr{L}, \mathscr{N}[i]\right)=0$ for $i \neq 0$, then $\mathscr{N}=0$ (see the discussion after Definition D.2). Take an extension $0 \longrightarrow \mathscr{O}_{\tilde{X}}^{r} \longrightarrow \mathscr{M}^{\prime} \longrightarrow \mathscr{L} \longrightarrow 0$ corresponding to a set of $r$ generators for $\operatorname{Ext}_{\mathscr{O}_{\tilde{X}}}^{1}\left(\mathscr{L}, \mathscr{O}_{\tilde{X}}\right)$ as an $R$-module. Set $\mathscr{M}=\mathscr{M}^{\prime} \oplus \mathscr{O}_{\tilde{X}}$. Then $\mathscr{M}$ also generates $\mathrm{D}(\mathrm{Q} c h \widetilde{X})$. One can show that $\operatorname{Ext}_{\mathscr{O}_{\tilde{X}}}^{i}(\mathscr{M}, \mathscr{M})=0$ for $i>0$ (this takes a good bit of work). Thus $\mathscr{M}$ is a tilting bundle on $\widetilde{X}$. Set $\Lambda=\operatorname{End}_{\mathscr{O}_{\tilde{X}}}(\mathscr{M})$; then the vanishing of the derived pushforwards $\mathbf{R}^{i} \pi_{*} \mathscr{E} n d_{\mathscr{O}_{\tilde{X}}}(\mathscr{M})=\operatorname{Ext}_{\mathscr{O}_{\tilde{X}}}^{i}(\mathscr{M}, \mathscr{M})$ implies that $\Lambda \cong \operatorname{End}_{R}(M)$, where $M=\Gamma(\widetilde{X}, \mathscr{M})$. The proofs that $\Lambda$ and $M$ are both MCM are more involved.

Van den Bergh also proves a result converse to Theorem K.10, constructing a geometric crepant resolution $\pi: \widetilde{X} \longrightarrow \operatorname{Spec} R$ from a non-commutative one under certain assumptions [VdB04a, §6]. The method is roughly as follows: let $\Lambda$ be a non-commutative crepant resolution of $R$, and take for $\widetilde{X}$ a moduli space of certain stable representations of $\Lambda$. Then he proves that if $\operatorname{dim}\left(\widetilde{X} \times_{\operatorname{Spec} R} \widetilde{X}\right) \leqslant \operatorname{dim} R+1$, then $\widetilde{X} \longrightarrow \operatorname{Spec} R$ is a crepant resolution and there is an equivalence of derived categories $\mathrm{D}^{b}(\operatorname{coh} \widetilde{X}) \simeq \mathrm{D}^{b}(\Lambda$-mod). Observe that the hypothesis is exactly similar to that of Theorem J.8. In particular, the hypothesis holds if $\operatorname{dim} R \leqslant 3$, giving the following theorem.

Theorem K.11. Let $R$ be a three-dimensional Gorenstein normal $\mathbb{C}$-algebra with terminal singularities.

(i) There is a non-commutative crepant resolution of $R$ if and only if $X=\operatorname{Spec} R$ has a crepant resolution of singularities.

(ii) All crepant resolutions of $R$-geometric as well as non-commutative-are derived equivalent.

The second statement verifies Conjecture $\mathrm{H} .3$ of Bondal and Orlov in this case. Iyama and Reiten [IR08] have recently shown that, even without the assumption on the singularities of $R$ being terminal, all non-commutative crepant resolutions of $R$ are derived equivalent. 
Even more recently, Iyama and Wemyss [IW11] have announced a sufficient criterion for the existence of a derived equivalence between the noncommutative crepant resolutions of $R$. When $d \leqslant 3$ this criterion is always satisfied, recovering the Iyama-Reiten result.

Van den Bergh suggests the following extension of Theorem K.11(ii).

Conjecture K.12 (Van den Bergh). Let $R$ be a Gorenstein normal $\mathbb{C}$-algebra and $X=\operatorname{Spec} R$. Then all crepant resolutions of $R$-geometric as well as non-commutative-are derived equivalent.

\section{EXAMPLE: NORMALIZATION}

The appearance of endomorphism rings as ersatz resolutions of singularities may initially be unsettling. It does, however, have a precedent. One can think of the normalization $\bar{R}$ of an integral domain $R$, i.e. the integral closure in its quotient field, as a partial resolution of singularities, one that is especially tractable since it does not leave the category of noetherian rings. This result of Grauert and Remmert [GR71, GR84] interprets the normalization as an endomorphism ring.

Theorem L.1 (Grauert-Remmert). Let $R$ be an integral domain and $I$ a non-zero integrally closed ideal of $R$ such that $R_{\mathfrak{p}}$ is normal for every $\mathfrak{p} \not \supset I$. Then the following are equivalent.

(i) $R$ is normal;

(ii) For all non-zero fractional ideals $J$ of $R, \operatorname{Hom}_{R}(J, J)=R$;

(iii) For all non-zero ideals $J$ of $R, \operatorname{Hom}_{R}(J, J)=R$;

(iv) $\operatorname{Hom}_{R}(I, I)=R$.

For any fractional ideal $J$, the containments $R \subseteq \operatorname{Hom}_{R}(J, J) \subseteq \bar{R}$ always hold. The latter inclusion sends $\varphi: J \longrightarrow J$ to the fraction $\varphi(r) / r$ for any fixed non-zerodivisor $r \in J$; this is well-defined. In particular, $\operatorname{Hom}_{R}(J, J)$ is a commutative (!) ring.

Theorem L.1 was used by de Jong [dJ98] to give an algorithm for computing the normalization $\bar{R}$ of an affine domain over a perfect field, or slightly more generally. Let $R$ be a local domain such that its normalization $\bar{R}$ is a finitely generated $R$-module; equivalently, the completion $\widehat{R}$ is reduced. One needs to determine a non-zero integrally closed ideal $I$ such that $V(I)$ contains the non-normal locus of $R$. If $R$ is affine over a perfect field, then the Jacobian criterion implies that the radical of the Jacobian ideal will work; there are other choices in other cases. Set $R^{\prime}=\operatorname{Hom}_{R}(I, I)$. If $R^{\prime}=R$, then $R$ is normal, so stop. Otherwise, replace $R$ by $R^{\prime}$ and repeat. The algorithm has been refined and extended since [DdJGP99, GLS10].

It follows from Serre's criterion for normality that if $R$ is the coordinate ring of an irreducible curve singularity, then the normalization $\bar{R}$ is regular, whence is the coordinate ring of a resolution of singularities of $\operatorname{Spec} R$. Thus in this situation, desingularization can be achieved as an iterated endomorphism ring. In fact, as long as $R$ is affine over a perfect field, one actually has $\bar{R}=\operatorname{Hom}_{R}(\bar{R}, \bar{R})$, a single endomorphism ring of a finitely generated module giving resolution of singularities.

\section{MCM ENDOMORPHISM RINGS}

The requirement that a non-commutative crepant resolution of singularities should be an order, i.e. a MCM module, raises a basic question: Does the depth of $\operatorname{Hom}_{R}(M, M)$ depend in 
any predictable way on the depth of $M$ ? The short answer is No. In this $\S$ we look at some examples.

First, observe that there is at least a lower bound on the depth of a Hom module: If $R$ is any local ring and $M, N$ are finitely generated modules with $\operatorname{depth} N \geqslant 2$, then $\operatorname{Hom}_{R}(M, N)$ has depth $\geqslant 2$ as well. Indeed, applying $\operatorname{Hom}_{R}(-, N)$ to a free presentation of $M$ displays $\operatorname{Hom}_{R}(M, N)$ as the kernel of a map between direct sums of copies of $N$, so the Depth Lemma gives the conclusion. That's about the end of the good news.

Next notice that the depth of $\operatorname{Hom}_{R}(M, M)$ can be strictly greater than that of $M$. Indeed, let $R$ be a CM normal domain and let $J$ be any non-zero ideal of $R$. Then $\operatorname{Hom}_{R}(J, J)=R$ by Theorem L.1, even though $\operatorname{depth}_{R} J$ can take any value between 1 (if, say, $J$ is a maximal ideal) and $d$ (if for example $J$ is principal). Furthermore, $R$ can be taken to be Gorenstein, or even a hypersurface ring, so finding a class of rings that avoids this problem seems hopeless.

One might hope at least that if $M$ is $\operatorname{MCM}$ then $\operatorname{Hom}_{R}(M, M)$ is MCM as well. This question was raised by Vasconcelos [Vas68] for $R$ a Gorenstein local ring. It also has a negative answer, though it is at least harder. A counterexample is given by Jinnah [Jin75], based on [Hoc73, Example 5.9].

Example M.1. Let $k$ be a field and set $A=k[x, y, z] /\left(x^{3}+y^{3}+z^{3}\right), B=k[u, v]$. Let $R$ be the Segre product of $A$ and $B$, the graded ring defined by $R_{n}=A_{n} \otimes_{k} B_{n}$. Then $R$ is the subring of $A[u, v]$ generated by $x u, x v, y u, y v, z u, z v$, a three-dimensional normal domain of depth 2 . The ideal $I=v A[u, v] \cap R$ has depth 3 over $R$.

Write $R$ as a quotient of a graded complete intersection ring $S$ of dimension 3 . Then $I$ has depth 3 over $S$ as well, but $\operatorname{Hom}_{S}(I, I)=\operatorname{Hom}_{R}(I, I)=R$ has depth two as an $S$-module. Localizing $S$ at its irrelevant ideal gives a local example.

Here is a useful characterization of the depth of $\operatorname{Hom}_{R}(M, N)$.

Lemma M.2 ([Iya07b, Dao10]). Let $R$ be a $C M$ local ring and let $M$ and $N$ be finitely generated $R$-modules. Fix $n \geqslant 2$ and consider the following properties.

(i) $\operatorname{Hom}_{R}(M, N)$ satisfies $\left(S_{n+1}\right)$; and

(ii) $\operatorname{Ext}_{R}^{i}(M, N)=0$ for $i=1, \ldots, n-1$.

If $M$ is locally free in codimension $n$ and $N$ satisfies $\left(S_{n}\right)$, then (ii) $\Longrightarrow$ (iil). If $N$ satisfies $\left(S_{n+1}\right)$ then (ii) $\Longrightarrow($ i $)$.

Since this result is used in some later sections, I'll sketch the proof. First assume that $M$ is locally free in codimension $n$ and that $N$ satisfies $\left(S_{n}\right)$. If $n \geqslant \operatorname{dim} R$ then $M$ is free and there is nothing to prove, so one may localize at a prime ideal of height $n+1$ to assume by induction that $M$ is locally free on the punctured spectrum, and so $\operatorname{Ext}_{R}^{i}(M, N)$ has finite length for $i \geqslant 1$. Take a free resolution $P$. of $M$ and consider the first $n$ terms of the complex $\operatorname{Hom}_{R}(P ., N)$. Since the cohomologies of this complex, namely $\operatorname{Ext}_{R}^{i}(M, N)$ for $i=1, \ldots, n-1$, all have finite length, $\operatorname{Hom}_{R}(P ., N)$ is exact by the Acyclicity Lemma [BH93, Ex. 1.4.23]. For the second statement, take once again the free resolution $P$. of $M$ and consider the first $n$ terms of $\operatorname{Hom}_{R}\left(P_{.}, N\right)$, which form an exact sequence by the assumption. The Depth Lemma then implies $\operatorname{depth} \operatorname{Hom}_{R}(M, N)_{\mathfrak{p}} \geqslant \min \left\{n+1, \operatorname{depth} N_{\mathfrak{p}}\right\}$ for every $\mathfrak{p} \in \operatorname{Spec} R$, which gives the conclusion.

The homological consequences of Lemma M.2 are even stronger than is immediately apparent. To describe these, recall that module $N$ over a commutative ring $R$ is called Tor-rigid 
if whenever $\operatorname{Tor}_{i}^{R}(M, N)=0$ for some $i \geqslant 0$ and some finitely generated $R$-module $M$, necessarily $\operatorname{Tor}_{j}^{R}(M, N)=0$ for all $j \geqslant i$. Deciding whether a given module is Tor-rigid is generally a delicate problem, as Dao observes [Dao10]. The following result of Jothilingam [Jot75] (see also [Jor08]) gives a very useful necessary condition.

Proposition M.3 (Jothilingam). Let $R$ be a local ring and let $M, N$ be finitely generated $R$ modules. Assume that $N$ is Tor-rigid. If $\operatorname{Ext}_{R}^{1}(M, N)=0$, then the natural map $\Phi_{M, N}: M^{*} \otimes_{R}$ $N \longrightarrow \operatorname{Hom}_{R}(M, N)$ is an isomorphism. In particular, if $\operatorname{Ext}_{R}^{1}(N, N)=0$ then $N$ is free.

It follows immediately that if $R$ is a local ring satisfying $\left(R_{2}\right)$ and $\left(S_{3}\right)$, and $M$ is a reflexive $R$-module with a non-free direct summand which is Tor-rigid, then $\Lambda=\operatorname{End}_{R}(M)$ is not MCM, whence is not a non-commutative crepant resolution. Indeed, let $N$ be a Tor-rigid summand of $M$ which is not free. Then $N$ is reflexive, so satisfies $\left(S_{2}\right)$, and is free in codimension two as $R$ is regular on that locus. Moreover, $\operatorname{Hom}_{R}(N, N)$ is a direct summand of $\operatorname{Hom}_{R}(M, M)$. If $\operatorname{Hom}_{R}(M, M)$ were MCM, then $\operatorname{Hom}_{R}(N, N)$ would also be, so would satisfy $\left(S_{3}\right)$. But then $\operatorname{Ext}_{R}^{1}(N, N)=0$ by Lemma M.2, contradicting Proposition M.3.

It is now easy to bolster Example M.1 by constructing, over any CM local ring $(R, \mathfrak{m}, k)$ of dimension 3 or more, a MCM module $M$ such that $\operatorname{Hom}_{R}(M, M)$ is not $\mathrm{MCM}^{2}$. Indeed, take $M$ to be a high enough syzygy of the residue field $k$; since $k$ is Tor-rigid, the same is true of $M$, and it is locally free on the punctured spectrum. By Lemma M.2 and Proposition M.3, then, $\operatorname{Hom}_{R}(M, M)$ has depth at most 2 .

From Proposition M.3 and progress on understanding Tor-rigid modules over hypersurface rings, Dao derives the next theorem, which identifies obstructions to the existence of non-commutative crepant resolutions.

Theorem M.4 ([Da008, Dao10, Dao11]). Let $R=S /(f)$ be a local hypersurface ring with $S$ an equicharacteristic or unramified regular local ring and $f \in S$ a non-zero non-unit. Assume that $R$ is regular in codimension two.

(i) If $\operatorname{dim} R=3$ and $R$ is $\mathbb{Q}$-factorial, then every finitely generated $R$-module is Tor-rigid, so $R$ admits no non-commutative crepant resolution.

(ii) If $R$ has an isolated singularity and $\operatorname{dim} R$ is an even number greater than 3 , then $\operatorname{Hom}_{R}(M, M)$ satisfies $\left(S_{3}\right)$ only if $M$ is free, so $R$ admits no non-commutative crepant resolution.

Recall from Example G.5 that the isolated hypersurface singularity defined by $x_{0}^{r}+x_{1}^{d}+$ $\cdots+x_{d}^{d}=0$ has a crepant resolution of singularities only if $r \equiv 0$ or 1 modulo $d$. Part (iil) of Dao's theorem thus implies that the extension of Van den Bergh's Theorem K.11(i) to higher dimensions has a negative answer, at least without some further assumptions.

Example M.5 ([Dao10, Example 3.6], [BIKR08, §2]). Theorem M.4 allows some progress toward deciding which of the three-dimensional ADE singularities (see (G.1)) have noncommutative crepant resolutions. Let $k$ be an algebraically closed field of characteristic zero. The 3-dimensional versions of $\left(A_{2 \ell}\right),\left(E_{6}\right)$, and $\left(E_{8}\right)$ are factorial, so do not admit a non-commutative crepant resolution at all.

Let $R=k \llbracket x, y, z, w \rrbracket /\left(x y+z^{2}-w^{2 \ell+2}\right)$, an $\left(A_{2 \ell+1}\right)$ singularity, with $\ell \geqslant 1$. (Observe that the case $\ell=0$ is the ordinary double point of Example H.1.) Then I claim that $R$ has a

\footnotetext{
${ }^{2}$ I'm grateful to Hailong Dao for pointing this out to me.
} 
non-commutative crepant resolution $\Lambda=\operatorname{End}_{R}(M)$ in which $M$ is MCM. Indeed, the indecomposable MCM $R$-modules are completely known [Yos90, Example 5.12]; they are the free module $R$, the ideal $I=\left(x, z+w^{\ell+1}\right)$, the dual ideal $I^{*}=\left(y, z-w^{\ell+1}\right)$, and $\ell$ indecomposables $M_{1}, \ldots, M_{\ell}$ of rank two.

Each $M_{i}$ is its own Auslander-Reiten translate, $\tau M_{i} \cong M_{i}$, so in particular $\operatorname{Ext}_{R}^{1}\left(M_{i}, M_{i}\right) \neq$ 0 for $i=1, \ldots, \ell$. By Lemma M.2, no $M_{i}$ can be a constituent in a non-commutative crepant resolution. On the other hand, $I$ and $I^{*}$ satisfy $\operatorname{Hom}_{R}(I, I) \cong \operatorname{Hom}_{R}\left(I^{*}, I^{*}\right) \cong R$ by Theorem L.1. Thus at least $\operatorname{End}_{R}(R \oplus I)$ and $\operatorname{End}_{R}\left(R \oplus I^{*}\right)$ are symmetric $R$-orders; it will follow from the results in the next $\S$ that since $R \oplus I$ and $R \oplus I^{*}$ are cluster tilting modules (Theorem N.5), the endomorphism rings have global dimension equal to 3 , so are noncommutative crepant resolutions.

\section{N. Global Dimension of Endomorphism RINGS}

The tendency for endomorphism rings to have finite global dimension was first observed by Auslander [Aus71, §III.3]. Recall that $\Lambda$ is an artin algebra if the center of $\Lambda$ is a commutative artin ring and $\Lambda$ is a finitely generated module over its center.

Theorem N.1 (Auslander). Let $\Lambda$ be an artin algebra with radical $\mathfrak{r}$ and assume that $\mathfrak{r}^{n}=$ $0, \mathfrak{r}^{n-1} \neq 0$. Set $M=\bigoplus_{i=0}^{n} \Lambda / \mathfrak{r}^{i}$. Then $\Gamma=\operatorname{End}_{\Lambda}(M)$ is a coherent artin algebra of global dimension at most $n+1$.

Based on this result Auslander was led to define the representation dimension of an artin algebra $\Lambda$ as the least value of gldim $\operatorname{End}_{\Lambda}(M)$ as $M$ runs through all finitely generated $\Lambda$ modules which are generators-cogenerators for $\Lambda$, that is, $M$ contains as direct summands all indecomposable projective and injective $\Lambda$-modules. Observe that Theorem N.1 does not prove finiteness of the representation dimension; while $M$ has a non-zero free direct summand, it need not be a cogenerator unless $\Lambda$ is self-injective.

Auslander proved in [Aus71] that repdim $\Lambda \leqslant 2$ if and only if $\Lambda$ has finite representation type (see $\S \bar{P}$ ), but it was not until 2003 that Rouquier constructed the first examples with representation dimension greater than 3 [Rou06b]. Incidentally, Rouquier's proof uses the notion of the dimension [BVdB03, Rou08] of the derived category $\mathrm{D}^{b}(\Lambda$-mod). The dimension of a triangulated category is a measure of how many steps are required to obtain it starting from a single object and inductively taking the closure under shifts, direct sums and summands, and distinguished triangles. Rouquier proved that if $\Lambda$ is a finite-dimensional algebra over a perfect field $k$, then $\operatorname{dim} D^{b}(\Lambda$-mod $) \leqslant \operatorname{repdim} \Lambda$.

Iyama showed in 2003 [Iya03] that the representation dimension of a finite-dimensional algebra is always finite. He also extended the definition of representation dimension to CM local rings of positive Krull dimension.

Definition N.2. Let $R$ be a complete CM local ring with canonical module $\omega$. Set

$$
\operatorname{repdim} R=\inf _{M}\left\{\operatorname{gldim} \operatorname{End}_{R}(R \oplus \omega \oplus M)\right\},
$$

where the infimum is taken over all MCM $R$-modules $M$.

Iyama's techniques involved maximal n-orthogonal modules, now called cluster tilting modules [Iya07b]. Here I will not say anything about cluster algebras or cluster categories; see [BM06] for an exposition. Here is a direct definition of cluster tilting modules [BIKR08]. 
Definition N.3. Let $R$ be a CM local ring and $M$ a MCM $R$-module. Fix $n \geqslant 1$.

(i) Set

$$
M^{\perp_{n}}=\left\{X \mid X \text { is MCM and } \operatorname{Ext}_{R}^{i}(M, X)=0 \text { for } 1 \leqslant i \leqslant n\right\}
$$

and symmetrically

$$
{ }^{\perp_{n}} M=\left\{X \mid X \text { is MCM and } \operatorname{Ext}_{R}^{i}(X, M)=0 \text { for } 1 \leqslant i \leqslant n\right\} .
$$

(ii) Say that $M$ is cluster tilting if

$$
M^{\perp_{1}}=\operatorname{add} M={ }^{\perp_{1}} M .
$$

There are some isolated results about cluster tilting in small dimension. For one example, [BIKR08] constructs and classifies cluster tilting modules for the one-dimensional ADE hypersurface singularities. When $R$ is two-dimensional and Gorenstein, the AuslanderReiten translate $\tau$ is the identity, so $\operatorname{Ext}_{R}^{1}(M, M)$ is never zero for MCM $M$; this rules out cluster tilting in this case.

To describe the connection between cluster tilting modules and non-commutative crepant resolutions, let's consider the following theorem of Dao-Huneke [DH10, Theorem 3.2].

Theorem N.4. Let $R$ be a CM local ring of dimension $d \geqslant 3$. Let $M$ be a MCM $R$-module with a non-zero free direct summand, and set $\Lambda=\operatorname{End}_{R}(M)$. Assume that $\Lambda$ is $M C M$ as an $R$-module. Consider the following conditions.

(i) $M^{\perp_{d-2}}=\operatorname{add} M$.

(ii) There exists an integer $n$ with $1 \leqslant n \leqslant d-2$ such that $M^{\perp_{n}}=$ add $M$.

(iii) $\operatorname{gldim} \Lambda \leqslant d$.

(iv) $\operatorname{gldim} \Lambda=d$.

Then (ii) $\Longrightarrow$ (iii) $\Longrightarrow$ (iii) $\Longleftrightarrow$ (iv). If $R$ has an isolated singularity, then all four are equivalent.

The main assertion here is (ii) $\Longrightarrow$ (iii). Everything else is relatively straightforward or follows from Lemma M.2. To prove (ii) $\Longrightarrow$ (iii), Dao and Huneke use Proposition A.4 to get, for any $R$-module $N$ satisfying $\left(S_{2}\right)$, a long exact sequence

$$
\sigma: \quad \cdots \longrightarrow M^{n_{j+1}} \longrightarrow M^{n_{j}} \longrightarrow \cdots \longrightarrow M^{n_{0}} \longrightarrow N \longrightarrow 0
$$

such that $\operatorname{Hom}_{R}(M, \sigma)$ is exact. Let $N_{j}$ be the kernel at the $j^{\text {th }}$ spot; then one shows by induction on $j$ that $\operatorname{Ext}_{R}^{1}\left(M, N_{j}\right) \subseteq \operatorname{Ext}_{R}^{1}(M, M)^{n_{j+1}}$, so that $N_{d-2} \in M^{\perp_{d-2}}=\operatorname{add} M$. It follows that $\operatorname{Hom}_{R}\left(M, N_{d-2}\right)$ is $\Lambda$-projective. Thus every $\Lambda$-module of the form $\operatorname{Hom}_{R}(M, N)$ has projective dimension at most $d-2$, so that gldim $\Lambda \leqslant d$.

As a corollary of Theorem N.4, Dao and Huneke obtained another proof of the following result of Iyama, which nicely encapsulates the significance of cluster tilting modules to noncommutative crepant resolutions.

Theorem N.5 (Iyama [Iya07a, Theorem 5.2.1]). Let $R$ be a CM local ring of dimension $d \geqslant 3$ and with canonical module $\omega$. Assume that $R$ has an isolated singularity. Let $M$ be a MCM $R$-module and set $\Lambda=\operatorname{End}_{R}(M)$. The following conditions are equivalent.

(i) $M$ contains $R$ and $\omega$ as direct summands, $\Lambda$ is $M C M$, and $\operatorname{gldim} \Lambda=d$.

(ii) $M^{\perp_{d-2}}=\operatorname{add} M={ }^{\perp_{d-2}} M$. 
In particular, if $d=3$ and $R$ is a Gorenstein isolated singularity, then a MCM $R$-module $M$ gives a non-commutative crepant resolution if and only if it is a cluster tilting module.

For dimension 3, this result gives a very clear picture of the landscape of non-commutative crepant resolutions. In higher dimension, however, the assumption of isolated singularity becomes more restrictive. Moreover, as Dao and Huneke observe, for $d \geqslant 4$ the condition add $M=M^{\perp_{d-2}}$ rules out a large class of examples. Specifically, if $\operatorname{Ext}_{R}^{2}(M, M)=0$ for a MCM module $M$ over a complete intersection ring $R$, then $M$ is necessarily free, since one can complete and lift $M$ to a regular local ring [ADS93].

Back in dimension 3, one can obtain even stronger results, and address possible extensions of Theorem K.11(ii), by imposing geometric hypotheses. Recall that a cDV sin-

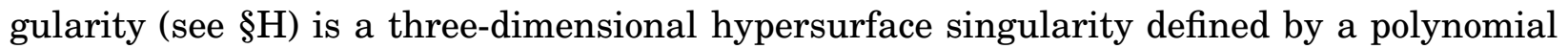
$f(x, y, z)+\operatorname{tg}(x, y, z, t)$, where $f$ is $\mathrm{ADE}$ and $g$ is arbitrary. A cDV singularity is called $c A_{n}$ if the generic hyperplane section is a surface singularity of type $\left(A_{n}\right)$.

Theorem N.6 ([BIKR08, Theorem 5.5]). Let $(R, \mathfrak{m})$ be a local isolated $c D V$ singularity. Then Spec $R$ has a crepant resolution of singularities if and only if $R$ has a non-commutative crepant resolution, and these both occur if and only if there is a cluster tilting module in the stable category $\underline{C M}(R)$. If $R$ is a $c A_{n}$ singularity defined by $g(x, y)+z t$, then these are equivalent to the number of irreducible power series in a prime decomposition of $g(x, y)$ being $n+1$.

\section{O. RATIONAL SINGULARITIES}

As we saw in Proposition G.4, GR Vanishing implies that any complex algebraic variety with a crepant resolution of singularities has rational singularities. Furthermore, the idea of a categorical, or non-commutative, desingularization is really only well-behaved for rational singularities. It would therefore be most satisfactory if existence of a non-commutative crepant resolution - a symmetric birational order of finite global dimension-implied rational singularities. This is true by work of Stafford and Van den Bergh [SVdB08]. Their result is somewhat more general. Recall from the discussion preceding Proposition K.7 that $\Lambda$ is homologically homogeneous if every simple $\Lambda$-module has the same projective dimension.

Theorem 0.1 (Stafford-Van den Bergh). Let $k$ be an algebraically closed field of characteristic zero, and let $\Lambda$ be a prime affine $k$-algebra which is finitely generated as a module over its center $R$. If $\Lambda$ is homologically homogeneous then the center $R$ has rational singularities. In particular, if $R$ is a Gorenstein normal affine domain and has a non-commutative crepant resolution of singularities, then it has rational singularities.

Van den Bergh gave a proof of the final sentence in case $R$ is graded in [VdB04a, Proposition 3.3]. (This argument in the published version of [VdB04a] is not quite correct; see the updated version online for a corrected proof.)

Here are a few comments on the proof, only in the case where $\Lambda$ is a non-commutative crepant resolution of its Gorenstein center $R$, so is symmetric, birational and of finite global dimension. The first step is a criterion for rational singularities, which is an algebraicization of the criterion $\pi_{*} \omega_{\tilde{X}}=\omega_{X}$ of Proposition G.4.

Lemma 0.2. Let $R$ be a CM normal affine $k$-algebra, where $k$ is an algebraically closed field of characteristic zero. Let $K$ be the quotient field of $R$ and let $\omega_{R}$ be the canonical module for 
$R$. Then $R$ has rational singularities if and only if for every regular affine $S$ with $R \subseteq S \subseteq K$, one has $\omega_{R} \subseteq \operatorname{Hom}_{R}\left(S, \omega_{R}\right)$ inside $\operatorname{Hom}_{R}\left(K, \omega_{R}\right)$.

Given the lemma, the derivation of the theorem is somewhat technical. Here I simply note that one key idea is to show ([SVdB08, Proposition 2.6]) that if $\Lambda$ is homologically homogeneous of dimension $d$ then $\omega_{\Lambda}=\operatorname{Hom}_{R}(\Lambda, R)$ is an invertible $\Lambda$-module, and furthermore the shift $\omega_{\Lambda}[d]$ is a dualizing complex for $\Lambda$ in the sense of Yekutieli [Yek92]. This result has been extended [Mac10, Theorem 5.1.12] to remove the hypothesis of finite global dimension (so $\Lambda$ is assumed to be "injectively homogeneous") and the hypotheses on the field $k$.

The theorem of Stafford and Van den Bergh does require an assumption on the characteristic of $k$, as they observe [SVdB08, page 671]: there is a homologically homogeneous ring in characteristic 2 with CM center $R$ for which $R$ fails to have rational singularities (in any reasonable sense). The root cause of this bad behavior seems to be the failure of a fixed ring $S^{G}$ to be a direct summand of $S$ in bad characteristic. It is reasonable to ask, then, as Stafford and Van den Bergh do: Suppose $\Lambda$ is a homologically homogeneous ring whose center $R$ is an affine $k$-algebra for a field $k$ of characteristic $p>0$, and assume that $R$ is an $R$-module direct summand of $\Lambda$. Must $R$ have rational singularities?

One application of Theorem 0.1 is to rule out overly optimistic thoughts on the existence of "generalized" non-commutative desingularizations. For example, one might remove the assumption that $\Lambda$ be an $R$-order and simply say that a weak non-commutative desingularization is an $R$-algebra $\Lambda=\operatorname{End}_{R}(M)$, where $M$ is a reflexive $R$-module, such that gldim $\Lambda<\infty$. One might then hope that such things exist quite generally, for, say, every Gorenstein normal domain [MOa]. However, in dimension two this definition would coincide with that of a non-commutative crepant resolution since endomorphism rings of reflexive $R$-modules have depth at least two, so would only exist for rational singularities by Theorem 0.1 . Therefore a counterexample to the hope would be something like $\mathbb{C}[x, y, z] /\left(x^{3}+y^{3}+z^{3}\right)$, which is a Gorenstein normal domain but does not have rational singularities.

\section{P. EXAMPLES: FINITE REPRESENTATION TYPE}

Let $\Lambda$ be an artin algebra of finite representation type, i.e. there are only a finite number of non-isomorphic indecomposable finitely generated $\Lambda$-modules. Auslander defined what is now called the Auslander algebra of $\Lambda$ to be $\Gamma=\operatorname{End}_{\Lambda}\left(M_{1} \oplus \cdots \oplus M_{t}\right)$, where $M_{1}, \ldots, M_{t}$ is a complete set of non-isomorphic indecomposable finitely generated $\Lambda$-modules. By Corollary A.3, $\Gamma$ is Morita equivalent to any other algebra of the form $\operatorname{End}_{\Lambda}(N)$, where $N$ is a representation generator for $\Lambda$, that is, contains every indecomposable finitely generated $\Lambda$-module as a direct summand. These algebras are distinguished by the following result.

Theorem P.1 (Auslander [Aus71]). Let $\Lambda$ be an artin algebra of finite representation type with representation generator $M$. Assume that $\Lambda$ is not semisimple. Set $\Gamma=\operatorname{End}_{\Lambda}(M)$. Then $\operatorname{gldim} \Gamma=2$.

The proof of this theorem is quite direct from Proposition A.4 and the left-exactness of $\operatorname{Hom}_{\Lambda}(M,-)$. Indeed, assume that $\Lambda$ is not semisimple and let $X$ be a finitely generated

$\Gamma$-module, with projective presentation $P_{1} \stackrel{\varphi}{\longrightarrow} P_{0} \longrightarrow X \longrightarrow 0$. The projective modules $P_{i}$ are each of the form $\operatorname{Hom}_{\Lambda}\left(M, M_{i}\right)$ for $\Lambda$-modules $M_{1}$ and $M_{0}$, both in add $M$. Similarly, 
$\varphi=\operatorname{Hom}_{\Lambda}(M, f)$ for some $f: M_{1} \longrightarrow M_{0}$. Put $M_{2}=\operatorname{ker} f$. Then

$$
0 \longrightarrow \operatorname{Hom}_{\Lambda}\left(M, M_{2}\right) \longrightarrow \operatorname{Hom}_{\Lambda}\left(M, M_{1}\right) \stackrel{\operatorname{Hom}_{\Lambda}(M, f)}{\longrightarrow} \operatorname{Hom}_{\Lambda}\left(M, M_{0}\right) \longrightarrow X \longrightarrow 0
$$

is a projective resolution of $X$ of length two.

Auslander and Roggenkamp [AR72] proved a version of this theorem in Krull dimension one, specifically for (classical) orders over complete discrete valuation rings. For their result, define an order $\Lambda$ over a complete DVR $T$ to have finite representation type if there are only a finite number of non-isomorphic indecomposable finitely generated $\Lambda$-modules which are free over $T$; these are called $\Lambda$-lattices. If $M$ contains all indecomposable $\Lambda$-lattices as direct summands, then $\Gamma=\operatorname{End}_{\Lambda}(M)$ is proven to have global dimension at most two; the proof is nearly identical to the one sketched above. One need only observe that the kernel $M_{2}$ of a homomorphism between $\Lambda$-lattices $f: M_{1} \longrightarrow M_{0}$ is again a $\Lambda$-lattice.

In general, say that a (commutative) local ring $R$ has finite representation type, or finite Cohen-Macaulay type, if there are only a finite number of non-isomorphic indecomposable maximal Cohen-Macaulay (MCM) $R$-modules. Recall that when $R$ is complete, a finitely generated $R$-module $M$ is MCM if and only if it is free over a Noether normalization of $R$.

We have already met, in \$ل, the two-dimensional complete local rings of finite representation type, at least over $\mathbb{C}$. By results of Auslander and Esnault [Aus86b, Esn85], they are precisely the quotient singularities $R=\mathbb{C} \llbracket u, v \rrbracket^{G}$, where $G \subset \mathrm{GL}(2, \mathbb{C})$ is a finite group. Moreover, Herzog's Lemma J.5 implies that in that case the power series ring $S=\mathbb{C} \llbracket u, v \rrbracket$ is a representation generator for (the MCM modules over) $R$. Once again the proof above applies nearly verbatim to show (redundantly, cf. Proposition J.1) that $\operatorname{End}_{R}(S)$ has global dimension two.

In dimension three or greater, the kernel $M_{2}=\operatorname{ker}\left(M_{1} \longrightarrow M_{0}\right)$ is no longer a MCM module. When the ring $R$ is $\mathrm{CM}$, however, one can replace it by a high syzygy to obtain the following result.

Theorem P.2 (Iyama [Iya07a], Leuschke [Leu07], Quarles [Qua05]). Let $R$ be a CM local ring of finite representation type and let $M$ be a representation generator for $R$. Set $\Lambda=$ $\operatorname{End}_{R}(M)$. Then $\Lambda$ has global dimension at most $\max \{2, \operatorname{dim} R\}$, and equality holds if $\operatorname{dim} R \geqslant$ 2. More precisely, $\operatorname{pd}_{\Lambda} S=2$ for every simple $\Lambda$-module $S$ except the one corresponding to $R$, which has projective dimension equal to $\operatorname{dim} R$.

Recall that the projective module corresponding to an indecomposable direct summand $N$ of $M$ is $P_{N}=\operatorname{Hom}_{R}(M, N)$, and the corresponding simple module is $S_{N}=P_{N} / \operatorname{rad} P_{N}$.

The proof of the assertion gldim $\Lambda \leqslant \max \{2, \operatorname{dim} R\}$ is exactly similar to the argument sketched above. ${ }^{3}$ For the more precise statement about the projective dimensions of the simple modules, recall that over a CM local ring of finite representation type, every nonfree indecomposable MCM module $X$ has an $A R$ (or almost split) sequence. This is a nonsplit short exact sequence of MCM modules, $0 \longrightarrow Y \longrightarrow E \longrightarrow X \longrightarrow 0$, such that every homomorphism $Z \longrightarrow X$ from a MCM module $Z$ to $X$, which is not a split surjection, factors through $E$. In particular, one can show that if $M$ is a representation generator, then applying

\footnotetext{
${ }^{3}$ In the published version of [Leu07], I gave an incorrect argument for the equality $\operatorname{gldim} \Lambda=\operatorname{dim} R$ if $\operatorname{dim} R \geqslant 2$, pointed out to me by C. Quarles and I. Burban. I claimed that if $S$ is a simple $\Lambda$-module, then a $\Lambda$-projective resolution of $S$ consists of MCM $R$-modules, so has length at least $\operatorname{dim} R$ by the depth lemma. That's not true, since $\Lambda$ isn't MCM. The equality can be rescued by appealing to Proposition F.1 liii).
} 
$\operatorname{Hom}_{R}(M,-)$ to the $\mathrm{AR}$ sequence ending in $X$ yields the exact sequence

$$
0 \longrightarrow \operatorname{Hom}_{R}(M, Y) \longrightarrow \operatorname{Hom}_{R}(M, E) \longrightarrow \operatorname{Hom}_{R}(M, X) \longrightarrow S_{X} \longrightarrow 0,
$$

where $S_{X}$ is the simple $\operatorname{End}_{R}(M)$-module corresponding to $X$. In particular, this displays a projective resolution of $S_{X}$ for every non-free indecomposable MCM module $X$. The simple $S_{R}$ corresponding to $R$ is thus very special, and has projective dimension equal to $\operatorname{dim} R$ by Proposition F.1.(iii). Observe that this argument relies essentially on the fact that $R$ has a representation generator; below is an example where $\operatorname{pd}_{\Lambda} S>\operatorname{dim} R$ for a simple $S$ even though $\Lambda$ has finite global dimension.

Among other things, the statement about simple modules implies that when $\operatorname{dim} R \geqslant 3$, the endomorphism ring of a representation generator is never homologically homogeneous, so is never a non-commutative crepant resolution. A concrete example of this failure has already appeared in Example M.5. Here is another example in the non-Gorenstein case.

Example P.3 ([Leu07, Example 12], [SQ05]). Let $k$ be an infinite field and let $R$ be the complete scroll of type $(2,1)$, that is, $R=k \llbracket x, y, z, u, v \rrbracket / I$, where $I$ is generated by the $2 \times 2$ minors of the matrix $\left(\begin{array}{lll}x & y & u \\ y & z & v\end{array}\right)$. Then $R$ is a three-dimensional CM normal domain which is not Gorenstein, and has finite representation type [AR89]. The only non-free indecomposable MCM modules are, up to isomorphism,

- the canonical module $\omega \cong(u, v) R$;

- the first syzygy of $\omega$, isomorphic to $\omega^{*}=\operatorname{Hom}_{R}(\omega, R)$ and to $(x, y, u) R$;

- the second syzygy $N$ of $\omega$, rank two and 6-generated; and

- the dual $L=\operatorname{Hom}_{R}\left(\omega^{*}, \omega\right)$ of $\omega^{*}$, isomorphic to $(x, y, z) R$.

By Theorem P.2, $\Lambda=\operatorname{End}_{R}\left(R \oplus \omega \oplus \omega^{*} \oplus N \oplus L\right)$ has global dimension 3. However, $\Lambda$ is not MCM as an $R$-module, since none of $L^{*}, N^{*}$, and $\operatorname{Hom}_{R}\left(\omega, \omega^{*}\right)$ is MCM. One can check with, say, Macaulay2 [GS] that $\operatorname{End}_{R}(R \oplus \omega)$ and $\operatorname{End}_{R}\left(R \oplus \omega^{*}\right)$ are up to Morita equivalence the only endomorphism rings of the form $\operatorname{End}_{R}(D)$, with $D$ non-free MCM, that are themselves MCM. In fact $\operatorname{End}_{R}(R \oplus \omega) \cong \operatorname{End}_{R}\left(R \oplus \omega^{*}\right)$ as rings.

Set $\Gamma=\operatorname{End}_{R}(R \oplus \omega)$. Then $\Gamma$ has two simple modules $S_{\omega}$ and $S_{R}$. Using Lemma M.2 and the known structure of the AR sequences over $R$, Smith and Quarles [SQ05] show that $\operatorname{pd}_{\Gamma} S_{\omega}=4$ and $\operatorname{pd}_{\Gamma} S_{R}=3$. Thus $\Gamma$ has global dimension equal to 4 by Proposition F.1 (iii), but is not a non-commutative crepant resolution of $R$.

Example P.4. There is only one other known example of a non-Gorenstein CM complete local ring of finite representation type in dimension three or more. It is the (completion of the) homogeneous coordinate ring of the cone over the Veronese embedding $\mathbb{P}^{2} \hookrightarrow \mathbb{P}^{5}$. Explicitly, set $R=\mathbb{C} \llbracket x^{2}, x y, x z, y^{2}, y z, z^{2} \rrbracket \subset \mathbb{C} \llbracket x, y, z \rrbracket=S$. Then the indecomposable non-free MCM $R$-modules are the canonical module $\omega=\left(x^{2}, x y, x z\right) R$ and its first syzygy $N$. Observe that $S \cong R \oplus \omega$ as $R$-modules, so by Theorem J.2. $\operatorname{End}_{R}(R \oplus \omega) \cong S \#\left(\mathbb{Z}_{2}\right)$ has finite global dimension. Since $\operatorname{End}_{R}(S) \cong S \oplus S, \Lambda=\operatorname{End}_{R}(S)$ is a non-commutative crepant resolution for $R$.

By Theorem P.2, $\Gamma=\operatorname{End}_{R}(R \oplus \omega \oplus N)$ has global dimension 3. But $\operatorname{Hom}_{R}(N, R)$ and $\operatorname{Hom}_{R}(N, N)$ have depth 2 , so $\Gamma$ is not a non-commutative crepant resolution.

\section{Q. EXAmple: THE GENERIC DETERMinANT}

The most common technique thus far for constructing non-commutative crepant resolutions has been to exploit a known (generally crepant) resolution of singularities and a tilting 
object on it. In fact, the basic technique is already present in Van den Bergh's proof of Theorem K.11. This has been used in several other families of examples. This $\S$ is devoted to describing a particular example of this technique in action, namely the generic determinantal hypersurface ring.

Let $k$ be a field and $X=\left(x_{i j}\right)$ the generic square matrix of size $n \geqslant 2$, whose entries $x_{i j}$ are thus a family of $n^{2}$ indeterminates over $k$. Set $S=k[X]=k\left[\left\{x_{i j}\right\}\right]$ and let $R$ be the hypersurface ring $S /(\operatorname{det} X)$ defined by the determinant of $X$. Then $R$ is a normal Gorenstein domain of dimension $n^{2}-1$.

Fix a free $S$-module $\mathscr{F}$ of rank $n$. Left-multiplication with the matrix $X$ naturally defines the generic $S$-linear map $\mathscr{F} \longrightarrow \mathscr{F}$. The exterior powers $\wedge^{a} X: \wedge^{a} \mathscr{F} \longrightarrow \wedge^{a} \mathscr{F}$ define natural $S$-modules

$$
M_{a}=\operatorname{cok} \wedge^{a} X
$$

for $a=1, \ldots, n$. In fact each $M_{a}$ is annihilated by $\operatorname{det} X$, so is naturally an $R$-module. The pair $\left(\bigwedge^{a} X, \bigwedge^{n-a} X^{T}\right)$ forming a matrix factorization of $\operatorname{det} X$, the $M_{a}$ are even MCM modules over $R$ [Eis80]. They are in particular reflexive, of rank $\left(\begin{array}{c}n-1 \\ a-1\end{array}\right)$.

Set $M=\bigoplus_{a=1}^{n} M_{a}$ and $\Lambda=\operatorname{End}_{R}(M)$. The crucial result of [BLV10b], in this case, is then

Theorem Q.1. The $R$-algebra $\Lambda$ provides a non-commutative crepant resolution of $R$.

The proof in [BLV10b] proceeds by identifying the $M_{a}$ as geometric objects with tilting in their ancestries, as follows. Let $F$ be a $k$-vector space of dimension $n$, and set $\mathbb{P}=\mathbb{P}\left(F^{\vee}\right) \cong \mathbb{P}_{k}^{n-1}$ be the projective space over $R$, viewed as equivalence classes [ $\left.\lambda\right]$ of linear forms $\lambda: F \longrightarrow k$. Put

$$
Y=\mathbb{P} \times \operatorname{Spec} S,
$$

with canonical projections $\tilde{p}: Y \longrightarrow \mathbb{P}$ and $\widetilde{q}: Y \longrightarrow \operatorname{Spec} S$. Identify Spec $S$ with the space of $(n \times n)$ matrices $A$ over the field $k$, with coordinate functions given by the indeterminates $x_{i j}$. Then the incidence variety

$$
Z=\{([\lambda], A) \mid \text { image } A \subseteq \operatorname{ker} \lambda\}
$$

is a resolution of singularities of $\operatorname{Spec} R$. (Compare with Example H.1, which is the case $n=2$.) Indeed, the image of $Z$ under $\widetilde{q}: Y \longrightarrow \operatorname{Spec} S$ is precisely the locus of matrices $A$ with $\operatorname{rank} A<n$, that is, Spec $R$. Furthermore, the singular locus of $\operatorname{Spec} R$ consists of the matrices of $\operatorname{rank}<n-1$, and $q:=\left.\widetilde{q}\right|_{Z}: Z \longrightarrow \operatorname{Spec} R$ is an isomorphism away from these points. One can explicitly write down the equations cutting $Z$ out of $Y$, and verify that $Z$ is smooth, and is a complete intersection in $Y$; if in particular $j: Z \longrightarrow Y$ is the inclusion, then this implies that $j_{*} \mathscr{O}_{Z}$ is resolved over $\mathscr{O}_{Y}$ by a Koszul complex on the Euler form $F \otimes_{k} \mathscr{O}_{Y}(-1) \longrightarrow \mathscr{O}_{Y}$.

Here is a pictorial description of the situation.

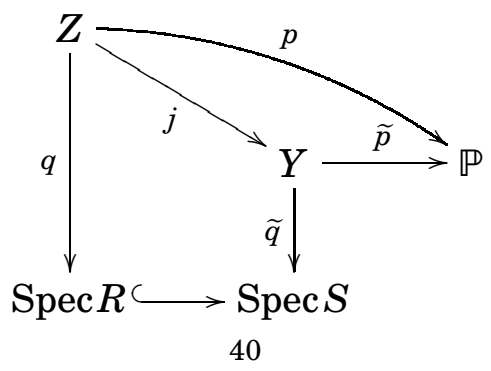


Recall from $\S \mathrm{E}$ that $T=\bigoplus_{a=1}^{n} \Omega^{a-1}(a)$, where $\Omega=\Omega_{\mathbb{P} / k}$ is the sheaf of differential forms on $\mathbb{P}$ and $\Omega^{j}=\wedge^{j} \Omega$, is a tilting bundle on $\mathbb{P}$. Set $\mathscr{M}_{a}=p^{*} \Omega^{a-1}(a)$ for $a=1, \ldots, n$, a locally free sheaf on the resolution $Z$. As the typography hints, $\mathcal{M}_{a}$ is a geometric version of $M_{a}$, in the following sense.

Proposition Q.2. As $R$-modules, $\mathbf{R} q_{*} \mathscr{M}_{a}=M_{a}$. More precisely, $\mathbf{R}^{j} q_{*} \mathscr{M}_{a}=0$ for $j>0$ and $q_{*} \mathscr{M}_{a}=M_{a}$ for all $a$.

The proof of the Proposition involves juggling two Koszul complexes. Tensoring (E.1) with $\mathscr{O}_{\mathbb{P}}(a)$ and truncating gives an exact sequence

$$
0 \longrightarrow \Omega^{a-1}(a) \longrightarrow \bigwedge^{a-1} F \otimes_{k} \mathscr{O}_{\mathbb{P}}(1) \longrightarrow \cdots \longrightarrow F \otimes_{k} \mathscr{O}_{\mathbb{P}}(a-1) \longrightarrow \mathscr{O}_{\mathbb{P}}(a) \longrightarrow 0
$$

The projection $p$ being flat, the pullback $p^{*}$ is exact, yielding

$$
0 \longrightarrow \mathscr{M}_{a} \longrightarrow \bigwedge^{a-1} F \otimes_{\mathscr{O}_{Z}}(1) \longrightarrow \cdots \longrightarrow F \otimes_{k} \mathscr{O}_{Z}(a-1) \longrightarrow \mathscr{O}_{Z}(a) \longrightarrow 0
$$

Compute $\mathbf{R} q_{*}$ as $\mathbf{R} \widetilde{q}_{*} j_{*}$. As $j_{*} \mathscr{O}_{Z}$ is resolved over $\mathscr{O}_{Y}$ by a Koszul complex, we may replace the former with the latter and obtain a double complex in the fourth quadrant, with $\wedge^{a-1} F \otimes_{k} \mathscr{O}_{Y}(1)$ at the origin and $\wedge^{a-i+1} F \otimes_{k} \wedge^{-j} F \otimes_{k} \mathscr{O}_{Y}(i+j+1)$ in the $(i, j)$ position. Now apply $\mathbf{R} q_{*}$. By [Har77, Ex. III.8.4], the higher direct images of the projective bundle $q: Y \longrightarrow \operatorname{Spec} S$ are completely known,

$$
\mathbf{R}^{j} \widetilde{q}_{*} \mathscr{O}_{Y}(t)= \begin{cases}0 & \text { if } t<0 \text { or } 1<j<n-1 \\ \operatorname{Sym}_{t}(F) \otimes_{k} S=\operatorname{Sym}_{t}(\mathscr{F}) & \text { for } j=0 ; \text { and } \\ 0 & \text { for } j=n-1 \text { if } t \geqslant-n\end{cases}
$$

This already proves $\mathbf{R}^{j} q_{*} \mathscr{M}_{a}=0$ for $j>0$, and allows one to represent $q_{*} \mathscr{M}_{a}$ by the homology of the total complex of the following double complex of free $S$-modules. (For notational 
simplicity write $\bigwedge^{i}$ and $\operatorname{Sym}_{j}$ instead of $\bigwedge^{i} \mathscr{F}$ and $\left.\operatorname{Sym}_{j} \mathscr{F}.\right)$

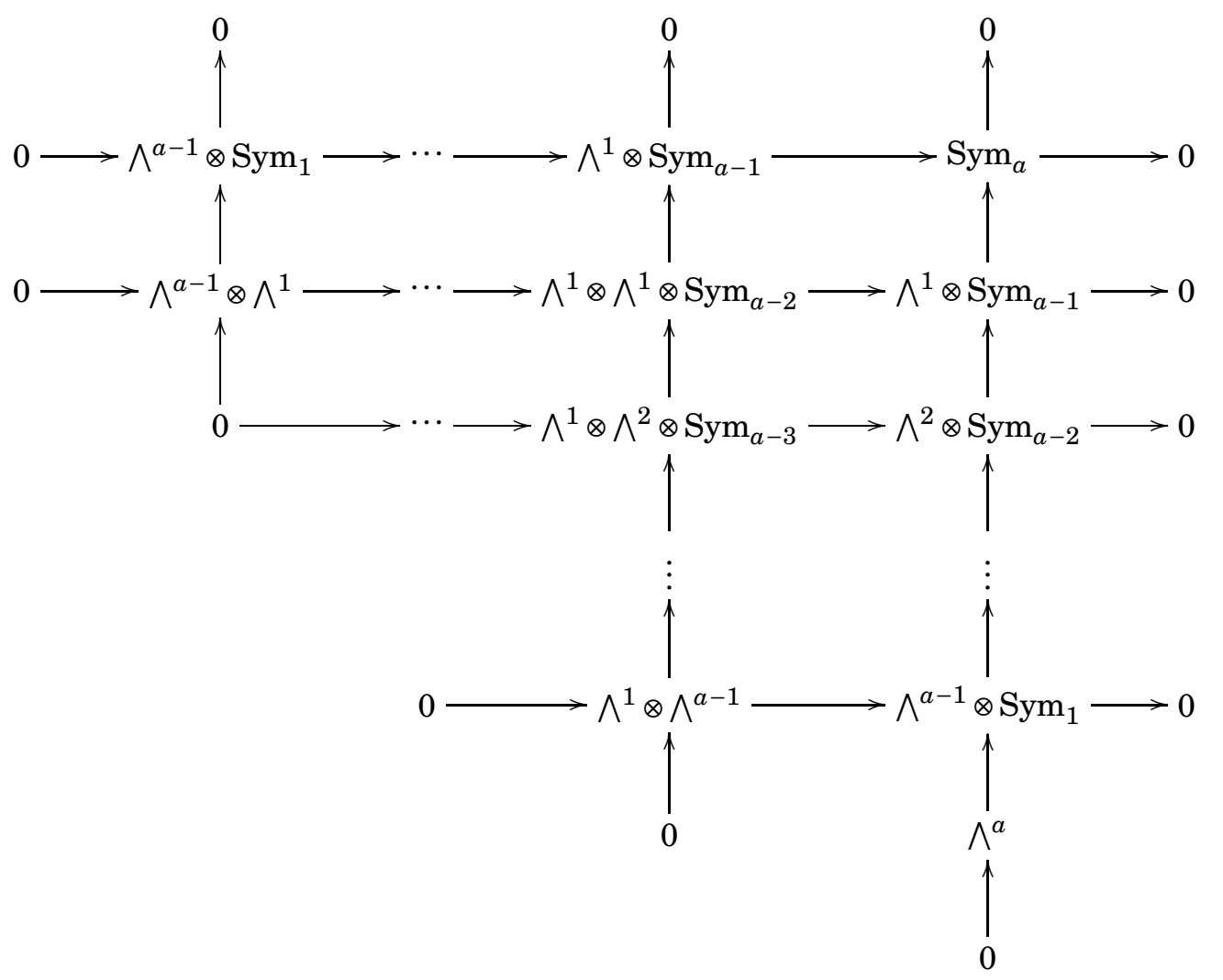

Here the $j^{\text {th }}$ column is obtained by tensoring the strand of degree $j$ in the Koszul complex with $\wedge^{a-j-1} \mathscr{F}$, so is acyclic [Eis95, A2.10]. Similarly, the $(-i)^{\text {th }}$ row is the degree $a$ strand in a Koszul complex tensored with $\bigwedge^{i} \mathscr{F}$, and so is exact with the exceptions of the top and bottom rows. The top row has homology equal to $\wedge^{a} \mathscr{F}$ at the leftmost end, while the bottom row has homology $\wedge^{a} \mathscr{F}$ on the right. One checks from the explicit nature of the maps that the total complex is thus reducible to $\bigwedge^{a} X: \wedge^{a} \mathscr{F} \longrightarrow \bigwedge^{a} \mathscr{F}$, whence $q_{*} \mathscr{M}_{a}=M_{a}$, as claimed.

Now it is relatively easy to prove that

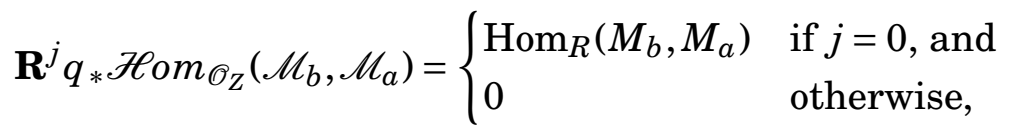

and to establish the rest of the assertions in the next theorem.

Theorem Q.3. The object $\mathbf{R} q_{*} \mathscr{H}^{\circ} \mathrm{m}_{\mathscr{O}_{Z}}\left(\mathscr{M}_{b}, \mathscr{M}_{a}\right)$ is isomorphic in the bounded derived category $\mathrm{D}^{b}(S$-mod) to a single morphism between free $S$-modules situated in (cohomological) degrees -1 and 0 . Therefore the $R$-module $q_{*} \mathscr{H}$ om $\mathscr{O}_{Z}\left(\mathscr{M}_{b}, \mathscr{M}_{a}\right)=\operatorname{Hom}_{R}\left(M_{b}, M_{a}\right)$ is a MCM $R$-module and the higher direct images vanish, so that in particular

$$
\mathbf{R}^{1} q_{*} \mathscr{H}_{0} m_{\mathscr{O}_{Z}}\left(\mathscr{M}_{b}, \mathscr{M}_{a}\right)=\operatorname{Ext}_{R}^{1}\left(M_{b}, M_{a}\right)=0 .
$$

It remains to see that $\oplus_{a=1}^{n} \mathscr{M}_{a}$ is a tilting object on $Z$, so that $\Lambda=\operatorname{End}_{R}\left(\oplus_{a} M_{a}\right)=$ $q_{*} \mathscr{E} n d_{\mathscr{O}_{Z}}\left(\oplus_{a} \mathscr{M}_{a}\right)$ has finite global dimension, whence is a non-commutative crepant resolution of $R$. It suffices for this to compute the cohomology

$$
H^{i}\left(\mathbb{P}, \mathscr{C o m}_{\mathscr{O}_{\mathbb{P}}}\left(\Omega_{42}^{b-1}(b), \Omega^{a-1}(a)\right)(c)\right) ;
$$


since $p$ is flat, this will compute $\operatorname{Ext}_{\mathscr{O}_{Z}}^{i}\left(\mathscr{M}_{b}, \mathscr{M}_{a}\right)$ as well. In [BLV10b] we gave a characteristicfree proof of this vanishing, and another appears in the appendix by Weyman to [ESW03]. In characteristic 0, one can compute the cohomology with Bott vanishing [Wey03, Chapter 4]. This allows the following proposition and theorem.

Proposition Q.4. The $\mathscr{O}_{Z}$-module $\mathscr{M}=\bigoplus_{a} \mathscr{M}_{a}=\bigoplus_{a=1}^{n} p^{*} \Omega^{a-1}(a)$ is a tilting bundle in $\mathrm{D}^{b}(\operatorname{coh} Z)$. In detail, with $\mathscr{A}=\operatorname{End}_{\mathrm{D}^{b}(\operatorname{coh} Z)}(\mathscr{M})$,

(i) $\operatorname{Ext}_{\mathscr{O}_{Z}}^{i}(\mathscr{M}, \mathscr{M}):=\operatorname{Hom}_{\mathrm{D}^{b}(\operatorname{coh} Z)}(\mathscr{M}, \mathscr{M}[i])=0$ for $i>0$;

(ii) $\mathbf{R H o m}_{\mathscr{O}_{Z}}(\mathscr{M},-): \mathrm{D}^{b}(\operatorname{coh} Z) \longrightarrow \mathrm{D}^{b}(\mathscr{A}$-mod $)$ is an equivalence of triangulated categories, with $-\stackrel{\mathbf{L}}{\otimes} \mathscr{A} \mathscr{M}$ as inverse;

(iii) $\mathscr{A}$ has finite global dimension.

(iv) $\mathscr{A} \cong \Lambda=\operatorname{End}_{R}(M)$.

Theorem Q.5. Let $k$ be a field, $X$ an $(n \times n)$ matrix of indeterminates, $n \geqslant 2$, and $R=$ $k[X] /(\operatorname{det} X)$ the generic determinantal hypersurface ring. Let $M_{a}=\operatorname{cok} \wedge^{a} X$ for $a=1, \ldots, n$, and put $M=\bigoplus_{a} M_{a}$. Then the $R$-algebra $\Lambda=\operatorname{End}_{R}(M)$ has finite global dimension and is $M C M$ as an $R$-module. It is in particular a non-commutative crepant resolution of $R$.

In [BLV10b] we replace the square matrix $X$ by an $(m \times n)$ matrix with $n \geqslant m$ and $R$ with the quotient by the maximal minors $k[X] / I_{m}(X)$, which defines the locus in Spec $k[X]$ of matrices with non-maximal rank. The same construction $M_{a}=\operatorname{cok} \wedge^{a} X$ yields an algebra $\Lambda=\operatorname{End}_{R}\left(\bigoplus_{a=1}^{m} M_{a}\right)$ which is still MCM as an $R$-module and still has finite global dimension. In this case, however, $\Lambda$ is not a non-singular $R$-algebra, so not a non-commutative crepant resolution according to our definition. This is directly attributable to the fact that quotients by minors are Gorenstein if and only if $n=m$, so that Corollary $\bar{K}$.3 fails for non-square matrices. In a forthcoming paper [BLV11], we establish the same result for the quotient by arbitrary minors $k[X] / I_{t}(X)$, with $1 \leqslant t \leqslant m$, using a tilting bundle on the Grassmannian [BLV10a]. In particular we obtain non-commutative crepant resolutions when the matrix is square.

Similar techniques, i.e. constructions using tilting objects on known resolutions of singularities, are used by Kuznetsov [Kuz08] to give non-commutative desingularizations for several more classes of examples, including cones over Veronese/Segre embeddings and Grassmannians, as well as Pfaffian varieties.

\section{R. NON-COMmUtATIVE BLOWUPS}

It was clear from early on in the development of non-commutative (projective) geometry that it would be most desirable to have a non-commutative analogue of the most basic birational transformation, the blowup. This $\S$ sketches a few approaches to the problem.

First recall that if $R$ is a commutative ring and $I$ is an ideal of $R$, the blowup $\mathscr{B}_{I}(X)$ of $X=\operatorname{Spec} R$ at (the closed subscheme defined by) $I$ is Proj $R[I t]$, where $R[I t]$ is the Rees algebra $R \oplus I \oplus I^{2} \oplus \cdots$. The exceptional locus of the blowup is the fiber cone Proj $R[I t] / I R[I t]=$ $R / I \oplus I / I^{2} \oplus \cdots$.

One might hope to mimic this definition for sufficiently nice non-commutative rings. This turns out to give unsatisfactory results. For example ([Art97], [VdB01, page 2]) set $\Lambda=$ $k\langle x, y\rangle /(y x-x y-y)$, and consider the ideal $\mathfrak{m}=(x, y)$ "corresponding" to the origin of this 
non-commutative surface. Then $\mathfrak{m}^{n}=\left(x^{n}, y\right)$ for all $y$, so the fiber cone $R[\mathfrak{m} t] / \mathfrak{m} R[\mathfrak{m} t]$ is onedimensional in each degree, and is isomorphic to $k[z]$. This means that the exceptional locus is in some sense zero-dimensional, whereas one should expect the exceptional divisor of a blowup of a point in a surface to have dimension 1.

Van den Bergh [VdB01] constructs an analogue of the Rees algebra directly over projective quasi-schemes $\operatorname{Proj} \Lambda$ (see $\S F$ ). Specifically, if $X=\operatorname{Proj} \Lambda$ is a quasi-scheme, he gives a construction of the blowup of a smooth point $p$ in a commutative curve $Y$ contained in $X$. (This means that $\mathrm{Q} c h Y \simeq \operatorname{Proj}(\Lambda / x \Lambda)$ for some $x \in \Lambda$.) Using this construction, Van den Bergh considers blowups of quantum projective planes at small numbers of points, in particular non-commutative deformations of the del Pezzo surfaces obtained by blowing up in $\leqslant 8$ points. I won't go into the details of the construction or the applications here.

There is a more recent proposal for a definition of the phrase "non-commutative blowup," which is inspired by the classic flop of Example I.2 and by Theorem L.1. In general, the idea is that for an ideal $I$ of a ring $\Lambda$, the non-commutative blowup of $\Lambda$ in $I$ is the ring

$$
\mathscr{B}_{I}^{\mathrm{nc}}(\Lambda)=\operatorname{End}_{\Lambda}(\Lambda \oplus I) .
$$

In the situation of Example $\left[.2\right.$, we saw that $\mathscr{B}_{I}^{\mathrm{nc}}(R)$ was derived equivalent to the usual blowup $\mathscr{B}_{I}(\operatorname{Spec} R)$. Thus suggests the following question, a version of which I first heard from R.-O. Buchweitz.

Question R.1. Can one generalize or imitate the normalization algorithm of $\$$ Lto show that there is a sequence of non-commutative blowups starting with $\Lambda=\Lambda_{0}$ and continuing with $\Lambda_{i+1}=\mathscr{B}_{I_{i}}^{\mathrm{nc}}\left(\Lambda_{i}\right)=\operatorname{End}_{\Lambda_{i}}\left(\Lambda_{i} \oplus I_{i}\right)$ for some ideals $I_{i} \subset \Lambda_{i}$, such that $\Lambda_{i}$ eventually has finite global dimension?

One might try to follow Hironaka and blow up only in "smooth centers," i.e. assume that $\Lambda_{i} / I_{i}$ is non-singular, as is the case in Example I.2.

Very recent work of Burban-Drozd [BD11] confirms that the non-commutative blowup as above is a sort of categorical desingularization whenever $X$ is a reduced algebraic curve singularity having only nodes and cusps for singular points, and $I$ is the conductor ideal. They observe that $\mathscr{A}=\mathscr{E} n d_{\mathscr{O}_{X}}\left(\mathscr{O}_{X} \oplus I\right)$ has global dimension equal to 2 , that $\left(\mathscr{O}_{X} \oplus I\right) \otimes_{\mathscr{O}_{X}}$ -: $\operatorname{coh} X \longrightarrow \mathscr{A}$-mod is fully faithful, and that $\operatorname{Hom}_{\mathscr{A}}\left(\mathscr{O}_{X} \oplus I,-\right): \mathscr{A}$-mod $\longrightarrow \operatorname{coh} X$ is exact.

In his Master's thesis, Quarles constructs a direct connection between blowups and noncommutative blowups, the only one I know of. Let $(R, \mathfrak{m}, k)$ be a Henselian local $k$-algebra, with $k$ an algebraically closed field. Let $I$ be an ideal of $R$ which is MCM and reflexive as an $R$-module, and set $\Lambda=\mathscr{B}_{I^{*}}^{\text {nc }}(R)=\operatorname{End}_{R}\left(R \oplus I^{*}\right)$. Then Quarles defines [Qua05, Section 7] a bijection between the closed points of $\mathscr{B}_{I}(\operatorname{Spec} R)=\operatorname{Proj} R[I t]$ and the set of indecomposable $\Lambda$-modules $X$ arising as extensions $0 \longrightarrow S_{R} \longrightarrow X \longrightarrow S_{I^{*}} \longrightarrow 0$ of the two simple modules $S_{R}$ and $S_{I}$. The bijection is just as sets, and carries no known algebraic information; in particular, it is not known to be a moduli space.

There are some immediate problems. For example, in Example P.3 we have $\operatorname{End}_{R}(R \oplus \omega) \cong$ $\operatorname{End}_{R}\left(R \oplus \omega^{*}\right)$, but $R[\omega t] \not \nexists R\left[\omega^{*} t\right]$, since one is regular and the other is not. The associated projective schemes are isomorphic, of course. It is not clear how to reconcile this.

A similar approach has been suggested in prime characteristic [Tod09, Tod08b, Tod08a, TY09, Yas09]. For the rest of this $\S$ let $k$ be an algebraically closed field of characteristic $p>$ 0 . Let $X$ and $Y$ be normal algebraic schemes over $k$, and let $f: Y \longrightarrow X$ be a finite dominant 
morphism. Then Yasuda [Yas09] proposes to call the endomorphism ring $\mathscr{E} n d_{\mathscr{O}_{X}}\left(f_{*} \mathscr{O}_{Y}\right)$ the non-commutative blowup attached to $f$.

In particular, consider the non-commutative blowup of the Frobenius. For every $e \geqslant 1$, set $X_{e}=X$ and let $F_{X}^{e}: X_{e} \longrightarrow X$ be the $e^{\text {th }}$ iterate of the Frobenius morphism. Assume that $F_{X}$ is finite. Then the non-commutative blowup of the $e^{\text {th }}$ Frobenius, $\mathscr{E}_{n} d_{\mathscr{O}_{X}}\left(F_{X *}^{e} \mathscr{O}_{X_{e}}\right)$ is locally given by $\operatorname{End}_{R}\left(R^{1 / p^{e}}\right)$, where $R^{1 / p^{e}}$ is the ring of $\left(p^{e}\right)^{\text {th }}$ roots of elements of $R$. It is isomorphic to $\operatorname{End}_{R^{p^{e}}}(R)$, where now $R^{p^{e}}$ is the subring of $\left(p^{e}\right)^{\text {th }}$ powers. The ring $\operatorname{End}_{R^{p^{e}}}(R)$ consists of differential operators on $R$ [SVdB97] and is sometimes a non-commutative crepant resolution of $R$.

Theorem R.2 (Toda-Yasuda [TY09]). Let $R$ be a complete local ring of characteristic $p$ which is one of the following.

(i) a one-dimensional domain;

(ii) the $A D E$ hypersurface singularity of type $\left(A_{1}\right)$ (and $p \neq 2$ ); or

(iii) a ring of invariants $k \llbracket x_{1}, \ldots, x_{n} \rrbracket^{G}$, where $G \subset \mathrm{GL}(n, k)$ is a finite subgroup with order invertible in $k$.

Then for $e \gg 0, \operatorname{End}_{R}\left(R^{1 / p^{e}}\right)$ has finite global dimension. However it is not generally MCM as an $R$-module, so is not a non-commutative crepant resolution.

Let me make a few comments on the proofs. For (i), consider the integral closure $S \cong k \llbracket x \rrbracket$ of $R$. Then for any $e \geqslant 1$, one checks that $\operatorname{End}_{R^{p^{e}}}\left(S^{p^{e}}\right)=\operatorname{End}_{S^{p^{e}}}\left(S^{p^{e}}\right)=S^{p^{e}}$. Take $e$ large enough that $S^{p^{e}} \subseteq R$. Then $R$ is free over $S^{p^{e}}$ of $\operatorname{rank} p^{e}$, so $\operatorname{End}_{R^{p^{e}}}(R) \cong M_{p^{e}}\left(\operatorname{End}_{R^{p^{e}}}\left(S^{p^{e}}\right)\right)=$ $M_{p^{e}}\left(S^{p^{e}}\right)$. This is Morita equivalent to $S^{p^{e}} \cong S$, so has global dimension equal to 1 . It is also clearly MCM.

For (ii), assume $p \neq 2$ and set $R=k \llbracket x_{1}, \ldots, x_{d} \rrbracket /\left(x_{1}^{2}+\cdots+x_{d}^{2}\right)$. Then one can show that for all $e \geqslant 1, R$ is a representation generator for $R^{p^{e}}$. (This requires separate arguments for $d$ odd/even.) By Theorem P.2, $\operatorname{End}_{R^{p^{e}}}(R)$ has finite global dimension. It is not a noncommutative crepant resolution by Theorem M.4.

Finally, for (iii), Toda and Yasuda use results of Smith and Van den Bergh to show that if $S=k \llbracket x_{1}, \ldots, x_{d} \rrbracket$ and $R=S^{G}$ as in the statement, then for $e \gg 0$ every module of covariants $\left(S \otimes_{k} W\right)^{G}$ appears as an $R^{p^{e}}$-direct summand in $R$, in $S$, and in $S^{p^{e}}$. Thus $\operatorname{End}_{R^{p^{e}}}\left(S^{p^{e}}\right) \cong S^{p^{e}} \# G\left(\right.$ Theorem J.2) is Morita equivalent to $\operatorname{End}_{R^{p^{e}}}(R)$ by Corollary A.3, and they simultaneously have finite global dimension.

In general, there are non-trivial obstructions to $\operatorname{End}_{R}\left(R^{1 / p^{e}}\right)$ being a non-commutative crepant resolution. For example, Dao points out [Dao10] that when $R$ is a complete intersection ring, $R^{1 / p^{e}}$ is known to be Tor-rigid [AM01], so if $R$ satisfies $\left(R_{2}\right)$ then $\operatorname{End}_{R}\left(R^{1 / p^{e}}\right)$ is not MCM for any $e \geqslant 1$ by the discussion following Proposition M.3.

\section{S. OMISSIONS AND OPEN QUESTIONS}

In addition to the examples already mentioned in previous $\S \S$, there is a large and growing array of examples of non-commutative crepant resolutions and related constructions. Lack of space and expertise prevent me from describing them in full, but here are a few references and comments.

Deformations of the Kleinian singularities $\mathbb{C}^{2} / G$, with $G \subset \operatorname{SL}(2, \mathbb{C})$, have non-commutative crepant resolutions [GS04, which are identified as deformed preprojective algebras in the sense of [CBH98]. 
In a different direction, Wemyss has considered the non-Gorenstein case of the classical McKay correspondence, where $G \not \subset \mathrm{SL}(2, \mathbb{C})$ [Wem07, Wem09a, Wem09b]; much of Theorem J.6 breaks down, but much can be recovered by restricting to the so-called "special" representations. This leads to the reconstruction algebra, which is the endomorphism ring of the special MCM modules.

Beil [Bei08] shows that square superpotential algebras, which are certain quiver algebras with relations coming from cyclic derivatives of a superpotential, are non-commutative crepant resolutions of their centers (which are three-dimensional toric Gorenstein normal domains). In fact, Broomhead [Bro09] constructs a non-commutative crepant resolution for every Gorenstein affine toric threefold, from superpotential algebras called dimer models. Similar algebras associated to brane tilings have non-commutative crepant resolutions as well [Moz09, BM09].

Finally, Bezrukavnikov [Bez06] constructs a non-commutative version of the Springer resolution $Z$ from (Q.1), which is different from that in [BLV10b].

Many other topics have been omitted that could have played a role. For example, I have said nothing about (semi-)orthogonal decompositions of triangulated categories and exceptional sequences. These grew out of Beylinson's result in $\$ \mathrm{E}$, via the Rudakov seminar [Rud90]. See [Huy06, Chapter 1] or [B095].

Connections of this material with string theory appear at every turn [SW99]. For example, the derived category $\mathrm{D}(X)$ appears in string theory as the category of branes propagating on the space $X$. Non-commutativity arises naturally in this context from the fact that open strings can be glued together in two different ways, unlike closed strings [BL01]. Furthermore, the Calabi-Yau condition of $\S \mathbb{\mathrm { K }}$ is essential to the string-theoretic description of spacetime [Bro09, OY09]. Most obviously, high energy physics has been a driving force in non-commutative desingularizations and the higher geometric McKay correspondence. I am not competent to do more than gesture at these connections.

I end the article with a partial list of open problems. Some of these are mentioned in the text, while others are implicit.

(1) Conjecture $\mathrm{H} .3$ of Bondal and Orlov, that a generalized flop between smooth varieties induces a derived equivalence, is still largely open outside of dimension three. The related Conjecture K.12 of Van den Bergh, which asks for derived equivalence of both geometric and non-commutative crepant resolutions, is similarly open. See [IW11] for some very recent progress on the non-commutative side.

(2) Existence of a non-commutative crepant resolution is not equivalent to existence of a crepant resolution of singularities in dimension four or higher. See the end of Example G.6 for examples with non-commutative resolutions but no geometric ones, and Example M.5 for failure of the other direction. However, it still may hold in general in dimension three. One might also be optimistic and ask for additional hypotheses to rescue the case of dimension four.

(3) Various results in the text fail for rings that are not Gorenstein, notably Corollary K.3 and Proposition K.7. Is there a better definition of non-commutative crepant resolutions 
which would satisfy these statements over non-Gorenstein Cohen-Macaulay rings? Perhaps we should not expect one, since crepant resolutions of singularities exist only in the Gorenstein case.

On a related note, is symmetry (Definition K.2) too strong a condition? The relevant property in [SVdB08] is that $\operatorname{Hom}_{R}(\Lambda, R)$ is an invertible $(\Lambda-\Lambda)$-bimodule, rather than insisting that $\operatorname{Hom}_{R}(\Lambda, R) \cong \Lambda$. This would, unfortunately, rule out endomorphism rings $\operatorname{End}_{R}(M)$, since they are automatically symmetric by Theorem K.6.ii). Or perhaps the appropriate generalization to non-Gorenstein rings is that $\operatorname{Hom}_{R}\left(\Lambda, \omega_{R}\right)=\Lambda$.

(4) Crepant resolutions of singularities are very special: they exist only for canonical singularities, not in general for terminal singularities. The non-commutative version is more general. One might therefore hope that Theorem K.11 is true for canonical threefolds as well. Van den Bergh's proof of Theorem K.11 applies verbatim for any canonical threefold admitting a crepant resolution of singularities with one-dimensional fibers.

(5) In nearly all of the examples of non-commutative crepant resolutions, the module $M$ such that $\Lambda=\operatorname{End}_{R}(M)$ can be taken maximal Cohen-Macaulay. Lemma M.2 indicates one obstruction to $M$ having high depth. Are there general situations where a noncommutative crepant resolution exists, but no MCM module will suffice? Or situations (other than surfaces) where every non-commutative crepant resolution is given by a MCM module? See [IW10, 5.12] for one result in this direction.

(6) Van den Bergh points out in [VdB04a] that one might try to build a theory of rational singularities for non-commutative rings, extending the results of $\$ \mathrm{O}$. It would be essential to have a non-commutative analogue of the Grauert-Riemenschneider Vanishing theorem (G.2), but none seems to be known. There is an algebraic reformulation of GR Vanishing due to Sancho de Salas [SdS87], cf. [Hun96, Chapter 5]: Let $R$ be a reduced CM local ring essentially of finite type over an algebraically closed field of characteristic zero, and let $I$ be an ideal of $R$ such that Proj $R[I t]$ is smooth; then the associated graded ring $\operatorname{gr}_{I^{n}}(R)$ is Cohen-Macaulay for $n \gg 0$. It would be very interesting to have a purely algebraic proof of this result, particularly if it encompassed some non-commutative rings. The proof of Sancho de Salas uses results from [GR70], so relies on complex analysis; see [HM99] for some progress toward an algebraic proof in dimension two.

(7) In a similar direction, Question R.1 asks for an algorithm to resolve singularities via a sequence of "non-commutative blowups." For a start, one needs any non-trivial connection between $\mathrm{D}^{b}(\operatorname{coh} \operatorname{Proj} R[I t])$ and $\mathrm{D}^{b}\left(\operatorname{End}_{R}(R \oplus I)\right.$-mod); other than Quarles' bijection, none seems to be known.

(8) Even given a very strong result along the lines of (7), an enormous amount of work would still be needed to obtain applications of non-commutative desingularizations analogous to those of resolutions of singularities. For example, can one define an "arithmetic genus" in a non-commutative context, and show, as Hironaka does, that it is a "birational" invariant?

\section{REFERENCES}

[ADS93] Maurice Auslander, Songqing Ding, and Øyvind Solberg, Liftings and weak liftings of modules, J. Algebra 156 (1993), no. 2, 273-317. MR 1216471 (Cited on page 36). 
[AG60] Maurice Auslander and Oscar Goldman, Maximal orders, Trans. Amer. Math. Soc. 97 (1960), 1-24. MR 0117252 (Cited on page 28).

[AGHZ97] Alexander V. Arhangel'skii, Kenneth R. Goodearl, and Birge Huisgen-Zimmermann, Kiiti Morita (1915-1995), Notices Amer. Math. Soc. 44 (1997), no. 6, 680-684. MR 1452070 (Cited on page 4).

[AM01] Luchezar L. Avramov and Claudia Miller, Frobenius powers of complete intersections, Math. Res. Lett. 8 (2001), no. 1-2, 225-232. MR 1825272 (Cited on page 45).

[AR72] Maurice Auslander and Klaus W. Roggenkamp, A characterization of orders of finite lattice type, Invent. Math. 17 (1972), 79-84. MR 0320053 (Cited on page 38).

[AR89] Maurice Auslander and Idun Reiten, The Cohen-Macaulay type of Cohen-Macaulay rings, Adv. in Math. 73 (1989), no. 1, 1-23. MR 979585 (Cited on page 39).

[ARS97] Maurice Auslander, Idun Reiten, and Sverre O. Smalø, Representation theory of Artin algebras, Cambridge Studies in Advanced Mathematics, vol. 36, Cambridge University Press, Cambridge, 1997, Corrected reprint of the 1995 original. MR 1476671 (Cited on page 5).

[Art97] Michael Artin, Some problems on three-dimensional graded domains, Representation theory and algebraic geometry (Waltham, MA, 1995), London Math. Soc. Lecture Note Ser., vol. 238, Cambridge Univ. Press, Cambridge, 1997, pp. 1-19. MR 1477464 (Cited on page 43).

[AS87] Michael Artin and William F. Schelter, Graded algebras of global dimension 3, Adv. in Math. 66 (1987), no. 2, 171-216. MR 917738 (Cited on page 16).

[AS95] Michael Artin and J. Toby Stafford, Noncommutative graded domains with quadratic growth, Invent. Math. 122 (1995), no. 2, 231-276. MR 1358976 (Cited on page 15).

[Ati58] Michael F. Atiyah, On analytic surfaces with double points, Proc. Roy. Soc. London. Ser. A 247 (1958), 237-244. MR 0095974 (Cited on page 19).

[ATVdB90] Michael Artin, John Tate, and Michel Van den Bergh, Some algebras associated to automorphisms of elliptic curves, The Grothendieck Festschrift, Vol. I, Progr. Math., vol. 86, Birkhäuser Boston, Boston, MA, 1990, pp. 33-85. MR 1086882 (Cited on page 16).

[ATVdB91] _ _ Modules over regular algebras of dimension 3, Invent. Math. 106 (1991), no. 2, $335-388$. MR 1128218 (Cited on page 16).

[Aus62] Maurice Auslander, On the purity of the branch locus, Amer. J. Math. 84 (1962), $116-125$. MR 0137733 (Cited on page 23).

[Aus71] _ Representation dimension of artin algebras, Selected works of Maurice Auslander, vol. 1, 1999, American Mathematical Society, 1971, Originally Queen Mary College Mathematics Notes, University of London, pp. 505-574. (Cited on pages 34 and 37 ).

[Aus78] _ _ Functors and morphisms determined by objects, Representation theory of algebras (Proc. Conf., Temple Univ., Philadelphia, Pa., 1976), Dekker, New York, 1978, pp. 1-244. Lecture Notes in Pure Appl. Math., Vol. 37. MR 0480688 (Cited on page 14).

[Aus86a] _ Isolated singularities and existence of almost split sequences, Representation theory, II (Ottawa, Ont., 1984), Lecture Notes in Math., vol. 1178, Springer, Berlin, 1986, pp. 194-242. MR 842486 (Cited on page 14).

[Aus86b] _ Rational singularities and almost split sequences, Trans. Amer. Math. Soc. 293 (1986), no. 2, 511-531. MR 816307 (Cited on pages 23, 28, and 38).

[AV85] Michael Artin and Jean-Louis Verdier, Reflexive modules over rational double points, Math. Ann. 270 (1985), no. 1, 79-82. MR 769609 (Cited on page 25).

[AVdB90] Michael Artin and Michel Van den Bergh, Twisted homogeneous coordinate rings, J. Algebra 133 (1990), no. 2, 249-271. MR 1067406 (Cited on page 15).

[AZ94] Michael Artin and James J. Zhang, Noncommutative projective schemes, Adv. Math. 109 (1994), no. 2, 228-287. MR 1304753 (Cited on page 15).

[Bae88] Dagmar Baer, Tilting sheaves in representation theory of algebras, Manuscripta Math. 60 (1988), no. 3, 323-347. MR 928291 (Cited on page 10).

[Bal02] Paul Balmer, Presheaves of triangulated categories and reconstruction of schemes, Math. Ann. 324 (2002), no. 3, 557-580. MR 1938458 (Cited on page 9).

[Bal05] _ The spectrum of prime ideals in tensor triangulated categories, J. Reine Angew. Math. 588 (2005), 149-168. MR 2196732 (Cited on page 9). 
[Bas60] Hyman Bass, Finitistic dimension and a homological generalization of semi-primary rings, Trans. Amer. Math. Soc. 95 (1960), 466-488. MR 0157984 (Cited on page 13).

[BB80] Sheila Brenner and Michael C. R. Butler, Generalizations of the Bernstein-Gel'fand-Ponomarev reflection functors, Representation theory, II (Proc. Second Internat. Conf., Carleton Univ., Ottawa, Ont., 1979), Lecture Notes in Math., vol. 832, Springer, Berlin, 1980, pp. 103-169. MR 607151 (Cited on page 8).

[BD02] David Berenstein and Michael R. Douglas, Seiberg duality for quiver gauge theories, http://arxiv.org/abs/hep-th/0207027, 2002. (Cited on page 2).

[BD11] Igor Burban and Yuriy Drozd, Tilting on non-commutative rational projective curves, http://arxiv.org/abs/1103.3936, April 2011. (Cited on page 44).

[Bei08] Charlie Beil, The noncommutative geometry of square superpotential algebras, http://arxiv.org/abs/0811.2439, November 2008. (Cited on page 46).

[Ber02] David Berenstein, Reverse geometric engineering of singularities, J. High Energy Phys. (2002), no. 4, No. 52, 18. MR 1911393 (Cited on page 2).

[Bez06] Roman V. Bezrukavnikov, Noncommutative counterparts of the Springer resolution, International Congress of Mathematicians. Vol. II, Eur. Math. Soc., Zürich, 2006, pp. 1119-1144. MR 2275638 (Cited on pages 21 and 46).

[BGI71] Pierre Berthelot, Alexandere Grothendieck, and Luc Illusie (eds.), Théorie des intersections et théorème de Riemann-Roch, Lecture Notes in Math., no. 225, Springer-Verlag, New York, 1971. MR 0354655 (Cited on page 8).

[BGP73] Joseph N. Bernšteǐn, Izrail' M. Gel'fand, and Vladimir A. Ponomarev, Coxeter functors, and Gabriel's theorem, Uspehi Mat. Nauk 28 (1973), no. 2(170), 19-33. MR 0393065(Cited on page 8).

[BH84] Kenneth A. Brown and Charudatta R. Hajarnavis, Homologically homogeneous rings, Trans. Amer. Math. Soc. 281 (1984), no. 1, 197-208. MR 719665 (Cited on page 28).

[BH93] Winfried Bruns and Jürgen Herzog, Cohen-Macaulay rings, Cambridge Studies in Advanced Mathematics, vol. 39, Cambridge University Press, Cambridge, 1993. MR 1251956 (Cited on pages 17 and 32.

[BHM82] Kenneth A. Brown, Charudatta R. Hajarnavis, and Alistair B. MacEacharn, Noetherian rings of finite global dimension, Proc. London Math. Soc. (3) 44 (1982), no. 2, 349-371. MR 647437 (Cited on pages 13 and 28 .

[BHM83] _ Rings of finite global dimension integral over their centres, Comm. Algebra 11 (1983), no. 1, 67-93. MR 687406 (Cited on page 28).

[BIKR08] Igor Burban, Osamu Iyama, Bernhard Keller, and Idun Reiten, Cluster tilting for onedimensional hypersurface singularities, Adv. Math. 217 (2008), no. 6, 2443-2484. MR 2397457 (Cited on pages 33, 34, 35, and 36).

[BK04] Roman V. Bezrukavnikov and Dmitry B. Kaledin, McKay equivalence for symplectic resolutions of quotient singularities, Tr. Mat. Inst. Steklova 246 (2004), no. Algebr. Geom. Metody, Svyazi i Prilozh., 20-42. MR 2101282 (Cited on page 26).

[BKR01] Tom Bridgeland, Alastair King, and Miles Reid, The McKay correspondence as an equivalence of derived categories, J. Amer. Math. Soc. 14 (2001), no. 3, 535-554 (electronic). MR 1824990 (Cited on pages 2 and 26).

[BKS07] Aslak Bakke Buan, Henning Krause, and Øyvind Solberg, Support varieties: an ideal approach, Homology, Homotopy Appl. 9 (2007), no. 1, 45-74. MR 2280286 (Cited on page 6).

[BL01] David Berenstein and Robert G. Leigh, Resolution of stringy singularities by non-commutative algebras, J. High Energy Phys. (2001), no. 6, Paper 30, 37. MR 1849725 (Cited on pages 2and46).

[BLV10a] Ragnar-Olaf Buchweitz, Graham J. Leuschke, and Michel Van den Bergh, A characteristic free tilting bundle for Grassmannians, http://arxiv.org/abs/1006.1633, June 2010. (Cited on pages 10 and 43.

[BLV10b] _ Non-commutative desingularization of determinantal varieties I, Invent. Math. 182 (2010), no. 1, 47-115. MR 2672281 (Cited on pages 3, 11, 40, 43, and 46).

[BLV11] _ Non-commutative desingularization of determinantal varieties, II: Arbitrary minors, submitted, 2011. (Cited on page 43). 
[BM06] Aslak Bakke Buan and Robert Marsh, Cluster-tilting theory, Trends in representation theory of algebras and related topics, Contemp. Math., vol. 406, Amer. Math. Soc., Providence, RI, 2006, pp. 1-30. MR 2258039 (Cited on page 34).

[BM09] Martin Bender and Sergey Mozgovoy, Crepant resolutions and brane tilings II: Tilting bundles, http://arxiv.org/abs/0909.2013, September 2009. (Cited on page 46).

[B095] Alexei I. Bondal and Dmitri Orlov, Semiorthogonal decompositions for algebraic varieties, http://arxiv.org/abs/alg-geom/9506012, 1995. (Cited on page 46).

[BO01] , Reconstruction of a variety from the derived category and groups of autoequivalences, Compositio Math. 125 (2001), no. 3, 327-344. MR 1818984 (Cited on page9).

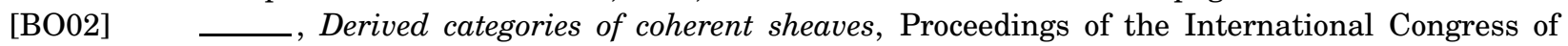
Mathematicians, Vol. II (Beijing, 2002) (Beijing), Higher Ed. Press, 2002, pp. 47-56. MR 1957019 (Cited on pages 2, 20, 21, and 22).

[Boc09] Raf Bocklandt, Calabi-Yau algebras and weighted quiver polyhedra, http://arxiv.org/abs/0905.0232, 2009. (Cited on page 29).

[Bon81] Klaus Bongartz, Tilted algebras, Representations of algebras (Puebla, 1980), Lecture Notes in Math., vol. 903, Springer, Berlin, 1981, pp. 26-38. MR 654701 (Cited on page 8).

[Bon89] Alexei I. Bondal, Representations of associative algebras and coherent sheaves, Izv. Akad. Nauk SSSR Ser. Mat. 53 (1989), no. 1, 25-44. MR 992977 (Cited on page 10).

[BP93] Alexei I. Bondal and Alexander E. Polishchuk, Homological properties of associative algebras: the method of helices, Izv. Ross. Akad. Nauk Ser. Mat. 57 (1993), no. 2, 3-50. MR 1230966 (Cited on page 16.

[Bra07] Amiram Braun, On symmetric, smooth and Calabi-Yau algebras, J. Algebra 317 (2007), no. 2, 519-533. MR 2362929 (Cited on page 29).

[Bri02] Tom Bridgeland, Flops and derived categories, Invent. Math. 147 (2002), no. 3, 613-632. MR 1893007 (Cited on pages 2 and 21).

[Bri06] Derived categories of coherent sheaves, International Congress of Mathematicians. Vol. II, Eur. Math. Soc., Zürich, 2006, pp. 563-582. MR 2275610 (Cited on page 7).

[Bro09] Nathan Broomhead, Dimer models and Calabi-Yau algebras, http://arxiv.org/abs/0901.4662, January 2009. (Cited on page 46).

[BVdB03] Alexei I. Bondal and Michel Van den Bergh, Generators and representability of functors in commutative and noncommutative geometry, Mosc. Math. J. 3 (2003), no. 1, 1-36, 258. MR 1996800 (Cited on pages 5, 9, 10, 30, and 34).

[Căl05] Andrei Căldăraru, Derived categories of sheaves: a skimming, Snowbird lectures in algebraic geometry, Contemp. Math., vol. 388, Amer. Math. Soc., Providence, RI, 2005, pp. 43-75. MR 2182889 (Cited on page 8).

[CBH98] William W. Crawley-Boevey and Martin P. Holland, Noncommutative deformations of Kleinian singularities, Duke Math. J. 92 (1998), no. 3, 605-635. MR 1620538 (Cited on page 45.

[CL09] Sabin Cautis and Timothy Logvinenko, A derived approach to geometric McKay correspondence in dimension three, J. Reine Angew. Math. 636 (2009), 193-236. MR 2572250 (Cited on page 26).

[Cor04] Alessio Corti, What is...a flip?, Notices Amer. Math. Soc. 51 (2004), no. 11, 1350-1351. MR 2105240 (Cited on page 19).

[CPS86] Edward T. Cline, Brian J. Parshall, and Leonard L. Scott, Derived categories and Morita theory, J. Algebra 104 (1986), no. 2, 397-409. MR 866784 (Cited on page 8).

[Dao08] Hailong Dao, Some observations on local and projective hypersurfaces, Math. Res. Lett. 15 (2008), no. 2, 207-219. MR 2385635 (Cited on page 33).

[Dao10] _ Remarks on non-commutative crepant resolutions of complete intersections, Adv. Math. 224 (2010), no. 3, 1021-1030. MR 2628801 (Cited on pages 32, 33, and 45).

[Dao11] _ Decent intersection and Tor-rigidity for modules over local hypersurfaces, to appear in Trans. AMS, http://arxiv.org/abs/math/0611568, February 2011. (Cited on page 33).

[DdJGP99] Wolfram Decker, Theo de Jong, Gert-Martin Greuel, and Gerhard Pfister, The normalization: a new algorithm, implementation and comparisons, Computational methods for representations of groups and algebras (Essen, 1997), Progr. Math., vol. 173, Birkhäuser, Basel, 1999, pp. 177-185. MR 1714609 (Cited on page 31). 
[DGM97] Michael R. Douglas, Brian R. Greene, and David R. Morrison, Orbifold resolution by D-branes, Nucl. Phys. B506 (1997), 84-106. (Cited on page 2).

[DH10] Hailong Dao and Craig Huneke, Vanishing of Ext, cluster tilting modules and finite global dimension of endomorphism rings, http://arxiv.org/abs/1005.5359, May 2010. (Cited on page 35).

[DHVW85] Lance J. Dixon, Jeffrey A. Harvey, Cumrun Vafa, and Edward Witten, Strings on orbifolds, Nuclear Phys. B 261 (1985), no. 4, 678-686, DOI MR 818423 (Cited on page 25).

[dJ98] Theo de Jong, An algorithm for computing the integral closure, J. Symbolic Comput. 26 (1998), no. 3, 273-277. MR 1633931 (Cited on page 31).

[Eis80] David Eisenbud, Homological algebra on a complete intersection, with an application to group representations, Trans. Amer. Math. Soc. 260 (1980), no. 1, 35-64. MR 570778 (Cited on page 40).

[Eis95] , Commutative algebra, Graduate Texts in Mathematics, vol. 150, Springer-Verlag, New York, 1995, With a view toward algebraic geometry. MR 1322960 (Cited on pages 11 and 42).

[Esn85] Hélène Esnault, Reflexive modules on quotient surface singularities, J. Reine Angew. Math. 362 (1985), 63-71. MR 809966 (Cited on pages 25 and 38).

[ESW03] David Eisenbud, Frank-Olaf Schreyer, and Jerzy Weyman, Resultants and Chow forms via exterior syzygies, J. Amer. Math. Soc. 16 (2003), no. 3, 537-579 (electronic). MR 1969204 (Cited on page 43.

[Fre64] Peter Freyd, Abelian categories. An introduction to the theory of functors, Harper's Series in Modern Mathematics, Harper \& Row Publishers, New York, 1964. MR 0166240 (Cited on page5).

[Gab62] Pierre Gabriel, Des catégories abéliennes, Bull. Soc. Math. France 90 (1962), 323-448. MR 0232821 (Cited on pages 4 and 6).

[Gin05] Victor Ginzburg, Lectures on Noncommutative Geometry, http://arXiv.org/abs/0506603, June 2005. (Cited on page 12).

[Gin06] Calabi-Yau algebras, http://arXiv.org/abs//0612139, December 2006. (Cited on page 29].

[GL87] Werner Geigle and Helmut Lenzing, A class of weighted projective curves arising in representation theory of finite-dimensional algebras, Singularities, representation of algebras, and vector bundles (Lambrecht, 1985), Lecture Notes in Math., vol. 1273, Springer, Berlin, 1987, pp. 265297. MR 915180 (Cited on pages 10 and 15).

[GLS10] Gert-Martin Greuel, Santiago Laplagne, and Frank Seelisch, Normalization of rings, J. Symbolic Comput. 45 (2010), no. 9, 887-901. MR 2661161 (Cited on page 31).

[Goo89] Kenneth R. Goodearl, Global dimension of differential operator rings. IV. Simple modules, Forum Math. 1 (1989), no. 2, 185-200. MR 990143 (Cited on page 14).

[GR70] Hans Grauert and Oswald Riemenschneider, Verschwindungssätze für analytische Kohomologiegruppen auf komplexen Räumen, Invent. Math. 11 (1970), 263-292. MR 0302938 (Cited on pages [16 and 47).

[GR71] Hans Grauert and Reinhold Remmert, Analytische Stellenalgebren, Springer-Verlag, Berlin, 1971, Unter Mitarbeit von O. Riemenschneider, Die Grundlehren der mathematischen Wissenschaften, Band 176. MR 0316742 (Cited on page 31).

[GR84] Coherent analytic sheaves, Grundlehren der Mathematischen Wissenschaften [Fundamental Principles of Mathematical Sciences], vol. 265, Springer-Verlag, Berlin, 1984. MR 755331 (Cited on page 31).

[Gro61] Alexandre Grothendieck, Éléments de géométrie algébrique. III. Étude cohomologique des faisceaux cohérents. I, Inst. Hautes Études Sci. Publ. Math. (1961), no. 11, 167. MR 0217085 (Cited on page 10).

[GS] Daniel Grayson and Michael Stillman, Macaulay 2, a software system for research in algebraic geometry, Available at http://www .math.uiuc.edu/Macaulay2/. (Cited on page 39).

[GS04] Iain Gordon and S. Paul Smith, Representations of symplectic reflection algebras and resolutions of deformations of symplectic quotient singularities, Math. Ann. 330 (2004), no. 1, 185-200. MR 2091684 (Cited on page 45).

[GSV81] Gerardo González-Sprinberg and Jean-Louis Verdier, Points doubles rationnels et représentations de groupes, C. R. Acad. Sci. Paris Sér. I Math. 293 (1981), no. 2, 111-113. MR 637103 (Cited on page 25. 
[Hap87] Dieter Happel, On the derived category of a finite-dimensional algebra, Comment. Math. Helv. 62 (1987), no. 3, 339-389. MR 910167 (Cited on page 7).

[Hap88] _ Triangulated categories in the representation theory of finite-dimensional algebras, London Mathematical Society Lecture Note Series, vol. 119, Cambridge University Press, Cambridge, 1988. MR 935124 (Cited on pages 7 and 8).

[Hap89] _ Hochschild cohomology of finite-dimensional algebras, Séminaire d'Algèbre Paul Dubreil et Marie-Paul Malliavin, 39ème Année (Paris, 1987/1988), Lecture Notes in Math., vol. 1404, Springer, Berlin, 1989, pp. 108-126. MR 1035222 (Cited on page 7).

[Har77] Robin Hartshorne, Algebraic geometry, Springer-Verlag, New York, 1977, Graduate Texts in Mathematics, No. 52. MR 0463157 (Cited on pages 9, 11, 13, 16, 18, and 41).

[HE71] Melvin Hochster and John A. Eagon, Cohen-Macaulay rings, invariant theory, and the generic perfection of determinantal loci, Amer. J. Math. 93 (1971), 1020-1058. MR 0302643 (Cited on page 23.

[Her78] Jürgen Herzog, Ringe mit nur endlich vielen Isomorphieklassen von maximalen, unzerlegbaren Cohen-Macaulay-Moduln, Math. Ann. 233 (1978), no. 1, 21-34. MR 0463155 (Cited on page 24).

[HJ10] Thorsten Holm and Peter Jørgensen, Triangulated categories: definitions, properties, and examples, Triangulated categories, London Math. Soc. Lecture Note Ser., vol. 375, Cambridge Univ. Press, Cambridge, 2010, pp. 1-51. MR 2681706 (Not cited).

[HK71] Jürgen Herzog and Ernst Kunz (eds.), Der kanonische Modul eines Cohen-Macaulay-Rings, Lecture Notes in Mathematics, Vol. 238, Springer-Verlag, Berlin, 1971, Seminar über die lokale Kohomologietheorie von Grothendieck, Universität Regensburg, Wintersemester 1970/1971. MR 0412177 (Cited on page 16).

[HM99] Sam Huckaba and Thomas Marley, On associated graded rings of normal ideals, J. Algebra 222 (1999), no. 1, 146-163. MR 1728165 (Cited on page 47).

[Hoc73] Melvin Hochster, Cohen-Macaulay modules, Conference on Commutative Algebra (Univ. Kansas, Lawrence, Kan., 1972), Springer, Berlin, 1973, pp. 120-152. Lecture Notes in Math., Vol. 311. MR 0340251 (Cited on page 32).

[HP] Lutz Hille and Markus Perling, Exceptional sequences of invertible sheaves on rational surfaces, http://arXiv.org/abs/0810.1936. (Cited on page 10).

[HP06] , A counterexample to King's conjecture, Compos. Math. 142 (2006), no. 6, 1507-1521. MR 2278758 (Cited on page 10).

[HR82] Dieter Happel and Claus Michael Ringel, Tilted algebras, Trans. Amer. Math. Soc. 274 (1982), no. 2, 399-443. MR 675063 (Cited on page 8).

[Hun96] Craig Huneke, Tight closure and its applications, CBMS Regional Conference Series in Mathematics, vol. 88, Published for the Conference Board of the Mathematical Sciences, Washington, DC, 1996, With an appendix by Melvin Hochster. MR 1377268 (Cited on pages 17]and 47).

[Huy06] Daniel Huybrechts, Fourier-Mukai transforms in algebraic geometry, Oxford Mathematical Monographs, The Clarendon Press Oxford University Press, Oxford, 2006. MR 2244106 (Cited on pages 8 and 46 .

[HVdB07] Lutz Hille and Michel Van den Bergh, Fourier-Mukai transforms, Handbook of tilting theory, London Math. Soc. Lecture Note Ser., vol. 332, Cambridge Univ. Press, Cambridge, 2007, pp. 147177. MR 2384610 (Cited on pages 8] and 10).

[HZ95] Birge Huisgen-Zimmermann, The finitistic dimension conjectures—a tale of 3.5 decades, Abelian groups and modules (Padova, 1994), Math. Appl., vol. 343, Kluwer Acad. Publ., Dordrecht, 1995, pp. 501-517. MR 1378224 (Cited on page 13).

[IN99] Yukari Ito and Iku Nakamura, Hilbert schemes and simple singularities, New trends in algebraic geometry (Warwick, 1996), London Math. Soc. Lecture Note Ser., vol. 264, Cambridge Univ. Press, Cambridge, 1999, pp. 151-233. MR 1714824 (Cited on page 25).

[IR96] Yukari Ito and Miles Reid, The McKay correspondence for finite subgroups of SL(3,C), Higher-dimensional complex varieties (Trento, 1994), de Gruyter, Berlin, 1996, pp. 221-240. MR 1463181 (Cited on page 18). 
[IR08] Osamu Iyama and Idun Reiten, Fomin-Zelevinsky mutation and tilting modules over Calabi-Yau algebras, Amer. J. Math. 130 (2008), no. 4, 1087-1149. MR 2427009 (Cited on pages 13, 14, 28, 29, and 30].

[Ito95a] Yukari Ito, Crepant resolution of trihedral singularities and the orbifold Euler characteristic, Internat. J. Math. 6 (1995), no. 1, 33-43. MR 1307302 (Cited on page 18).

[Ito95b] _ Gorenstein quotient singularities of monomial type in dimension three, J. Math. Sci. Univ. Tokyo 2 (1995), no. 2, 419-440. MR 1366564 (Cited on page 18).

[IW10] Osamu Iyama and Michael Wemyss, Auslander-Reiten duality and maximal modifications for non-isolated singularities, Math. Z. 265 (2010), no. 1, 41-83. MR 2606949 (Cited on pages 14 and 47.

[IW11] January 2011. (Cited on pages 31 and 46).

[Iya03] Osamu Iyama, Finiteness of representation dimension, Proc. Amer. Math. Soc. 131 (2003), no. 4, 1011-1014 (electronic). MR 1948089 (Cited on page 34).

[Iya07a] _ _ Auslander correspondence, Adv. Math. 210 (2007), no. 1, 51-82. MR 2298820 (Cited on pages 35 and 38.

[Iya07b] , Higher-dimensional Auslander-Reiten theory on maximal orthogonal subcategories, Adv. Math. 210 (2007), no. 1, 22-50. MR 2298819 (Cited on pages 32 and 34).

[Jat86] Arun Vinayak Jategaonkar, Localization in Noetherian rings, London Mathematical Society Lecture Note Series, vol. 98, Cambridge University Press, Cambridge, 1986. MR 839644 (Cited on page 13).

[Jin75] M. I. Jinnah, Reflexive modules over regular local rings, Arch. Math. (Basel) 26 (1975), no. 4, 367-371. MR 0376662 (Cited on page 32).

[JM97] Vaughan Jones and Henri Moscovici, Review of Noncommutative geometry [Academic Press, San Diego, CA, 1994; 1303779 (95j:46063)] by Alain Connes, Notices Amer. Math. Soc. 44 (1997), no. 7, 792-799. MR 1460207 (Cited on page 13).

[Jør] Peter Jørgensen, Non-commutative projective geometry, lecture notes, available from http://www.staff.ncl.ac.uk/peter.jorgensen/manus/opgteach.ps (Cited on page 12).

[Jor08] David A. Jorgensen, Finite projective dimension and the vanishing of $\operatorname{Ext}_{R}(M, M)$, Comm. Algebra 36 (2008), no. 12, 4461-4471. MR 2473341 (Cited on page 33).

[Jot75] P. Jothilingam, A note on grade, Nagoya Math. J. 59 (1975), 149-152. MR 0392979 (Cited on page 33.

[Kal08] Dmitry B. Kaledin, Lecture notes for the course "Homological methods in Non-commutative Geometry", Univ. of Tokyo, Oct. 2007-Mar. 2008, http://imperium.lenin.ru/ kaledin/tokyo/, 2008. (Cited on page 12).

[Kal09] _ Lecture notes for the mini-course "Non-commutative Geometry from the homological point of view", KIAS, Seoul, Oct. 2009, http://imperium.lenin.ru/ kaledin/seoul/, 2009. (Cited on page 12).

[Kap84] Mikhail M. Kapranov, Derived category of coherent sheaves on Grassmann manifolds, Izv. Akad. Nauk SSSR Ser. Mat. 48 (1984), no. 1, 192-202. MR 733363 (Cited on page 10).

[Kap86] Derived category of coherent bundles on a quadric, Funktsional. Anal. i Prilozhen. 20 (1986), no. 2, 67. MR 847146 (Cited on page 10).

[Kap88] _ On the derived categories of coherent sheaves on some homogeneous spaces, Invent. Math. 92 (1988), no. 3, 479-508. MR 939472 (Cited on page 10).

[Kaw06] Yujiro Kawamata, Derived categories of toric varieties, Michigan Math. J. 54 (2006), no. 3, 517535. MR 2280493 (Cited on page 26).

[Kee03] Dennis S. Keeler, The rings of noncommutative projective geometry, Advances in algebra and geometry (Hyderabad, 2001), Hindustan Book Agency, New Delhi, 2003, pp. 195-207. MR 1988956 (Cited on page 12).

[Kin97] Alistair King, Tilting bundles on some rational surfaces, unpublished, http://www.maths.bath.ac.uk/ masadk/papers/, 1997. (Cited on page 10). 
[KKMSD73] George Kempf, Finn Faye Knudsen, David Mumford, and Bernard Saint-Donat, Toroidal embeddings. I, Lecture Notes in Mathematics, Vol. 339, Springer-Verlag, Berlin, 1973. MR 0335518 (Cited on page 17).

[Kle93] Felix Klein, Vorlesungen über das Ikosaeder und die Auflösung der Gleichungen vom fünften Grade (Lectures on the icosahedron and the solution of the 5th degree equations), Basel: Birkhäuser Verlag. Stuttgart: B. G. Teubner Verlagsgesellschaft. xxviii, 343 S. , 1993 (German). (Cited on page 24).

[KM98] János Kollár and Shigefumi Mori, Birational geometry of algebraic varieties, Cambridge Tracts in Mathematics, vol. 134, Cambridge University Press, Cambridge, 1998, With the collaboration of C. H. Clemens and A. Corti, Translated from the 1998 Japanese original. MR 1658959 (Cited on page 20).

[Knö85] Horst Knörrer, Group representations and the resolution of rational double points, Finite groups-coming of age (Montreal, Que., 1982), Contemp. Math., vol. 45, Amer. Math. Soc., Providence, RI, 1985, pp. 175-222. MR 822239 (Cited on page 25).

[Kol89a] János Kollár, Flops, Nagoya Math. J. 113 (1989), 15-36. MR 986434 (Cited on page 20).

[Kol89b] _ Minimal models of algebraic threefolds: Mori's program, Astérisque (1989), no. 177-178, Exp. No. 712, 303-326, Séminaire Bourbaki, Vol. 1988/89. MR 1040578 (Cited on page 19).

[Kol07] What is ... a minimal model?, Notices Amer. Math. Soc. 54 (2007), no. 3, 370-371. MR 2292143 (Cited on page 19).

[Kon95] Maxim Kontsevich, Homological algebra of mirror symmetry, Proceedings of the International Congress of Mathematicians, Vol. 1, 2 (Zürich, 1994) (Basel), Birkhäuser, 1995, pp. 120-139. MR 1403918 (Cited on page 7).

[KS09] Maxim Kontsevich and Yan Soibelman, Notes on $A_{\infty}$-algebras, $A_{\infty}$-categories and noncommutative geometry, Homological mirror symmetry, Lecture Notes in Phys., vol. 757, Springer, Berlin, 2009, pp. 153-219. MR 2596638 (Cited on page 22).

[Kuz08] Alexander Kuznetsov, Lefschetz decompositions and categorical resolutions of singularities, Selecta Math. (N.S.) 13 (2008), no. 4, 661-696. MR 2403307 (Cited on pages 21, 22, and 43).

[KV00] Mikhail M. Kapranov and Eric Vasserot, Kleinian singularities, derived categories and Hall algebras, Math. Ann. 316 (2000), no. 3, 565-576. MR 1752785 (Cited on page 26).

[Lam99] T. Y. Lam, Lectures on modules and rings, Graduate Texts in Mathematics, vol. 189, SpringerVerlag, New York, 1999. MR 1653294 (Cited on page 5).

[Lau03] Olav A. Laudal, Noncommutative algebraic geometry, Proceedings of the International Conference on Algebraic Geometry and Singularities (Spanish) (Sevilla, 2001), vol. 19, 2003, pp. 509580. MR 2023198 (Cited on page 12).

[Leu07] Graham J. Leuschke, Endomorphism rings of finite global dimension, Canad. J. Math. 59 (2007), no. 2, 332-342. MR 2310620 (Cited on pages 38 and 39).

[Lin02] Hui-Wen Lin, On crepant resolution of some hypersurface singularities and a criterion for UFD, Trans. Amer. Math. Soc. 354 (2002), no. 5, 1861-1868 (electronic). MR 1881020 (Cited on page 17).

[Lun10] Valery A. Lunts, Categorical resolution of singularities, J. Algebra 323 (2010), no. 10, 2977-3003. MR 2609187 (Cited on pages 21] and 22).

[LW] Graham J. Leuschke and Roger Wiegand, Cohen-Macaulay Representations, in progress, available from http://www. leuschke.org/Research/MCMBook. (Cited on page 23).

[Mac10] Marjory Jane Macleod, Generalizing the Cohen-Macaulay condition and other homological properities, Ph.D. thesis, University of Glasgow, 2010, http://theses .gla.ac.uk/2004/, p. 144 pages. (Cited on page 37).

[Mar97] Dimitri Markushevich, Resolution of $\mathbb{C}^{3} / H_{168}$, Math. Ann. 308 (1997), no. 2, 279-289. MR 1464903 (Cited on page 18).

[McK80] John McKay, Graphs, singularities, and finite groups, The Santa Cruz Conference on Finite Groups (Univ. California, Santa Cruz, Calif., 1979), Proc. Sympos. Pure Math., vol. 37, Amer. Math. Soc., Providence, R.I., 1980, pp. 183-186. MR 604577 (Cited on pages 23, 24, and 25).

[Miy86] Yoichi Miyashita, Tilting modules of finite projective dimension, Math. Z. 193 (1986), no. 1, 113146. MR 852914 (Cited on page 8). 
[ML98] Saunders Mac Lane, Categories for the working mathematician, second ed., Graduate Texts in Mathematics, vol. 5, Springer-Verlag, New York, 1998. MR 1712872 (Cited on page 4).

[MOa] Existence of non-commutative desingularizations, MathOverflow, URL: http://mathoverflow.net/questions/14350 (version: 2010-02-06). (Cited on page 37).

[MOb] Flips in the minimal model program, MathOverflow, URL: http://mathoverflow.net/questions/5321 (version: 2009-11-16). (Cited on page 19).

[MOc] Non-commutative algebraic geometry, MathOverflow, URL:http://mathoverflow.net/questions/7917 (version: 2009-12-14). (Cited on page 13).

[Mor58] Kiiti Morita, Duality for modules and its applications to the theory of rings with minimum condition, Sci. Rep. Tokyo Kyoiku Daigaku Sect. A 6 (1958), 83-142. MR 0096700 (Cited on page 4).

[Mor08] Izuru Mori, An introduction to noncommutative algebraic geometry, Proceedings of the 40th Symposium on Ring Theory and Representation Theory, Symp. Ring Theory Represent. Theory Organ. Comm., Yamaguchi, 2008, pp. 53-59. MR 2427915 (Cited on page 12).

[Moz09] Sergey Mozgovoy, Crepant resolutions and brane tilings I: Toric realization, http://arxiv. org/abs/0908.3475, August 2009. (Cited on page 46).

[Muk81] Shigeru Mukai, Duality between $D(X)$ and $D(\hat{X})$ with its application to Picard sheaves, Nagoya Math. J. 81 (1981), 153-175. MR 607081 (Cited on page 8).

[Nak01] Iku Nakamura, Hilbert schemes of abelian group orbits, J. Algebraic Geom. 10 (2001), no. 4, 757-779. MR 1838978 (Cited on page 25).

[Or197] Dmitri O. Orlov, Equivalences of derived categories and K3 surfaces, J. Math. Sci. (New York) 84 (1997), no. 5, 1361-1381, Algebraic geometry, 7. MR 1465519 (Cited on page 8).

[Orl03a] Derived categories of coherent sheaves and equivalences between them, Uspekhi Mat. Nauk 58 (2003), no. 3(351), 89-172. MR 1998775(Not cited).

[Orl03b] Quasicoherent sheaves in commutative and noncommutative geometry, Izv. Ross. Akad. Nauk Ser. Mat. 67 (2003), no. 3, 119-138. MR 1992195 (Not cited).

[Orl09a] Derived categories of coherent sheaves and triangulated categories of singularities, Algebra, arithmetic, and geometry: in honor of Yu. I. Manin. Vol. II, Progr. Math., vol. 270, Birkhäuser Boston Inc., Boston, MA, 2009, pp. 503-531. MR 2641200(Not cited).

[Orl09b] _ Remarks on generators and dimensions of triangulated categories, Mosc. Math. J. 9 (2009), no. 1, 153-159, back matter. MR 2567400 (Not cited).

[OY09] Hirosi Ooguri and Masahito Yamazaki, Crystal melting and toric Calabi-Yau manifolds, Comm. Math. Phys. 292 (2009), no. 1, 179-199. MR 2540074 (Cited on page 46).

[Pas89] Donald S. Passman, Infinite crossed products, Pure and Applied Mathematics, vol. 135, Academic Press Inc., Boston, MA, 1989. MR 979094 (Cited on page 13).

[Qua05] Christopher L. Quarles, Krull-Schmidt Rings \& Noncommutative Resolutions of Singularities, Master's thesis, University of Washingon, 2005. (Cited on pages 38 and 44).

[Rai87] John Rainwater, Global dimension of fully bounded Noetherian rings, Comm. Algebra 15 (1987), no. 10, 2143-2156. MR 909958 (Cited on page 14).

[Ram69] Mark Ramras, Maximal orders over regular local rings of dimension two, Trans. Amer. Math. Soc. 142 (1969), 457-479. MR 0245572 (Cited on page 14).

[Ran06] Andrew Ranicki (ed.), Non-commutative localization in algebra and topology, London Mathematical Society Lecture Note Series, vol. 330, Cambridge, Cambridge University Press, 2006. MR 2222649 (Cited on page 13).

[Rei83] Miles Reid, Minimal models of canonical 3-folds, Algebraic varieties and analytic varieties (Tokyo, 1981), Adv. Stud. Pure Math., vol. 1, North-Holland, Amsterdam, 1983, pp. 131-180. MR 715649 (Cited on page 19).

[Rei00] Twenty-five years of 3-folds—an old person's view, Explicit birational geometry of 3-folds, London Math. Soc. Lecture Note Ser., vol. 281, Cambridge Univ. Press, Cambridge, 2000, pp. 313343. MR 1798985 (Cited on pages 2 and 17).

[Rei02] La correspondance de McKay, Astérisque (2002), no. 276, 53-72, Séminaire Bourbaki, Vol. 1999/2000. MR 1886756 (Cited on pages 18 and 26). 
[Rei03] Irving Reiner, Maximal orders, London Mathematical Society Monographs. New Series, vol. 28, The Clarendon Press Oxford University Press, Oxford, 2003, Corrected reprint of the 1975 original, With a foreword by M. J. Taylor. MR 1972204 (Cited on page 28).

[Ric89] Jeremy Rickard, Morita theory for derived categories, J. London Math. Soc. (2) 39 (1989), no. 3, 436-456. MR 1002456 (Cited on pages 7]and 8).

[Ric91] _ _ Derived equivalences as derived functors, J. London Math. Soc. (2) 43 (1991), no. 1, 37-48. MR 1099084 (Cited on page 7).

[Roa94] Shi-Shyr Roan, On $c_{1}=0$ resolution of quotient singularity, Internat. J. Math. 5 (1994), no. 4, 523-536. MR 1284568 (Cited on page 18).

[Roa96] _ Minimal resolutions of Gorenstein orbifolds in dimension three, Topology 35 (1996), no. 2, 489-508. MR 1380512 (Cited on page 18).

[Ros98a] Alexander L. Rosenberg, Noncommutative schemes, Compositio Math. 112 (1998), no. 1, 93-125. MR 1622759 (Cited on page 6).

[Ros98b] _ The spectrum of abelian categories and reconstruction of schemes, Rings, Hopf algebras, and Brauer groups (Antwerp/Brussels, 1996), Lecture Notes in Pure and Appl. Math., vol. 197, Dekker, New York, 1998, pp. 257-274. MR 1615928 (Cited on page 6).

[Rou06a] Raphaël Rouquier, Catégories dérivées et géométrie birationnelle (d'après Bondal, Orlov, Bridgeland, Kawamata et al.), Astérisque (2006), no. 307, Exp. No. 946, viii, 283-307, Séminaire Bourbaki. Vol. 2004/2005. MR 2296422 (Not cited).

[Rou06b] _ Representation dimension of exterior algebras, Invent. Math. 165 (2006), no. 2, 357-367. MR 2231960 (Cited on page 34).

[Rou08] _ _ Dimensions of triangulated categories, J. K-Theory 1 (2008), no. 2, 193-256. MR 2434186 (Cited on page 34).

[Rud90] Alexei N. Rudakov, Exceptional collections, mutations and helices, Helices and vector bundles, London Math. Soc. Lecture Note Ser., vol. 148, Cambridge Univ. Press, Cambridge, 1990, pp. 16. MR 1074777 (Cited on page 46).

[SdS87] Juan B. Sancho de Salas, Blowing-up morphisms with Cohen-Macaulay associated graded rings, Géométrie algébrique et applications, I (La Rábida, 1984), Travaux en Cours, vol. 22, Hermann, Paris, 1987, pp. 201-209. MR 907914 (Cited on page 47).

[Ser55] Jean-Pierre Serre, Faisceaux algébriques cohérents, Ann. of Math. (2) 61 (1955), $197-278$. MR 0068874 (Cited on page 6).

[Smi] S. Paul Smith, Non-commutative algebraic geometry, draft of 2000, http://www.math. washington.edu/ smith/Research/nag.pdf. (Cited on page 12).

[SQ05] S. Paul Smith and Christopher Quarles, On an example of Leuschke, private communication, 2005. (Cited on page 39).

[SVdB97] Karen E. Smith and Michel Van den Bergh, Simplicity of rings of differential operators in prime characteristic, Proc. London Math. Soc. (3) 75 (1997), no. 1, 32-62. MR 1444312 (Cited on page 45).

[SVdB01] J. Toby Stafford and Michel Van den Bergh, Noncommutative curves and noncommutative surfaces, Bull. Amer. Math. Soc. (N.S.) 38 (2001), no. 2, 171-216. MR 1816070 (Cited on pages 12 and 15.

[SVdB08] _ Noncommutative resolutions and rational singularities, Michigan Math. J. 57 (2008), 659-674, Special volume in honor of Melvin Hochster. MR 2492474 (Cited on pages [36, 37, and 47).

[SW99] Nathan Seiberg and Edward Witten, String theory and noncommutative geometry, J. High Energy Phys. (1999), no. 9, Paper 32, 93 pp. (electronic). MR 1720697(Cited on page 46).

[Tod08a] Yukinobu Toda, Birational Calabi-Yau threefolds and BPS state counting, Commun. Number Theory Phys. 2 (2008), no. 1, 63-112. MR 2417847(Cited on page 44).

[Tod08b] _ Stability conditions and crepant small resolutions, Trans. Amer. Math. Soc. 360 (2008), no. 11, 6149-6178. MR 2425708 (Cited on page 44).

[Tod09] _ Stability conditions and Calabi-Yau fibrations, J. Algebraic Geom. 18 (2009), no. 1, 101133. MR 2448280 (Cited on page 44). 
[TV08] Bertrand Toën and Michel Vaquié, Algébrisation des variétés analytiques complexes et catégories dérivées, Math. Ann. 342 (2008), no. 4, 789-831. MR 2443764 (Cited on page 22).

[TY09] Yukinobu Toda and Takehiko Yasuda, Noncommutative resolution, $F$-blowups and D-modules, Adv. Math. 222 (2009), no. 1, 318-330. MR 2531377 (Cited on pages 44 and 45).

[Vas68] Wolmer V. Vasconcelos, Reflexive modules over Gorenstein rings, Proc. Amer. Math. Soc. 19 (1968), 1349-1355. MR 0237480 (Cited on page 32).

[Vas73] _ On quasi-local regular algebras, Symposia Mathematica, Vol. XI (Convegno di Algebra Commutativa, INDAM, Rome, 1971), Academic Press, London, 1973, pp. 11-22. MR 0330159 (Cited on page 28).

[VdB87] Michel Van den Bergh, Regular rings of dimension three, Séminaire d'algèbre Paul Dubreil et Marie-Paule Malliavin (Paris, 1986), Lecture Notes in Math., vol. 1296, Springer, Berlin, 1987, pp. 228-234. MR 932058 (Cited on page 16).

[VdB01] _ Blowing up of non-commutative smooth surfaces, Mem. Amer. Math. Soc. 154 (2001), no. 734, $\mathrm{x}+140$. MR 1846352 (Cited on pages 15, 43, and 44).

[VdB04a] , Non-commutative crepant resolutions (with some corrections), The legacy of Niels Henrik Abel, Springer, Berlin, 2004, (the updated [2009] version on ArXiV has some minor corrections over the published version), pp. 749-770. [MR 2077594 (Cited on pages 28, 30, 36, and 47).

[VdB04b] - Three-dimensional flops and noncommutative rings, Duke Math. J. 122 (2004), no. 3, 423-455. MR 2057015 (Cited on pages 2, 27, 28, and 30).

[Ver92a] Andrey B. Verëvkin, Injective Serre sheaves, Mat. Zametki 52 (1992), no. 4, 35-41, 128. MR 1203950 (Cited on page 15).

[Ver92b] On a noncommutative analogue of the category of coherent sheaves on a projective scheme, Algebra and analysis (Tomsk, 1989), Amer. Math. Soc. Transl. Ser. 2, vol. 151, Amer. Math. Soc., Providence, RI, 1992, pp. 41-53. MR 1191171 (Cited on page 15).

[Ver96] Jean-Louis Verdier, Des catégories dérivées des catégories abéliennes, Astérisque (1996), no. 239, xii+253 pp. (1997), With a preface by Luc Illusie, Edited and with a note by Georges Maltsiniotis. MR 1453167 (Cited on page 6).

[Vie77] Eckart Viehweg, Rational singularities of higher dimensional schemes, Proc. Amer. Math. Soc. 63 (1977), no. 1, 6-8. MR 0432637 (Cited on page 17).

[VW97] Alain Verschoren and Luc Willaert, Noncommutative algebraic geometry: from pi-algebras to quantum groups, Bull. Belg. Math. Soc. Simon Stevin 4 (1997), no. 5, 557-588. MR 1600288 (Cited on page 12).

[Wat74] Keiichi Watanabe, Certain invariant subrings are Gorenstein. I, II, Osaka J. Math. 11 (1974), 1-8; ibid. 11 (1974), 379-388. MR 0354646 (Cited on pages 17)and 24).

[Wem07] Michael Wemyss, Reconstruction Algebras of Type A, http://arXiv.org/abs/0704.3693, April 2007. (Cited on page 46).

[Wem09a] Reconstruction Algebras of Type D (I), http://arXiv.org/abs/0905.1154. May 2009. (Cited on page 46).

[Wem09b] , Reconstruction Algebras of Type D (II), http://arXiv.org/abs/0905.1155, May 2009. (Cited on page 46).

[Wey03] Jerzy Weyman, Cohomology of vector bundles and syzygies, Cambridge Tracts in Mathematics, vol. 149, Cambridge University Press, Cambridge, 2003. MR 1988690 (Cited on page 43).

[Yas09] Takehiko Yasuda, Frobenius morphisms of noncommutative blowups, http://arXiv.org/abs/0906.1662, June 2009. (Cited on pages 44and45).

[Yek92] Amnon Yekutieli, Dualizing complexes over noncommutative graded algebras, J. Algebra 153 (1992), no. 1, 41-84. MR 1195406 (Cited on page 37).

[Yos90] Yuji Yoshino, Cohen-Macaulay modules over Cohen-Macaulay rings, London Mathematical Society Lecture Note Series, vol. 146, Cambridge University Press, Cambridge, 1990. MR 1079937 (Cited on pages 23 and 34).

Mathematics Department, Syracuse University, Syracuse NY 13244, USA

E-mail address: gjleusch@math.syr.edu

$U R L$ : www .leuschke.org 